\title{
Fifty Years of Noise Modeling and Mitigation in Power-Line Communications
}

\author{
Tong Bai, Hongming Zhang, Jingjing Wang, Chao Xu, Maged Elkashlan, \\ Arumugam Nallanathan, Fellow, IEEE, and Lajos Hanzo, Fellow, IEEE
}

\begin{abstract}
Building on the ubiquity of electric power infrastructure, power line communications (PLC) has been successfully used in diverse application scenarios, including the smart grid and in-home broadband communications systems as well as industrial and home automation. However, the power line channel exhibits deleterious properties, one of which is its hostile noise environment. This article aims for providing a review of noise modeling and mitigation techniques in PLC. Specifically, a comprehensive review of representative noise models developed over the past fifty years is presented, including both the empirical models based on measurement campaigns and simplified mathematical models. Following this, we provide an extensive survey of the suite of noise mitigation schemes, categorizing them into mitigation at the transmitter as well as parametric and nonparametric techniques employed at the receiver. Furthermore, since the accuracy of channel estimation in PLC is affected by noise, we review the literature of joint noise mitigation and channel estimation solutions. Finally, a number of directions are outlined for future research on both noise modeling and mitigation in PLC.
\end{abstract}

Index Terms-Power-line communications, background noise, impulsive noise, narrow-band interference, noise modeling and mitigation techniques.

\section{INTRODUCTION}

\section{A. Power-Line Communications}

Power-line communications (PLC) has been considered as a means of data transmission since the late 19th century [1, Ch. 1]. Early applications include narrow-band voice and data communications over both medium- and high-voltage power lines for telemetry, telecontrol and teleprotection purposes, as the predecessors of to contemporary smart grid communications [2]-[6]. However, communications over power-lines have not received wider attention until the late 1990s [7], when both the telecommunications services and the electricity industry

T. Bai, M. Elkashlan and A. Nallanathan are with the School of Electronic Engineering and Computer Science, Queen Mary University of London, London E1 4NS, U.K. H. Zhang is with the School of Information and Communication Engineering, Beijing University of Posts and Telecommunications, Beijing, China. J. Wang is with the Department of Electronic Engineering, Tsinghua University, Beijing, 100084, China. C. Xu and L. Hanzo are with the School of Electronics and Computer science, University of Southampton, SO17 1BJ, U.K.

The constructive comments of Prof. Lutz Lampe throughout the preparation of this manuscript are gratefully acknowledged.

L. Hanzo would like to acknowledge the financial support of the Engineering and Physical Sciences Research Council projects EP/N004558/1, EP/P034284/1, EP/P034284/1, EP/P003990/1 (COALESCE), of the Royal Society's Global Challenges Research Fund Grant as well as of the European Research Council's Advanced Fellow Grant QuantCom. J. Wang would like to acknowledge the financial support of National Natural Science Foundation of China (Grant No. 62071268), and of China Postdoctoral Science Foundation (Grant No. 2020T130357). were deregulated [8]. Since then, PLC has evolved rapidly and has been harnessed in various scenarios [1]. Specifically, twoway automatic communication systems [9] were developed in 1982 both for advanced metering and distribution automation. In 1997, the first use of PLC for residential customers' Internet access services was announced by Nortel and Norweb Communications in the U.K, which was capable of providing a data rate of 1 Mbps [10]. Since the early 2000s, PLC has also found applications in domestic broadband applications. For example, products allowing data rates of $14 \mathrm{Mbps}$ (HomePlug 1.0), then $85 \mathrm{Mbps}$ (HomePlug Turbo), and then $200 \mathrm{Mbps}$ (HomePlug AV, HD-PLC, UPA) have become progressively available on the market over the past two decades.

1) Applications of PLC: The applications of PLC are summarized as follows.

- The Smart Grid is a modernized power grid that supports energy transmission and distribution by relying on both wireless and wireline communications techniques [8], [11]. Particularly, PLC plays a unique role in this context. One of its advantages is that the functions of both sensing and communicating can be supported by PLC, because apart from its intrinsic communications function, both power quality and grid device health (e.g., cracked insulators, broken strands, etc.) can be readily revealed by analyzing the communications signals received [12][14]. Another advantage is that the application of PLC in smart grids substantially reduces the cost of both the construction and of the maintenance of the indispensable telecommunications functions in the grid [8]. An additional advantage is that power lines provide a communication path that is completely controlled by the power transmission and distribution sector.

- Smart Factories can also be supported by PLC, which facilitates the interconnection of controllers and motors [1, Ch. 7] [15], [16]. When compared to other alternatives, power lines often represent the shortest routes between the controllers of electronic devices, because the signal passes through the minimum number of relays or gateways [17]. Beneficially, low-latency transmission links relying on PLC become available for missioncritical industrial automotive applications.

- Intelligent Transportation can also be supported by PLC [1, Ch. 10]. Specifically, smart lighting systems are capable of substantially reducing the energy consumption by adapting the lighting intensity on the basis of time and of the residents' demand. However, the geographical extension of street light posts imposes economic challenges 
on deploying the communications infrastructure required [18]. Furthermore, since the car wiring constitutes the third highest factor of their overall weight, immediately after the engine and the gearbox, the power lines can also be exploited for PLC in automatic control and in multimedia streaming by setting up in-vehicle local area networks in cars [19], ships [20] and airplanes [21].

- The Smart Home concept can also be supported by PLC for example by constructing home area networks that interconnect appliances with smart meters or domestic photovoltaic systems both for supporting energy consumption profiling and automatic control [1, Ch. 7] [22].

- Broadband network services can be accessed through PLC links that set up a local area network [23] and/or act as a backbone ${ }^{1}$ for connecting WiFi or $\mathrm{LiFi}^{2}$ networks [1, Ch. 8] [26]-[30]. Furthermore, PLC has the advantage of supporting network coverage in the blind spots of wireless communications, such as underground scenarios [31].

2) Categories of PLC: The applications mentioned in Section I-A1 are fairly diverse and thus rely on different types of PLC systems. From the perspective of their frequency bands, PLC can be generally categorized into three classes, i.e. ultranarrowband (UNB), narrow-band (NB) and broad-band (BB) PLC [11], [32], [33], which are detailed below.

- UNB PLC systems operate in the frequency band of $0.3-$ $3.0 \mathrm{kHz}$. They are capable of providing rates on the order of bits per second (bps) over long distances of $100 \mathrm{~km}$ and more. They are mainly deployed for grid maintenance and monitoring, for example, meter reading in remote areas [11].

- NB PLC systems use the frequency band of $3-500 \mathrm{kHz}$. They are mainly deployed in building automation, transportation control and smart energy management for transmitting control signals and meter reading. Low data-rate NB PLC systems typically have been based on singlecarrier (SC) frequency-shift keying modulation, and provide data rates of $\mathrm{kbps}$, while more recently developed high data-rate NB PLC systems use multi-carrier (MC) modulation and are designed to offer a wider range of data rates up to hundreds of kbps [11], [33].

- BB PLC systems utilize the frequency band of $1.8-$ $100 \mathrm{MHz}$ and deliver up to Gbps-level rates using $\mathrm{MC}$ modulation. They are mainly applied within home areas, for supporting high-rate applications, such as Internet access, online gaming and high-definition television [26].

The smart grid uses the techniques of UNB PLC, of NB PLC, and of BB PLC, while NB PLC is usually invoked in the smart home, in smart factories and in intelligent transportation. Broadband network access services typically exploit BB PLC.

\footnotetext{
${ }^{1}$ Shlezinger et al. [24] have demonstrated that a two-transmit-port-tworeceive-port PLC system is capable of delivering Gbps-level data rates over a frequency range of upto $100 \mathrm{MHz}$ following the configuration of the ITU-T G.9963 standard. This data rate enables PLC to be a competitive candidate for diverse backbone applications. The recent advance of full-duplex techniques [25] also pushes the data rate of PLC even further.

${ }^{2} \mathrm{Li}-\mathrm{Fi}$ (short for light fidelity) is wireless communication technology which utilizes light to transmit data and position between devices.
}

\section{B. Noise in PLC}

The characterization of power line noise dates back to the early 1970s [34], where Smith documented the power spectral density of power line noise both in an urban office and in a rural farm. Following this, various models have been proposed for characterizing the noise in PLC [32], [35]-[79]. In the following, we will briefly introduce both the categories and characteristics of the noise in power lines.

1) Noise Categories in PLC: Signal transmissions in PLC are contaminated by impairments emitted either by electrical devices connected to the power grid or by coupling of radio signals. The impairments are often referred to as noise in parlance and generally categorized into the following five classes shown in Fig. 1 [40], [47], [60].

- Colored background noise is mainly due to spurious emissions from common household appliances and equipment. Its power spectral density (PSD) decays upon increasing the frequency and it is mostly concentrated at frequencies of below $1 \mathrm{MHz}$.

- Narrow-band interference originates from the ingress of broadcast radio signals and thus often has a high power in a narrow bandwidth and fluctuates throughout the day.

- Periodic impulsive noise synchronous with the mains frequency is caused by power supplies, mostly by the switching action of rectifier diodes. It occurs synchronously with the mains cycle and hence has a repetition rate of 50 or $100 \mathrm{~Hz}$, dependent on whether the devices are only affected by the absolute value of the main voltages or also by their polarity [54]. The duration of its impulse is short, usually some microseconds.

- Periodic impulsive noise, which is asynchronous with the alternating current $(A C)$ voltage of the mains frequency, is mainly caused by switching power supplies. It typically has a repetition rate between $50 \mathrm{kHz}$ and $200 \mathrm{kHz}$ and a discrete-line spectrum spaced by the repetition rate.

- Non-periodic asynchronous impulsive noise appears aperiodically, because it typically originates from unpredictable switching transients. The duration of its impulse typically spans from some microseconds upto a few milliseconds [40].

2) Behavior and Impacts of Noise in PLC: In contrast to the noise process in wireless communications, which is usually modeled by a additive white Gaussian noise (AWGN), the noise in PLC typically exhibits complex behavior and severely degrades the integrity of PLC. Let us elaborate further from the perspectives of both the background noise and of the impulsive noise. Specifically, in contrast to the AWGN whose PSD is flat, the PSD of the background noise in PLC typically decreases upon increasing the frequency [45]. In this case, the noise level has to be individually estimated at each subcarrier for the ease of data detection in the system relying on MC modulation. Furthermore, the behavior of impulsive noise in PLC is predominantly characterized by the following four aspects.

- The impulses are usually of high power, hence the desired signals can be severely contaminated in the PLC relying on low-power transmitters. For example, the non-periodic 
Periodic impulsive noise asynchronous to the AC voltage of mains frequency

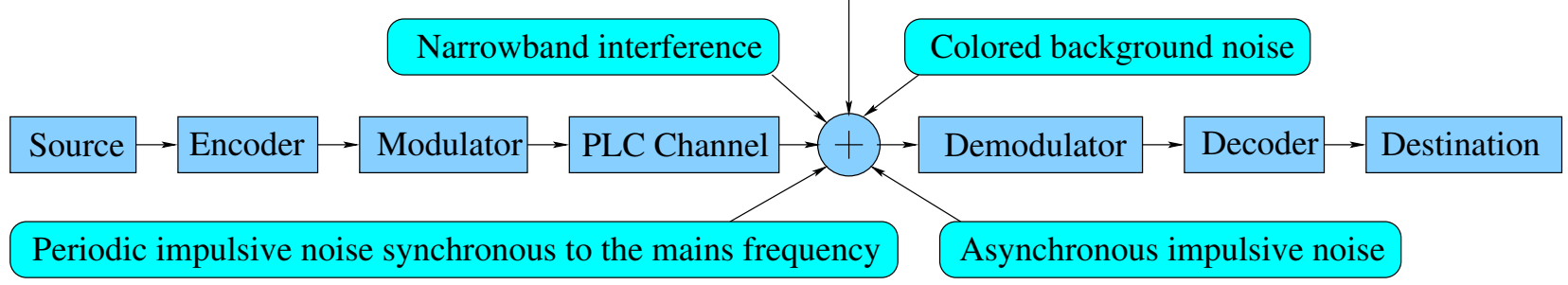

Fig. 1: Illustration of PLC transmission systems contaminated by channels and by diverse noise components.

asynchronous impulsive noise [40] and the periodic impulsive noise appearing at certain frequencies [67] are capable of reaching $50 \mathrm{~dB}$ above the background noise.

- Its samples commonly exhibit non-Gaussian distribution [40], hence the optimality of the detector originally optimized for Gaussian noise environments no longer holds. Therefore, it is imperative to conceive sophisticated detector designs for PLC noise environments.

- It often occurs in form of a burst, which may obliterate multiple packets, because the bursts of errors may exceed the error correction capability of the associated forward error correction (FEC) codes [32]. These error bursts can be beneficially randomized upon dispersing them by interleaving or avoided by automatic repeat-and-request (ARQ). However, some long-duration impulse bursts may last for multiple transmission time slots. This inevitably requires a large number of retransmission attempts or a substantial interleaver depth ${ }^{3}$.

- It is of high occurrence probability, which leads to the fact that PLC transmission links are frequently under the risk of contamination, and hence degrading the reliability of PLC [32].

3) Importance of Noise Characteristics and Modeling: The accurate knowledge of the noise characteristics and the development of noise models have at least three benefits as follows.

- It enables the characterization of the PLC medium and of the associated theoretical limits for data communications.

- It facilitates the design of high-performance transceivers operating reliably over PLC channels, possibly close to the theoretical limits.

- Accurate noise models allow the regeneration of noise samples for simulation testing of PLC systems.

All these benefits motivate us to review the research contributions on the topic of noise modeling and mitigation in PLC.

\section{Prior Work}

A range of excellent surveys has been published on the topic of noise modeling and mitigation in PLC [79]-[82], but they were focused on a specific noise modeling perspective or a

\footnotetext{
${ }^{3}$ The occurrence of error bursts is not unique to the PLC transmission systems. In wireless communications, however, they are usually imposed by fading, while the error bursts in PLC are commonly caused by noise. The behavior difference between the noise process and fading distinguishes PLC from wireless communications.
}

limited number of noise mitigation techniques. To elaborate, Shongwe et al. [80] summarized several simplified mathematical noise models and reviewed the family of nonlinear noise mitigation techniques at the receiver's input along with the benefits of channel coding. Mariyam et al. [81] introduced three impulsive noise models designed for interleavingassisted PLC systems, and reviewed a suite of the nonlinear pre-processing aided noise mitigation techniques used at the receiver's input, along with channel coding and compressedsensing-aided mitigation. Han et al. [79] surveyed the noise models of PLC that are based on measurement campaigns. Laksir et al. [82] provided an inspirational survey of the recent trends in impulsive noise reduction techniques, which categorizes the noise mitigation techniques both at the transmitter and at the receiver based on their error handling mechanisms as well as detection and mitigation strategies. Furthermore, noise modeling and mitigation was also touched upon by the impressive surveys [33], [83]. Specifically, Berger et al. [83] reviewed the salient aspects of multiple-input multiple-output (MIMO)based PLC, including its relationship with the classic wireless MIMO schemes, along with sophisticated signal processing techniques proposed for MIMO PLC. They also touched upon the channel and noise characterization of MIMO PLC solutions. Cano et al. [33] presented an overview of the stateof-the-art in PLC, which covered its potential applications, regulatory and standardization efforts, their channel and noise characterization as well as signal processing techniques. By contrast, our survey provides a comprehensive review of noise modeling from the twin-fold perspectives of empirical as well as of simplified mathematical modeling. More explicitly, we cover ten different noise mitigation techniques by classifying them into noise mitigation at transmitters, as well as both parametric and non-parametric noise mitigation at the receiver. We also cover the pivotal results of joint noise mitigation and channel estimation, portraying the historical evolution of this field in a coherent manner.

\section{Contributions and Organization}

An extensive review of the family of noise modeling and mitigation techniques related to PLC over the past fifty years is presented in this survey. For completeness, we commence with a brief introduction to the channel characteristics and modulation schemes in PLC. Following this, a comprehensive review of representative noise models developed over the past fifty years is presented. Furthermore, an extensive survey of noise mitigation schemes is provided. Additionally, since the 
accuracy of channel estimation in PLC is affected by noise, we review the literature of joint noise mitigation and channel estimation. Finally, a number of research opportunities are outlined for the future work of both noise modeling and mitigation in PLC. Our original contributions are summarized as follows.

- The research advances in noise modeling over the past half-a-century are reviewed. Depending on whether any experimental campaign is involved, we categorize the noise models in PLC into empirical models and simplified mathematical models. Specific to the empirical models, we review the research efforts of all the five noise components of PLC introduced in Section I-B, whilst the family of simplified mathematical models are classified into discontinuous and bursty impulse models, depending on whether the impulse noise events occur in burst.

- We critically review the noise mitigation techniques in PLC, by classifying them into the transmitter and the receiver based techniques. The latter are further categorized into parametric and non-parametric approaches, depending on whether the statistical knowledge of impulsive noise is required. These mitigation techniques are compared in terms of the type of target noise, mitigation performance, spectral efficiency, computational complexity and processing delay.

- Given that the channel estimation is directly impaired by noise in PLC, we appraise a range of techniques conceived for joint noise mitigation and channel estimation, with special emphasis on pilot insertion and iterative estimation.

- A number of future directions and extensions are highlighted for both noise modeling and mitigation in PLC. This may inspire our valued readers in their future work.

Furthermore, noise has also been an crucial issue in some other communications fields, e.g. digital subscriber lines [84], underwater acoustic communications systems [85], and wireless communications [86]. Compared to those in other communications systems, the noise process of PLC - including colored background noise, narrow-band interference and three types of impulsive noise - exhibits the most complex behavior and has attracted substantial research attention. Hence, this article providing a comprehensive survey of noise modeling and mitigation in PLC exemplifies the techniques tackling the noise issues of those systems.

The remainder of this article is organized as follows. In Section II, we briefly introduce the channel characteristics and modulation schemes in PLC. Section III presents our review of noise modeling in PLC, while in Section IV we investigate the family of noise mitigation in PLC. The research advances in joint noise mitigation and channel estimation are presented in Section $\mathrm{V}$, followed by a range of challenges and future research directions in Section VI. Finally, we conclude in Section VII. The glossary is included at the end of this article.

\section{PlC Channels And Modulation Schemes}

For completeness, we continue by briefly introducing the PLC channel characteristics and its modulation schemes.

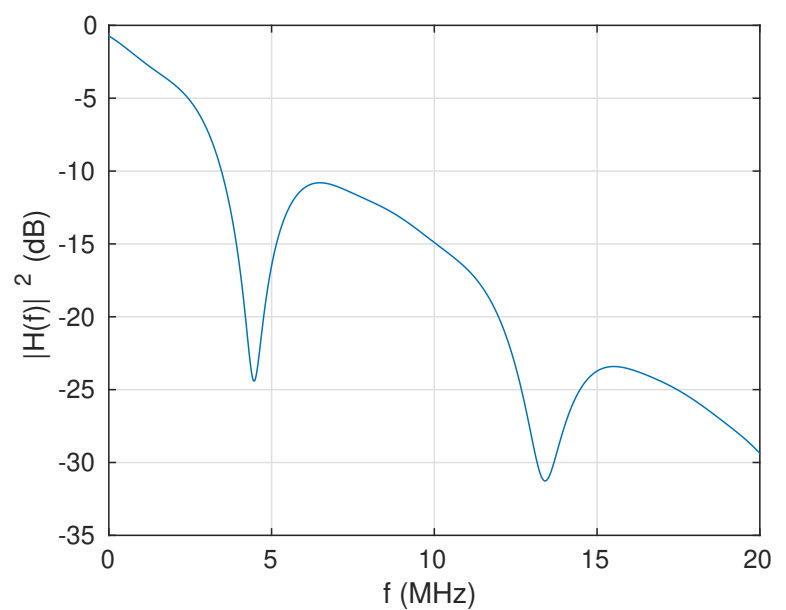

Fig. 2: Illustration of an example of a four-path channel in PLC.

\section{A. Transmission Channel Characteristics}

The early analysis of PLC channel properties dates back to 1980 s [87], where the authors have provided some insightful results on the data transmission characteristics of power lines. Other influential measurement-based contributions include [44], [50], [54], [88]-[95].

Here we briefly summarize the properties of the power-line channel. Firstly, the power-line channel exhibits a frequencydependent low-pass characteristic, imposed by the cable and dielectric losses as well as by radiation effects [94]. Secondly, multi-path propagation is observed in PLC [44], which is caused by signal reflections at cable junctions and by the connection and disconnection of loads. These phenomenological propagation effects can be incorporated into a model that defines a specific frequency response for NB-PLC [32] and for BB-PLC [44], [89]. The reflections also cause further signal attenuation due to an extension of the signal-path lengths [11]. Thirdly, the PLC channel exhibits a linear periodically timevarying (LPTV) nature, which is influenced by the appliances connected. More explicitly, some appliances contain non-linear devices, such as thyristors, whose impedance varies with the mains frequency periodically. In this case, the channel can be modeled by an LPTV process synchronized to the mains [47], [50]. We note that this LPTV model was originally proposed for BB-PLC [50] and it was also confirmed to hold in NB-PLC [93].

An alternative approach is to start from a transmission line theory based presentation of signal propagation, which permits the consideration of specific topologies and load configurations both in BB-PLC [90], [91] and in NB-PLC [96] as well as in MIMO PLC scenarios [97]. Fig. 2 shows a sample frequency response for a power line channel based on the multipath model of [44], for clarifying the channel characteristics of power lines.

\section{B. Modulation in PLC}

As shown in Fig. 1, modulation is a fundamental function block in PLC systems. Here we introduce the types of modulation and demodulation used in PLC, from the perspectives 
of $\mathrm{SC}$ versus $\mathrm{MC}$ as well as coherent versus noncoherent modulation schemes, respectively.

1) Single-Carrier versus Multi-Carrier Modulation: Historically, SC modulation schemes have been adopted in UNB PLC and NB PLC systems, which use bandwidth of a few $\mathrm{kHz}$ [11] and provide low-rate (bps to kbps) services at a low complexity. MC modulation in the form of orthogonal frequency-division multiplexing (OFDM) and wavelet-OFDM may be used for BB PLC [26] and also for NB PLC systems [98]. This allows low-complexity single-tap equalization, when transmitting over the frequency-selective PLC channel. Furthermore, MC modulation facilitates the employment of bit-loading algorithms for each subcarrier, which results in an improved throughput [99]. MC modulation may also be beneficial in the presence of impulsive noise if uncoded modulation is considered, because the impulsive noise power becomes averaged over all subcarriers after the discrete Fourier transform (DFT) block [32].

2) Coherent versus Noncoherent Modulation: Data can be transmitted via phase-shift keying (PSK) and quadrature amplitude modulation (QAM) in both SC and MC modulation schemes that rely on coherent detection, or via frequency-shift keying (FSK) and differential PSK (DPSK) using noncoherent detection. The advantages of the latter are that no explicit channel estimation and no accurate carrier-frequency synchronization are required, thus generally less complex receiver structures and less pilot overhead are required [100]. On the other hand, a signal-to-noise ratio (SNR) penalty is incurred. For these reasons, noncoherent detection is typically applied in low data-rate PLC systems and when the transmission of relatively short data packets is desired [32].

\section{NOISE MODELING}

The accurate knowledge of noise characteristics in PLC is a prerequisite for designing a high-performance transceiver for establishing a reliable transmission link over electric wires. However, the derivation of analytic expressions for empirical noise models in PLC is quite a challenge [49]. Therefore, almost all existing models are established by curve fitting based on measurement results. Early characterization of power line noise dates back to early 1970s [34]. Following this, various empirical models have been proposed for characterizing the noise in PLC [32], [35]-[79]. The advantage of empirical models is to accurately reflect the characteristics of noise, but unfortunately they do not lend themselves to convenient performance analysis and system design, because often intractable functions have to be invoked for pursuing a high modeling accuracy. To overcome this hindrance, various simplified mathematical models have been proposed [65], [80], [101]-[121]. In this section, we briefly introduce the characteristic parameters of noise process in PLC, and then review both the empirical modeling and simplified mathematical modeling options, which are detailed below.

\section{A. Characteristic Parameters of Noise}

As described in Section I-B, the different noise components exhibit different behavior. In this subsection, we present their

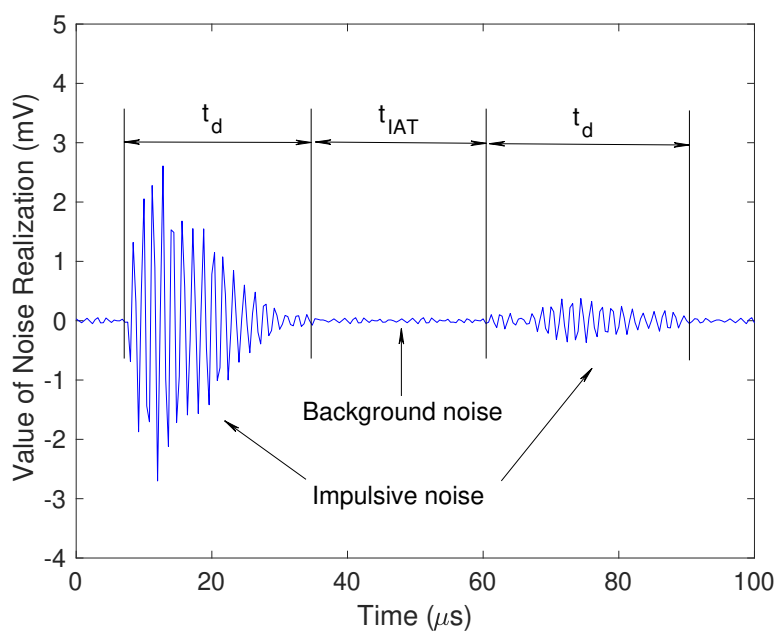

Fig. 3: Illustration of an example of analog noise process in PLC [60], where $t_{d}$ and $t_{\mathrm{IAT}}$ denote the duration and inter-arrival time of impulses, respectively.

characteristic parameters. Specifically, since the background noise in PLC is a superposition of diverse noise components, its realization can be modeled as a collection of $J_{B}$ sinusoids, given by:

$$
n_{B}(t)=\sum_{j=1}^{J_{B}} A_{B}^{j} \sin \left(2 \pi f_{B}^{j} t+\alpha_{B}^{j}\right),
$$

where the random variables of $A_{B}^{j}, f_{B}^{j}$ and $\alpha_{B}^{j}$ are the amplitude, "pseudo-frequency" and phase of the $j$-th sinusoid, respectively. Having a high $f_{B}^{j}$ means that the background noise has a higher power spectral density at the high frequencies. Furthermore, given that the background noise exhibits a non-flat frequency-domain representation [40], the power spectral density denoted by $S_{B}(f)$ is used for characterizing its spectral behavior. In typical PLC systems, $S_{B}(f)$ decreases upon increasing the operational frequency.

The impulsive noise process is a collection of $J_{I}$ decaying sinusoids [53], where the sinusoid functions are multiplied by exponential functions. Furthermore, since impulsive noise occurs discontinuously, the corresponding rectangular functions affect the impulsive noise process as follows:

$$
\begin{array}{r}
n_{I}(t)=\sum_{i=1}^{\infty} \sum_{j=1}^{J_{I}} A_{I}^{j} \sin \left(2 \pi f_{I}^{j}\left(t-t_{\mathrm{arr}}^{i}\right)+\alpha_{I}^{j}\right) \\
\quad \times \exp \left(\frac{t-t_{\mathrm{arr}}^{i}}{\tau_{I}^{j}}\right) \times \sqcap\left(\frac{t-t_{\mathrm{arr}}^{i}}{t_{d}^{i}}\right),
\end{array}
$$

where again the random variables $A_{I}^{j}, f_{I}^{j}$ and $\alpha_{I}^{j}$ represent the amplitude, "pseudo-frequency" and phase of the $j$-th decaying sinusoid, respectively. A higher $A_{I}^{j}$ implies that the impulsive noise may overwhelm the signal received. Furthermore, $\tau_{I}^{j}$ corresponds to the damping factor; the rectangular function $\sqcap\left(t / t_{d}^{i}\right)$ represents the square pulse duration of $t_{d}^{i}$ having a constant amplitude in the interval of $0<t \leqslant 1$ and the amplitude of 0 elsewhere; finally, $t_{\text {arr }}^{i}$ denotes the arrival time of the $i$-th impulsive noise burst. As shown in Fig. 3, $t_{\mathrm{IAT}}=t_{\mathrm{arr}}^{i}-t_{\mathrm{arr}}^{i-1}-t_{d}^{i-1}$ denotes the inter-arrival time between 
two consecutive impulsive noise bursts. Note that $t_{\text {IAT }}$ is of a constant value for the periodic impulsive noise, while it becomes a random variable for the non-periodic asynchronous impulsive noise.

Based on the above-mentioned variables, a range of additional characteristic parameters can be obtained for providing further insights into the associated performance analysis and system design. One is the background-to-impulsive noise power ratio. Upon denoting the PSD of impulsive noise by $S_{I}(f)$, we may formulate the background-to-impulsive noise power ratio as:

$$
\kappa(f)=\frac{S_{B}(f)}{S_{I}(f)},
$$

which is used for characterizing the power ratio between the background noise and the impulsive noise at the frequency $f$. Having a lower value of $\kappa(f)$ implies that the impulsive noise has a higher PSD at the frequency $f$. The other one is the ratio of the average burst-duration to the average inter-burst duration, which is given by [117]:

$$
\Lambda=\frac{\mathbb{E}\left[t_{d}\right]}{\mathbb{E}\left[t_{\mathrm{IAT}}\right]},
$$

where $\mathbb{E}[x]$ represents the expectation of $x$. This can be used for characterizing the ratio between the average temporal interval duration impaired by impulsive noise and that is free from the impulsive noise.

\section{B. Empirical Modeling}

As mentioned in Section I-B1, the noise in PLC can be generally classified into five categories, which are discussed by considering their models as follows.

1) Colored background noise: Several classic models of the PLC's background noise are introduced below, in terms of the sample-value of their noise realization and spectrum. Bearing in mind that the signal can be transmitted using either one-dimensional or two-dimensional modulation schemes in PLC [3], we discuss modeling of the sample-value of their noise realization in the context of these two cases individually. Specifically, the sample-value of one-dimensional noise realization is commonly modeled by a zero-mean Gaussian distribution [42], [45], [74], whose probability density function (PDF) is given by:

$$
f_{A_{B}}(x, f)=\frac{1}{\sqrt{2 \pi \sigma_{n, B}^{2}(f)}} \exp \left(-\frac{x^{2}}{2 \sigma_{n, B}^{2}(f)}\right),
$$

where $\sigma_{n, B}^{2}(f)=S_{B}(f)$ represents the variance of the noise at the frequency of $f$. As a further extension of the one-dimensional noise realization, the magnitude of the twodimensional noise is typically modeled by a Rayleigh PDF [38]:

$$
f_{\left|A_{B}\right|^{2}}(x, f)=\frac{x}{\sigma_{n, B}^{2}(f)} \exp \left(-\frac{x^{2}}{2 \sigma_{n, B}^{2}(f)}\right),
$$

where $\sigma_{n, B}^{2}(f)$ is the variance of the real and imaginary noise voltage value. Another model of the two-dimensional noise's magnitude is the Nakagami- $m$ distribution [49], [56], whose PDF is formulated as:

$$
f_{\left|A_{B}\right|^{2}}(x, f)=\frac{2}{\Gamma(m)}\left(\frac{m}{\Omega(f)}\right)^{m} x^{2 m-1} \exp \left(-\frac{m x^{2}}{\Omega(f)}\right),
$$

where $\Gamma(\cdot)$ is the Gamma function [122]; $\Omega(f)$ represents the mean power of $x$ at the frequency $f ; m=\left(\mathbb{E}\left[x^{2}\right]\right)^{2} / \operatorname{var}\left[x^{2}\right]$. Note that the Nakagami- $m$ distribution is exactly the same as the Rayleigh distribution, if we set $m=1$.

The measurement results of the background noise's spectrum are often fitted into a negative exponentially decaying function [122]. Specifically, Hooijen [39] modeled the PSD in an exponential form, given by:

$$
S_{B}(f)=10^{\left(K-3.95 \times 10^{-5} f\right)}[\mathrm{W} / \mathrm{Hz}],
$$

where $f$ is the frequency in Hz. $K$ slowly changes over time and resembles the Gaussian distribution associated with the mean value of 5.64 and with the variance value of 0.25 . Furthermore, Phillips [41] formulated the model as a first order exponential function, whose amplitude spectral density is expressed as:

$$
S_{B}(f)=S_{0}+S_{1} \exp \left(-\frac{f}{f_{1}}\right)
$$

where $\left(S_{0}, S_{1}, f_{1}\right)=(-35,35,3.6)$ for residential environments and $\left(S_{0}, S_{1}, f_{1}\right)=(-33,40,8.6)$ for industrial environments; $f$ is the frequency in $\mathrm{MHz}$; the amplitude of $S_{B}(f)$ is in $\mu \mathrm{V}(\mathrm{dB}) / \mathrm{Hz}^{1 / 2}$. Note that $\mathrm{V}^{2} / \mathrm{Hz}$ is usually used as the unity of the PSD and hence $\mathrm{V} / \mathrm{Hz}^{1 / 2}$ here is used for representing the corresponding amplitude spectral density. Furthermore, Esmailian et al. [45] proposed a three-parameter model, which is given by:

$$
S_{B}(f)=a+b|f|^{c}[\mathrm{dBm} / \mathrm{Hz}],
$$

where $f$ is the frequency in $\mathrm{MHz}$. Here we have $(a, b, c)=$ $(-140,38.75,0.720)$ for the best case and $(a, b, c)=$ $(-145,53.23,-0.337)$ for the worse case. Additionally, the attempts of applying MIMO techniques in PLC [123] inspires the modeling of the MIMO PLC's background noise. It can be characterized in both the frequency domain [62], [66] and in the time domain [69]. In Table I, we summarize the influential modeling efforts concerning the empirical background noise, from the perspectives of its voltage, environment, frequency band, the sample-value of its noise realization and its spectral behavior.

2) Narrow-band interference: Narrow-band interference is a typical impairment imposed by uncoordinated PLC devices and uncoordinated man-made appliances [32]. It can be characterized in terms of the sample-value of its realization, as well as both by its temporal behavior and spectral behavior. Specifically, Philipps [41] confirmed that its sample-value of realization obeys the lognormal distribution in both residential and industrial environments. The temporal behavior of narrowband interference in PLC was investigated in [121] based on the data measured both in Italy and China, which revealed that 
TABLE I: Milestone papers on background noise modeling. Here CDF corresponds to cumulative density function; MIMO refers to multiple-input multipleoutput PLC channels; AR represents auto-regressive model. The "Dash" here means that the article does not deal with the subject in the column.

\begin{tabular}{|c|c|c|c|c|c|c|}
\hline Year & Paper & Voltage & Environment & Frequency & Sample value of noise realization & Spectrum \\
\hline 1972 & Smith [34] & low & office/industry & $10 \mathrm{kHz}-1 \mathrm{GHz}$ & Rayleigh & empirical \\
\hline 1997 & Arzberger et al. [38] & low & - & $20-150 \mathrm{kHz}$ & Sum of Rayleigh & empirical \\
\hline 1998 & Hooijen [39] & low & home & $9-95 \mathrm{kHz}$ & - & (8) \\
\hline 2000 & Philips [41] & low & office and home & $0-30 \mathrm{MHz}$ & - & (9) \\
\hline 2002 & Cooper and Jeans [42] & low & home & $9-148.5 \mathrm{kHz}$ & Gaussian & (8) \\
\hline 2003 & Esmailian et al. [45] & low & office and home & $1-30 \mathrm{MHz}$ & Gaussian & (10) \\
\hline 2003 & Benyoucef [46] & low & office and home & $1-30 \mathrm{MHz}$ & - & (9) \\
\hline 2003 & Tang et al. [48] & low & home & $1-10 \mathrm{MHz}$ & empirical CDF & (8) \\
\hline 2005 & Meng et al. [49] & low & lab and home & $1-30 \mathrm{MHz}$ & Nakagami-m & empirical \\
\hline 2007 & Tao et al. [56] & medium & substation & $40 \mathrm{kHz}-2 \mathrm{MHz}$ & Nakagami-m & (9) \\
\hline 2007 & Tsuzuki et al. [57] & low & cargo ships & $0-100 \mathrm{MHz}$ & - & empirical \\
\hline 2010 & Cortes et al. [60] & low & lab and office & $0-25 \mathrm{MHz}$ & Gaussian & empirical \\
\hline 2010 & Andreadou et al. [61] & - & - & $0-20 \mathrm{MHz}$ & - & (9) \\
\hline 2011 & Bert et al. [64] & - & - & $0-100 \mathrm{MHz}$ & - & (10) \\
\hline 2012 & Hashmat et al. [66] & low & home (MIMO) & $2-150 \mathrm{MHz}$ & - & (10) \\
\hline 2012 & Hashmat et al. [69] & low & home (MIMO) & $2-150 \mathrm{MHz}$ & - & Vector AR \\
\hline 2013 & Antoniali et al. [71] & low & vehicle & $0-50 \mathrm{MHz}$ & - & white \\
\hline 2016 & Guerrieri et al. [74] & low & vehicle & $2-100 \mathrm{MHz}$ & Gaussian & white \\
\hline
\end{tabular}

narrow-band interference exists all the time in both countries in the low frequency bands below $100 \mathrm{kHz}$. As for the spectral behavior, Benyoucef [46] characterized its spectrum as a superposition of $J_{\mathrm{NI}}$ Gaussian-like functions, formulated as:

$$
S_{\mathrm{NI}}(f)=\sum_{j=1}^{J_{\mathrm{NI}}} A_{j} \exp \left(-\frac{\left(f-f_{0, j}\right)^{2}}{2 B_{j}^{2}}\right),
$$

where $J_{\mathrm{NI}}$ presents the total number of the impairment sources; $A_{j}, f_{0, j}$ and $B_{j}$ denote the amplitude of the interference, the central frequency and the bandwidth of the $j$-th impairment, respectively. These four parameters vary over the time and their statistics are detailed in Table II of [46] for office sites and residential buildings, respectively. Apart from the models based on measurement campaigns, Milioudis et al. [72] theoretically derived the modeling equations with the aid of the field-to-transmission-line coupling equations, given a certain network topology in medium-voltage scenarios. Since the number of research contributions dealing with narrow-band interference is limited, they are not compared in tables.

3) Periodic impulsive noise synchronous with the mains frequency: Given that its fixed inter-arrival time is observed [124], the periodic noise synchronous with mains is typically characterized by using its sample-value of noise realization, spectrum and noise duration. Specifically, Vines et al. [7] investigated the properties of periodic noise originated from universal motors and light dimmers as well as other noise sources on residential power distribution circuits. Katayama et al. [55] proposed a noise model based on the temporal cyclostationary Gaussian process, where the mean value of the noise was zero and its variance varied synchronously with the mains. The PDF of the sample-value of its noise realization is given by

$$
f_{A_{\mathrm{P} 2}}(x(t, f))=\frac{1}{\sqrt{2 \pi \sigma_{\mathrm{P} 2}^{2}(t, f)}} \exp \left(-\frac{(x(t, f))^{2}}{2 \sigma_{\mathrm{P} 2}^{2}(t, f)}\right),
$$

where we have $\sigma_{\mathrm{P} 2}^{2}(t, f)=\sigma_{\mathrm{P} 2}^{2}(t) S_{\mathrm{P} 2}(f)$. Here, upon denoting its period, impulse duration and arrival time by $T_{\mathrm{P} 2}, \tau_{\mathrm{P} 2}$ and
$t_{\mathrm{P} 2}$, respectively, we have

$$
\sigma_{\mathrm{P} 2}^{2}(t)=\left\{\begin{array}{l}
A_{\mathrm{P} 2}, t \in\left[t_{\mathrm{P} 2}+m T_{\mathrm{P} 2}, t_{\mathrm{P} 2}+\tau_{\mathrm{P} 2}+m T_{\mathrm{P} 2}\right] \\
0, \quad \text { otherwise }
\end{array}\right.
$$

where $m$ represents the $m$-th occurrence of the periodic impulsive noise; upon denoting the duration of an AC cycle by $T_{\mathrm{AC}}$, its period is given by $T_{\mathrm{P} 2}=0.5 T_{\mathrm{AC}}$ [55]. The spectrum was represented using a linearly time-invariant (LTI) system, which was expressed as an exponential function [55]:

$$
S_{\mathrm{P} 2}(f)=\frac{a}{2} \exp (-a|f|) .
$$

Note that the difference between (5) and (12) is that the variance of (12) fluctuates over time due to its cyclostationary behavior. Similarly, Canete et al. [50] modeled the periodic impulsive noise as a superposition of noise components originated from various sources, where each noise component had a specific spectrum. Note that the spectra in [50], [55] were modeled using fixed spectral shapes. In practice, however, the spectra of periodic impulse noise processes exhibits timevarying behavior [32].

To pursue a higher modeling accuracy, Nassar et al. proposed a multiple-filter aided model [67]. In this model, a given period of time is divided into $J_{P 2}$ intervals $\mathcal{R}_{1}, \mathcal{R}_{2}, \ldots, \mathcal{R}_{J_{P 2}}$. Assuming that the noise is stationary in each interval $\mathcal{R}_{j}$, the noise is represented as the response of a LPTV system denoted by $h[k, \tau]$ to a stationary input of $s[k]$, given by:

$$
n_{\mathrm{P} 2}[k]=\sum_{\tau} h[k, \tau] s[\tau]=\sum_{j=1}^{J_{P 2}} \mathbf{1}_{k \in \mathcal{R}_{j}} \sum_{\tau} h_{j}[\tau] s[\tau],
$$

where $\mathbf{1}_{\mathcal{A}}$ is the indicator function $\left(\mathbf{1}_{\mathcal{A}}=1\right.$ if $\mathcal{A}$ is true, 0 otherwise) and $J_{P 2}$ represents the number of noise spectral shapes; we have $h[k, \tau]=\sum_{j=1}^{J_{P 2}} h_{j}[\tau] \mathbf{1}_{k \in \mathcal{R}_{j}}$. Similarly, Gianaroli et al. [70] developed the periodic noise generator based on the deseasonalized auto-regressive moving average (DARMA) model [125], [126]. Specifically, the autoregressive moving average (ARMA) model [127, Ch. 3] is usually used for describing a stationary stochastic process. 
TABLE II: Milestone papers on modeling periodic impulsive noise, where LTI and LPTV represent linearly time-invariant and linearly periodic time-varying, respectively, and DARMA refers to deseasonalized auto-regressive moving average process. The "Dash" here means that the article does not deal with the subject in the column.

\begin{tabular}{|c|c|c|c|c|c|c|c|}
\hline Year & Paper & Voltage & Environment & Frequency & Sample value of noise realization & Spectrum & Duration \\
\hline 1984 & Vines et al. [36] & low & home & $5-100 \mathrm{kHz}$ & - & empirical & - \\
\hline 2002 & Cooper et al. [42] & low & home & $9-148.5 \mathrm{kHz}$ & Gaussian & LTI & - \\
\hline 2005 & Canete et al. [50] & low & indoor & $0-20 \mathrm{MHz}$ & Gaussian Mixture & LTI & - \\
\hline 2005 & Suljanovic et al. [51] & high & corona & $150-3500 \mathrm{kHz}$ & Gaussian & LPTV & - \\
\hline 2006 & Corripio et al. [54] & low & indoor & $1-20 \mathrm{MHz}$ & - & LTI & - \\
\hline 2006 & Katayama et al. [55] & - & - & $3-450 \mathrm{kHz}$ & Gaussian & LTI & - \\
\hline 2010 & Cortes et al. [60] & low & indoor & $1-30 \mathrm{MHz}$ & - & empirical & - \\
\hline 2012 & Nassar et al. [67] & low/medium & smart grid & $3-500 \mathrm{kHz}$ & Gaussian & LPTV & empirical \\
\hline 2012 & Barmada et al. [68] & low & electric vehicle & $0-30 \mathrm{MHz}$ & - & - & - \\
\hline 2012 & Gianaroli et al. [70] & low & indoor & $1-30 \mathrm{MHz}$ & Gaussian & DARMA & - \\
\hline \multirow{2}{*}{2013} & \multirow{2}{*}{ Antoniali et al. [71] } & \multirow{2}{*}{ low } & \multirow{2}{*}{ electric vehicle } & $3-500 \mathrm{kHz}$ & - & analytical & - \\
\hline & & & & $2-100 \mathrm{MHz}$ & - & empirical & - \\
\hline 2016 & Guerrieri et al. [74] & low & vehicle & $10 \mathrm{kHz}-40 \mathrm{MHz}$ & - & - & empirical \\
\hline
\end{tabular}

However, the cyclostationary nature of the periodic impulsive noise prohibits the direct employment of ARMA models. In order to overcome this issue, the "deseasonalization" technique of [127, Ch. 13] is invoked for removing associated cyclostationary component in the periodic noise and for producing a stationary sequence. Then we may characterize the noise using the ARMA model, which can be represented by:

$$
n_{\mathrm{P} 2}[k]=s[n]-\sum_{j=1}^{q} \theta[j] x[k-j]+\sum_{i=1}^{p} \phi[i] n_{\mathrm{P} 2}[k-i],
$$

where $s[k]$ is a real-value white Gaussian process, whose samples have zero mean and a certain variance. Furthermore, $\{\theta[j]\}_{j=1}^{q}$ and $\{\phi[i]\}_{i=1}^{p}$ are the parameters of the $q$-th order MA and the $p$-th order AR parts, respectively. These two sets of parameters can be computed recursively by invoking the innovations algorithm of [128] and Yule-Walker equations of [127], respectively. Then the seasonalization technique [127, Ch. 12], which is the inverse process of the deseasonalization technique, is applied to restore the cycrostationary sequence.

Apart from the afore-mentioned modeling efforts, periodic impulsive noise synchronous to the mains is also investigated numerically in the literature [60], [68], [71], [74], [75]. Specifically, Cortes et al. [60] revealed that the periodic noise having the repetition rate of $100 \mathrm{~Hz}$ was more harmful in BB-PLC than the one having the repetition rate of $50 \mathrm{~Hz}$, because the latter one was usually restricted within the frequency range of $0-1 \mathrm{MHz}$, while the former one appeared at the higher frequencies. Moreover, it was shown that the periodic noise in electric vehicles was mainly caused by the motor drive and by AC/DC converter in [68], [71], [74]. Furthermore, the distribution of the high voltage periodic noise was evaluated in [75]. In Table II, we summarized the influential research contributions on the modeling of periodic impulsive noise synchronous with the mains.

4) Periodic impulsive noise asynchronous with the mains frequency: Given its periodic nature, the periodic impulsive noise processes that are asynchronous with the mains frequency can also be characterized using their amplitude, duration, period and spectrum. It was revealed in [59] that the periodic impulsive noise asynchronous with the mains typically has much lower amplitude and duration, but much higher repetition rate than those of the synchronous noise components. However, apart from their different cyclic periods, the periodic impulsive noise processes that are asynchronous or synchronous with the mains frequency have no substantial difference in terms of their temporal behavior [79]. Therefore, the periodic impulsive noise processes asynchronous with the mains frequency can also be modeled by the cyclostationary processes detailed in Section III-B3.

Furthermore, the corona noise caused by high-voltage electric wires and by electromagnetic disturbances also belong to the category of periodic impulsive noise that is asynchronous with the mains. Specifically, its period was characterized using a fixed inter-arrival time and its spectrum was represented using a certain shape of the PSD. As for the sample-value of its noise realization, it was modeled by the Gaussian distribution [51]. Moreover, Guezgouz et al. [63] modeled the electromagnetic disturbances imposed by printers on the PLC network using a sum of two damped sinusoidal functions, given by:

$$
\begin{aligned}
n_{\mathrm{P} 1}(t)=A_{1}^{\mathrm{P} 1} & \sin \left(\omega_{1}^{\mathrm{P} 1} t\right) \times \exp \left(-\alpha_{1}^{\mathrm{P} 1} t\right) \\
& +A_{2}^{\mathrm{P} 1} \sin \left(\omega_{2}^{\mathrm{P} 1} t\right) \times \exp \left(-\alpha_{2}^{\mathrm{P} 1} t\right),
\end{aligned}
$$

where $A_{1}^{\mathrm{P} 1}=0.058, A_{2}^{\mathrm{P} 1}=0.01, \omega_{1}^{\mathrm{P} 1}=2 \pi \cdot 11 \cdot 10^{6} \mathrm{rad} / \mathrm{s}$, $\omega_{2}^{\mathrm{P} 1}=2 \pi \cdot 26 \cdot 10^{6} \mathrm{rad} / \mathrm{s}, \alpha_{1}^{\mathrm{P} 1}=5 \cdot 10^{6}$ and $\alpha_{2}^{\mathrm{P} 1}=2$. $10^{6}$. Since the number of the research contributions dealing with periodic impulsive noise asynchronous with the mains frequency is limited, they are not compared in tables.

5) Non-periodic asynchronous impulsive noise: In contrast to the periodic impulsive noise, the non-periodic asynchronous impulse noise exhibits random values of duration and IAT. Hence it has to be characterized using more variables, including the sample-value of noise realization, duration and IAT. The early investigation on mechanism of non-periodic asynchronous impulsive noise dates back to late 1970s [35]. In the following, we present some commonly-used models individually.

The sample-value of its noise realization was modeled by the Gaussian distribution in [43], [52], [53], [58], [65] and its PDF is given by (5). Furthermore, the sample-value of its 
TABLE III: Milestone papers on modeling non-periodic asynchronous impulsive noise, where IAT represents inter-arrival time and AR refers to auto-regressive process. The "Dash" here means that the article does not deal with the subject in the column.

\begin{tabular}{|c|c|c|c|c|c|c|c|c|c|}
\hline Year & Paper & Voltage & Environment & Frequency & Sample Value & Duration & IAT & Temporal Corr. & Spectrum \\
\hline 1979 & [35] & low & residential & $10 \mathrm{kHz}-1 \mathrm{GHz}$ & empirical & - & - & - & - \\
\hline 1989 & [37] & low & intra-building & $30-80 \mathrm{kHz}$ & Rayleigh & empirical & empirical & - & - \\
\hline 2002 & [40] & medium/low & substation/apartment & $0.2-20 \mathrm{MHz}$ & empirical & Exponential & Exponential & Partitioned Markov & $\mathrm{AR}$ \\
\hline 2002 & [43] & low & indoor & $1-20 \mathrm{MHz}$ & Gaussian & Weibull & Weibull & - & - \\
\hline 2003 & [45] & low & office and home & $1-30 \mathrm{MHz}$ & Beta-like & Gaussian Mixture & Gamma & - & - \\
\hline 2006 & [52] & low & vehicle & $4-21 \mathrm{MHz}$ & Gaussian & empirical & empirical & empirical & - \\
\hline 2006 & [53] & low & indoor & $1-20 \mathrm{MHz}$ & Gaussian & constant & exponential & - & - \\
\hline 2008 & [58] & low & vehicle & $0-40 \mathrm{MHz}$ & Gaussian & empirical & empirical & - & empirical \\
\hline 2011 & [64] & low & home & $0-100 \mathrm{MHz}$ & Middleton & Exponential & Exponential & - & - \\
\hline 2011 & [65] & low & home & $0-100 \mathrm{MHz}$ & Gaussian & Exponential & Exponential & - & - \\
\hline 2014 & [73] & high & Substation & $800 \mathrm{MHz}-2.5 \mathrm{GHz}$ & Gaussian & Exponential & Exponential & Partitioned Markov & empirical \\
\hline 2016 & [75] & low & home appliances & $0-100 \mathrm{MHz}$ & empirical & empirical & - & - & empirical \\
\hline
\end{tabular}

noise realization may also be modeled to resemble the Betalike distribution, whose PDF is formulated to be [45]:

$$
f_{A_{\mathrm{A}}}(x)=\frac{\left(\frac{x-8}{9}\right)^{2}\left(\frac{17-x}{9}\right) \Gamma(5)}{\Gamma(3)}, 8 \leq x \leq 17 \mathrm{mV}
$$

In terms of the duration of $t_{d}$ and IAT of $t_{\mathrm{IAT}}$, they are commonly modeled to obey the exponential distribution [40], [53], [64], [65], whose PDF is given by [65]:

$$
f_{t_{d(\mathrm{IAT})}}(t)=\lambda_{d(\mathrm{IAT})} \exp \left(-\lambda_{d(\mathrm{IAT})} t\right),
$$

where $\lambda_{d(\mathrm{IAT})}$ is the impulsive noise's duration or IAT. Additionally, the Gaussian mixture distribution and the Gamma distribution were proposed for $t_{d}$ and $t_{\text {IAT }}$ [45], respectively, while Schiffer [129] modeled $t_{\text {IAT }}$ using a Poisson process.

Given that impulse-free and impulse-infested temporal intervals appear sequentially in PLC systems, we may also use a two-state Markov chain to model this noise process. However, it was revealed in [40] that the statistics of the impulse duration and of the IAT may be modeled by the superposition of several exponential distributions associated with different mean values. Based on this observation, Zimmermann and Dostert [40] proposed a noise model with the aid of the so-called partitioned Markov chains, which are capable of carefully balancing the modeling accuracy and its adaptability. Specifically, the temporal behavior of the noise process is represented using $J$ noise states $Z_{j}, \forall j=\{1, \cdots, J\}$, which are partitioned into two groups: the impulse-free states $Z_{j}$, $\forall j=\{1,2, \cdots, v\}$ corresponding to the IAT, and the impulseinfested states $Z_{j}, j=\{v+1, v+2, \cdots, J\}$ corresponding to the impulse duration. The values in each state of both groups obey the exponential distribution associated with a specific mean value. The transition probability among the states can be described using the matrix $\boldsymbol{U}$ for impulse-free states and by the matrix $\boldsymbol{G}$ for the impulse-impaired states, which are given below:

$$
\boldsymbol{U}=\left[\begin{array}{ccccc}
u_{1,1} & 0 & \cdots & 0 & u_{1, G} \\
0 & u_{2,2} & \ddots & \vdots & u_{2, G} \\
\vdots & \ddots & \ddots & 0 & \vdots \\
0 & \cdots & 0 & u_{v, v} & u_{v, G} \\
u_{G, 1} & u_{G, 2} & \cdots & u_{G, v} & 0
\end{array}\right]
$$

and

$$
\boldsymbol{G}=\left[\begin{array}{ccccc}
g_{1,1} & 0 & \cdots & 0 & g_{1, U} \\
0 & g_{2,2} & \ddots & \vdots & g_{2, U} \\
\vdots & \ddots & \ddots & 0 & \vdots \\
0 & \cdots & 0 & g_{w, w} & g_{w, U} \\
g_{U, 1} & g_{U, 2} & \cdots & g_{U, w} & 0
\end{array}\right]
$$

where the element $u_{j, j^{\prime}}$ represents the transition probability from the state $Z_{j}$ to the state $Z_{j^{\prime}}$; more particularly $u_{j, G}$ refers to the transition probability from the specific impulsefree state $Z_{j}$ to any of the impulse-impaired states, while $u_{G, j}$ corresponds to the transition probability from any of the impulse-impaired states to the particular impulse-free state $Z_{j}$.

Apart from these models represented by analytical expressions, the asynchronous impulsive noise has also been investigated based on straightforward observations. For example, Degardin et al. [58] evaluated the nature of impulsive noise of in-vehicle power lines in both static and dynamic conditions, which revealed that the pulse amplitude and the pulse duration of in-vehicle PLC were smaller than those of in-house PLC. Moreover, considering that plug-in/out and on/off-switching events of home appliances were the main source of the asynchronous impulsive noise in indoor PLC, Antoniali et al. [75] assessed the behavior of impulses originated from various home appliances, which indicated that the aperiodic impulsive noise was caused by plug-in events and by a small fraction of unpluging events. Furthermore, the noise from appliances was also investigated for indoor PLC [49], which implied that most appliances contributed no noise to the broad-band frequencies, except light-dimmers and appliances equipped with motors. In Table III, we summarize the seminal research contributions on modeling non-periodic asynchronous impulsive noise.

Remark 1. The behavior of the noise in NB-PLC significantly deviates from that in BB-PLC. Specifically, both colored background noise and narrow-band interference as well as periodic impulsive noise synchronous with the mains frequency are dominant in NB-PLC [32], [55]. By contrast, both periodic impulsive noise and non-periodic asynchronous impulsive noise as well as narrow-band interference impose substantial impact on BB-PLC systems, because their power tends to remain much higher than that of the background noise as observed in [60]. 


\section{Simplified Mathematical Modeling}

The empirical modeling detailed in Section III-B allows us to characterize the noise in a straightforward manner. However, due to the usage of uncommon functions, some empirical models may not lend themselves to convenient mathematical analysis. To tackle this problem, simplified mathematical noise models have also been proposed [65], [80], [101]-[121]. Fig. 4 provides a classification of the popular noise modeling techniques. From the perspective of impulse duration, simplified mathematical modeling may be applied to discontinuous impulses and bursty impulses, which are detailed below.

1) Discontinuous Impulses: Discontinuous impulse modeling is the simpler one of the above-mentioned two modeling approaches. In general, discontinuous impulse modeling is applicable for modeling the impulsive noise after interleaving. As shown in Fig. 5a, it ignores the temporal correlation between the impulse-free states and the impulse-infested states, and treats the impulse noise as an independent and identically distributed (i.i.d.) variable. As classified in Fig. 4, discontinuous impulsive noise modeling typically includes the Gaussian Mixture [65], [115], [119], the Bernoulli Gaussian [104], [106], [108], Middleton's Classes [105], [116], [118] and the $\alpha$-stable [113], [114] scenarios, which are introduced individually below.

The Gaussian Mixture Model [65], [115], [119] characterizes the noise process as a mixture of several Gaussiandistributed components. Let us denote the noise value at the $k$-th time instant by $n_{k}$. The PDF of the real-valued noise in the Gaussian Mixture Model is formulated by [65], [115], [119]:

$$
f_{\mathrm{GMM}}\left(n_{k}\right)=\sum_{m=0}^{M-1} \frac{p_{m}}{\sigma_{m} \sqrt{2 \pi}} \exp \left(-\frac{n_{k}^{2}}{2 \sigma_{m}^{2}}\right),
$$

where $p_{m}$ represents the occurrence probability of the $m$-th component and we have $\sum_{m=0}^{M-1} p_{m}=1$; the $m$-th component obeys the Gaussian distribution $\mathcal{N}\left(0, \sigma_{m}^{2}\right)$.

As a special case of the Gaussian Mixture Model, the Bernoulli-Gaussian Model [104], [106], [108] simplifies the noise process to be a two-term Bernoulli process. Mathematically, we have $n_{k}=w_{k}+b_{k} i_{k}$, where $w_{k} \sim \mathcal{N}\left(0, \sigma_{n, B}^{2}\right)$, $i_{k} \sim \mathcal{N}\left(0, \sigma_{n, I}^{2}\right)$ and $b_{k}$ is the Bernoulli random variable. The PDF of the real-valued noise relying on the Bernoulli-Gaussian Model is expressed as [104], [106], [108]:

$$
\begin{aligned}
f_{\mathrm{BGM}}\left(n_{k}\right)= & \frac{1-p}{\sigma_{n, B} \sqrt{2 \pi}} \exp \left(-\frac{n_{k}^{2}}{2 \sigma_{n, B}^{2}}\right) \\
& +\frac{p}{\sigma_{n, I} \sqrt{2 \pi}} \exp \left(-\frac{n_{k}^{2}}{2 \sigma_{n, I}^{2}}\right),
\end{aligned}
$$

where we have $p=\operatorname{Pr}\left(b_{k}=1\right)$, which indicates the occurrence probability of impulsive noise. The Bernoulli-Gaussian Model is widely invoked in the performance analysis and design of communications systems, as an explicit benefit of its simplicity and capability of characterizing the random occurrence of high-power impulses [115].

Further setting $p_{m}=e^{-A} A^{m} / m$ ! and $M-1=\infty$, we may transform the Gaussian-Mixture Model into Middleton's

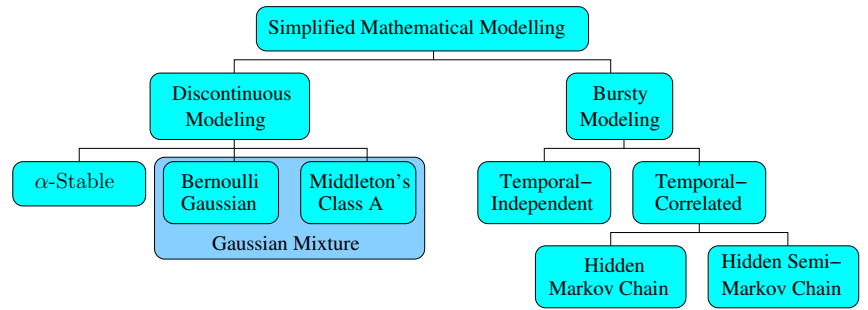

Fig. 4: Illustration of the categories of simplified mathematical noise models.

Class A noise model [103], [105]. The PDF of the real-valued noise relying on Middleton's Class A noise model is given by [105]:

$$
f_{\mathrm{MCA}}\left(n_{k}\right)=e^{-A} \sum_{m=0}^{\infty} \frac{A^{m}}{m ! \sigma_{m} \sqrt{2 \pi}} \exp \left(-\frac{n_{k}^{2}}{2 \sigma_{m}^{2}}\right),
$$

where we have $\sigma_{m}^{2}=\sigma^{2} \cdot(m / A+\kappa) /(1+\kappa) ; \kappa=\sigma_{n, B}^{2} / \sigma_{n, I}^{2}$ is the background to impulsive power ratio [111]; $\sigma^{2}$ represents the overall power of the noise process and is given by $\sigma^{2}=\sigma_{n, B}^{2}+\sigma_{n, I}^{2}=(1+1 / \kappa) \sigma_{n, B}^{2} ; A$ is the product of the impulsive rate and the impulse mean duration. To elaborate a little further in physically tangible terms, Middleton's Class A scenario may be interpreted as the superposition of statistically independent noise components, whose sources obey the Poisson distribution in both space and time [105]. More explicitly, at the $k$-th time instant, the destination has a certain probability of receiving impulses from $m$ sources, where $m$ can be any value between 0 and $\infty$. The PDF of a certain noise sample realization is the average of obtaining this value from $m$ sources. Apart from the Class A noise model, Middleton [105] has also proposed the Class B and Class C models. Among these three classes, Middleton's Class A is the most popular one, due to the fact that Middleton's Class A model is capable of characterizing a wide range of practical impairments [105]. Furthermore, since only the parameters $\left(A, \kappa, \sigma^{2}\right)$ are required for characterizing Middleton's Class A model, this facilitates convenient performance analysis and system design.

The Alpha-Stable distribution constitutes the generalization of the Gaussian distribution and it is also suitable for noise modeling in PLC [113], [114]. The characteristic function of the real-valued noise in the Alpha-Stable model is given by [110], [113], [114]:

$$
f_{\alpha S}\left(n_{k}\right)=\exp \left(i \delta n_{k}-\left|\gamma n_{k}\right|^{\alpha} \Phi\right)
$$

and

$$
\Phi= \begin{cases}1+i \beta \operatorname{sgn}\left(n_{k}\right) \tan \left(\frac{\pi \alpha}{2}\right), & \text { if } \alpha \neq 1, \\ 1-i \beta \operatorname{sgn}\left(n_{k}\right) \frac{2}{\pi} \log \left|n_{k}\right|, & \text { if } \alpha=1,\end{cases}
$$

where $i$ is the imaginary unit and $\operatorname{sgn}(x)$ is a sign function of $x$. We have $-\infty<\delta<+\infty, \gamma>0,0<\alpha \leqslant 2,-1 \leqslant$ $\beta \leqslant 1$. Furthermore, $\gamma$ is a scaling parameter, which controls the spread of the samples, and $\alpha$ determines the degree of impulsiveness. A smaller the value of $\alpha$ implies both a higher occurrence probability of and a larger amplitude of extreme pulses. Particular to the case of $\beta=0$, the distribution is symmetric about the center $\delta$ and is termed by the symmetric $\alpha$-stable $(\mathrm{S} \alpha \mathrm{S})$ [110]. 


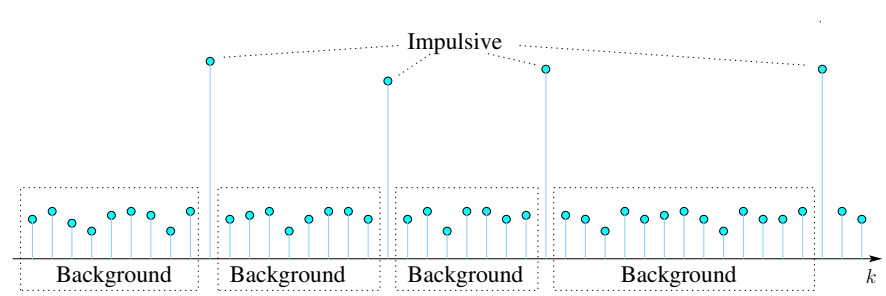

(a)

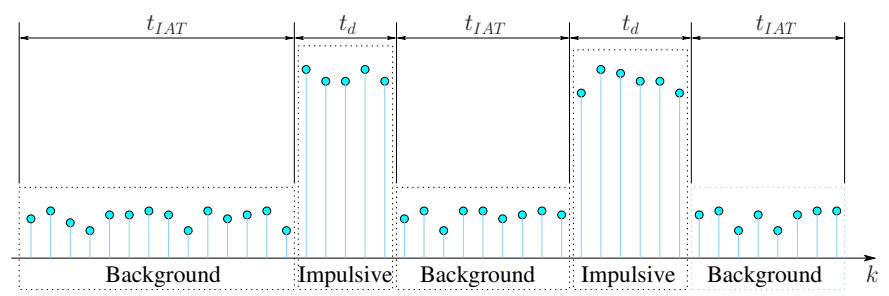

(b)

Fig. 5: Illustration of discontinuous and of bursty impulses. (a) Discontinuous impulses; (b) Bursty impulses.

2) Bursty Impulses: As commented in Section III-C1, the discontinuous modeling technique can only be used for modeling the noise process after interleaving. To characterize the noise process in a wider range of scenarios, diverse bursty impulse models have been proposed [80], [101], [102], [107], [109], [111], [112], [117], [120]. Specifically, as shown in Fig. 5b, both the background noise and the impulsive noise samples are modeled by considering them to occur in a burst, which spans over a number of consecutive time instants. Generally, bursty impulse modeling can be classified into temporally-independent and temporally-correlated scenarios, which are discussed as follows.

The noise impulses in the temporally-independent modeling scenarios can be represented by a gated Bernoulli process [112], consisting of impulse-free and impulse-impaired bursts alternatively, whose time durations are independent of each other. Assuming that the $l$-th burst spans over $t_{l}$ time intervals, the $l$-th noise burst can be expressed as [112]:

$$
\boldsymbol{n}_{l}=\boldsymbol{w}_{l}+b_{l} \boldsymbol{i}_{l}
$$

and

$$
t_{l}= \begin{cases}t_{d}, & \text { if } b_{l}=1 \\ t_{\mathrm{IAT}}, & \text { if } b_{l}=0\end{cases}
$$

where $b_{l}$ is a Bernoulli distributed random variable; the vectors of $\boldsymbol{w}_{l}$ and $\boldsymbol{i}_{l}$ consist of $t_{l}$ background and impulsive noise samples, respectively. Note that both $t_{d}$ and $t_{\mathrm{IAT}}$ are deterministic for periodic impulsive noise scenarios, while in non-periodic impulsive noise scenarios their values become random and may obey a specific distribution, e.g. the uniform [112], the exponential [117] and the log-normal [120] distributions.

In the temporally-correlated modeling, the duration of impulsive noise and the IAT are typically characterized using Markov chains. For example, Gilbert and Elliott [101], [102] modeled the noise process relying on a two-state Hidden Markov Model (HMM), where State $S_{1}$ is free from impulsive noise, while in State $S_{2}$ the noise sample contains impulsive noise. Let us define the transition probability from State $S_{i}$ at the $k$-th time instant to State $S_{j}$ at the $(k+1)$-th time instant as $p_{i j}=\operatorname{Pr}\left(s_{k+1}=S_{j} \mid s_{k}=S_{i}\right)$. As illustrated in Fig. 6, the transition between these two states can be characterized by a transition probability matrix, denoted by $\left(\begin{array}{ll}p_{11} & p_{12} \\ p_{21} & p_{22}\end{array}\right)$. The noise voltage of these two states obeys the zeromean Gaussian distributions associated with different variance

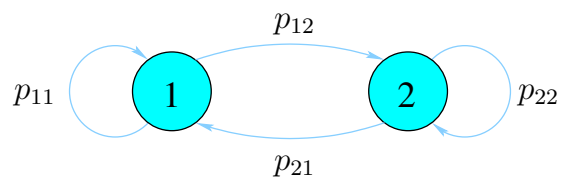

Fig. 6: Illustration of the Gilbert-Elliot model, which is a two-state Markov noise model, where State 1 refers to impulse-free instants while State 2 represents impulse-impaired instants. The transition probability $p_{i j}$ refers to the occurrence probability of State $j$, given the current state as State $i$.

values ${ }^{4}$. Since only two states are employed in this model, the associated small number of states is incapable of accurately characterizing a wide variety of noise process. To address this impediment, Ndo et al. [111] proposed a four-state HMM relying on the truncated Middleton's Class A model [105]. Beneficially, the impulsive noise is comprised of three components associated with different variance values. Furthermore, inspired by [40] and [111], Zhang et al. [117] modeled the noise using a two-state Hidden Semi-Markov model (HSMM), where the duration of the impulse-free states and the impulseinfested states obeys the exponential distribution associated with different mean values.

\section{Noise Mitigation Techniques}

In order to alleviate the deleterious effects imposed by impulsive noise, PLC systems have invoked sophisticated noise mitigation techniques both at the transmitter and at the receiver sides, detailed as follows.

The mitigation techniques employed at the transmitter side mainly include channel coding [53], [133], [136], [138], [153], [157], interleaving [132], [144], [158]-[161] and AutomaticRepeat-and-reQuest (ARQ) [130], [137], [139]. As regards to the mitigation techniques at the receiver side, they may be classified into parametric and non-parametric approaches [162], depending on the requirements concerning the noise's statistical knowledge. Parametric processing techniques used at the receiver side mainly include nonlinear processing [140], [146], [148], [150], [163]-[169], adaptive filtering [147], [149], [170]-[172], symbol detection [107], [111], [131], [145], [173]-[175], and iterative decoding [109], [134], [135], [143], [154], [163], [176]-[178]. When using parametric approaches, we assume that the noise obeys a particular statistical model and optimize the receiver based on the estimated parameters

\footnotetext{
${ }^{4}$ This Gilbert and Elliott model is a special case of the partitioned Markoy chains detailed in Section III-B5, where both $v$ and $w$ are set to 1 .
} 


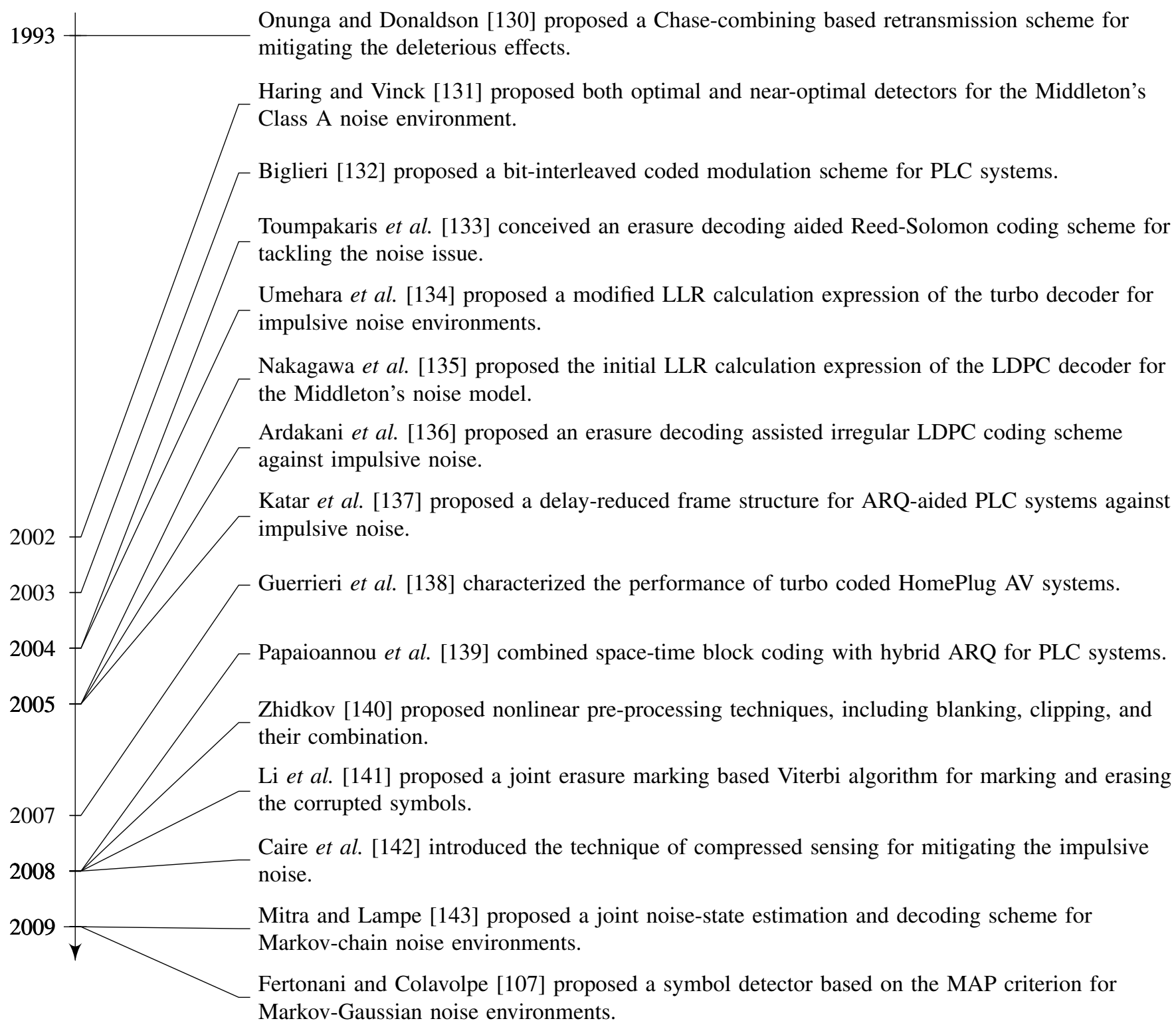

Fig. 7: Milestone papers on noise mitigation techniques (from the year of 1993 to the year of 2009).

of the model. By contrast, the statistical knowledge of noise process is not required when using non-parametric approaches [162]. Here we classify the non-parametric approaches into erasure decoding [133], [136], [141], [151], [157], [179]-[182] as well as compressed-sensing-aided mitigation techniques [142], [152], [155], [156], [162], [183]-[187]. The milestone papers on noise mitigation are summarized in Fig. 7 and Fig. 8. We continue to review the research advancements of these noise mitigation techniques in PLC individually as follows.

\section{A. Mitigation at Transmitter}

1) Channel Coding: In order to correct the errors caused by impulsive noise, numerous channel coding candidates have been considered [53], [133], [136], [138], [153], [157], ranging from maximum-minimum-distance Reed-Solomon codes [188] to the advanced turbo codes [189], LDPC codes [190] and Polar codes [191]. Specifically, Toumpakaris et al. proposed an erasure decoding aided Reed-Solomon coding scheme [133] for erasing the symbols corrupted by impulsive noise. Similarly, Ardakani et al. [136] proposed an erasure decoding assisted irregular LDPC coding scheme for an MC system in the presence of both additive white Gaussian noise and impulse noise. Hormis et al. [53] combined LDPC codes with cyclic random-error and burst-error correction codes for achieving a high degree of immunity of impulse noise. Guerrieri et al. [138] characterized the performance of turbo coded HomePlug AV systems, concluding that turbo codes are indeed capable of significantly improving its BER performance in the PLC channel suffering from narrow-band interference, especially when the decoder uses a sufficiently high number of iterations. Raptor codes, concatenating the Luby transform codes with LDPC codes, were shown to be capable of achieving nearcapacity performance [157]. As a further advance, Hadi et al. [153] investigated the performance of Polar-codes in the 


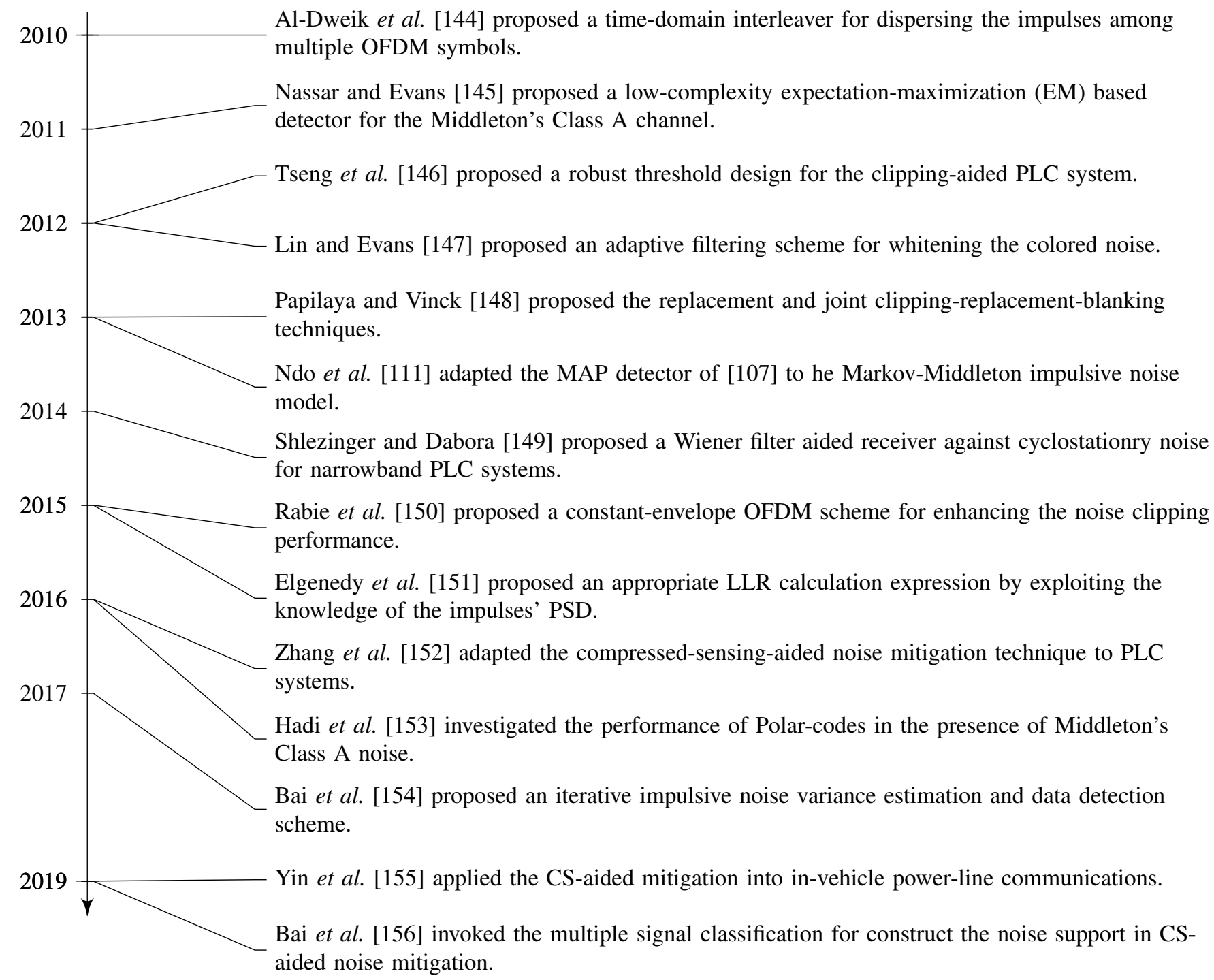

Fig. 8: Milestone papers on noise mitigation techniques (from the year of 2010 to the year of 2019).

presence of Middleton's Class A noise, where the simulation results also demonstrated that Polar codes outperformed LDPC codes in the face of Middleton's Class A noise.

Naturally, each channel coding scheme has a limited error correcting capability. However, the impulsive noise typically exhibits a bursty nature. In this case, the number of corrupted symbols may exceed the error correction capability of the channel coding scheme. This often leads to error propagation after decoding. This is because the excessive number of errors misleads the decoding process, which then amends the symbols in the wrong positions, hence resulting in avalanchelike error prorogation. Therefore, channel coding is usually invoked in combination with interleaving, as discussed below.

2) Interleaving: Interleaving is a well-known technique of randomizing the channel-induced errors by dispersing them. As shown in Fig. 9a, interleaving can be utilized in bitinterleaved coded modulation schemes for dispersing the error burst imposed by bursty impulsive noise [132], [144], [158][161]. Specifically, Caire et al. [158] presented the fundamental theory of bit-interleaved coded modulation. Biglieri
[132] mentioned that bit-interleaved coded modulation can be fruitfully exploited in PLC systems which are contaminated by impulsive noise. Nasri and Schober [159] conceived asymptotic analytical tools for conventional bit-interleaved coded modulation systems operating in non-Gaussian noise environments. Similarly, Nguyen and Bui [160] found the optimal soft-output demodulator of bit-interleaved coded modulation relying on iterative decoding in additive Class-A noise channels and analyzed its convergence behavior with the aid of the extrinsic information transfer chart. As a benefit, the number of errors in each decoding block is possibly below the error correcting capability and hence the avalanche-like error prorogation mentioned in Section IV-A1 can be avoided.

Furthermore, interleavers can be used at the symbol level for eliminating the error floors caused by impulsive noise. Specifically, once a time-domain sample is corrupted by an impulse in an OFDM symbol, the impulse is spread over the whole OFDM symbol after DFT-based demodulation and then the impulsive noise power becomes averaged over all subcarriers of the OFDM symbol. Since the impulsive noise 


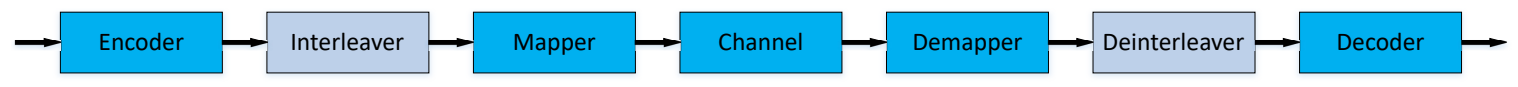

(a)

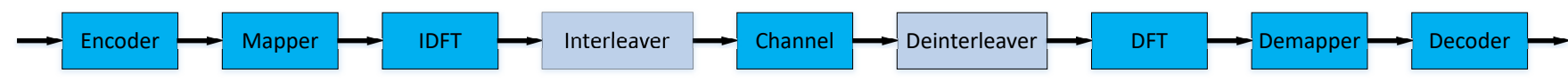

(b)

Fig. 9: Illustration of the interleaver in the face of impulsive noise: (a) block diagram of the bit-interleaved coded modulation scheme; (b) block diagram of the time-domain interleaver for OFDM systems.

usually occurs non-uniformly, the noise level of different OFDM symbols tends to fluctuate. This in turn results in an error floor, because the BER performance is predominantly determined by those OFDM symbols, which suffer from a high noise power. In order to mitigate the error floor, a time-domain interleaver (TDI) was proposed in [144]. As shown in Fig. 9b, its interleaver was placed after the inverse discrete Fourier transform (IDFT) based modulator while its deinterleaver was positioned before the DFT based demodulator. In this case, the impulses could not only be spread within an OFDM symbol but also among multiple OFDM symbols. As a benefit, the noise power after demodulation is expected to be maintained at a similar level and hence the error floors may be eliminated using a low transmit power. This TDI-based solution of [144] was further extended to time-domain interleaver with additional orthogonal transform (TDI-OT) in [161], where another IDFT-DFT block is inserted between the interleaver and deinterleaver of the TDI. Beneficially, the interleaver depth was quadratically increased, compared to that of [144], albeit at the cost of increasing the complexity. Note, however, that the BER performance improvement brought about by TDI and TDI-OT remains limited, when the noise pulses are nearuniformly distributed.

3) $A R Q: A R Q$ has been widely adopted in communications systems [130], [137], [139], as a benefit of its high robustness to sudden perturbations and of its low-complexity implementation. Specifically as shown in Fig. 10a, if a packet is correctly received at the receiver side [192], a positive acknowledgement (ACK) is sent back to the transmitter and then the next packet is transmitted. By contrast, if a corrupted packet is received, a negative ACK (NACK) is fed back to the transmitter and then the original packet is sent continuously, until the transmitter receives an ACK or the affordable number of retransmissions reaches its maximum limit.

Although it was originally designed for the MAC layer, ARQ can also be combined with channel coding in the physical layer, leading to the concept of hybrid ARQ (HARQ) [193], which can be classified into Chase-combining ${ }^{5}$ aided HARQ [194] and incremental-redundancy assisted HARQ [195]. As depicted in Fig. 10b, instead of being discarded, the previously received copies are jointly detected with the most recently received packet in the context of Chase-combining aided

\footnotetext{
${ }^{5}$ The original concept of the Chase-combining was proposed by David Chase in his 1985 IEEE TCOM paper entitled by "Code combining - a maximum-likelihood decoding approach for combining an arbitrary number of noisy packets". After that, the community has often used the fond conotaton of "Chase-combining" to refer to the "code-combining" for honoring his contribution.
}

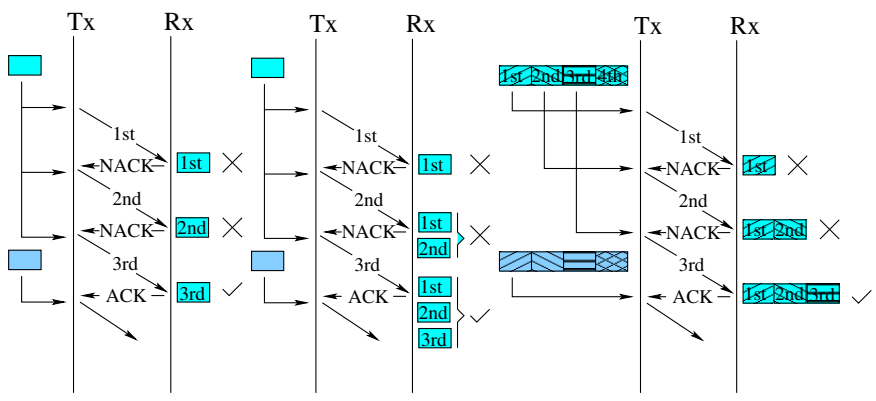

(a)

(b)

(c)

Fig. 10: Illustration of three types of HARQ, where 1st, 2nd and 3rd refer to the first, second and third transmission attempt, respectively. Furthermore, $X$ and $\checkmark$ represent the error detection event and no-error detection event, respectively. (a) ARQ; (b) Chase-combining HARQ; (c) Incremental-redundancy HARQ.

HARQ, while as regards to incremental-redundancy assisted HARQ, if a packet is corrupted, additional redundancy rather than the original packet is requested for joint decoding at the receiver as shown in Fig. 10c. The performance of the abovementioned ARQ schemes was compared in [196], revealing that the outage probability is the highest for the ARQ and the lowest for incremental-redundancy based HARQ.

Specific to the applications of ARQ in PLC, Onunga and Donaldson [130] proposed a Chase-combining based retransmission scheme, which was shown to be capable of efficiently mitigating the deleterious effects of impulsive noise. However, a disadvantage of ARQ techniques is their increased delay. To mitigate this impairment, Katar et al. [137] proposed a frame structure for ARQ-assisted PLC systems, where a transmission frame is encoded by a number of shorter FEC codewords, each of which has its own header information and can be checked by its own cyclic redundancy check. Using this scheme, we may localize the FEC codewords which are overwhelmed by impulsive noise and request a dedicated selective retransmission for them, instead of the whole transmission frame. As a benefit, the delay imposed by ARQ may be mitigated. Furthermore, since the time diversity is exploited by $A R Q$, naturally it can be jointly designed with the spacetime block coding, if a packet can be transmitted from multiple information sources. In light of this, Papaioannou et al. [139] proposed a cross-layer design for multicast PLC systems, where the space-time block coding was applied at the packet level instead of that at the symbol level. 


\section{B. Parametric Mitigation at Receiver}

1) Nonlinear Pre-processing at the Receiver's Input: As a benefit of its conceptual simplicity and ease of implementation, nonlinear pre-processing has been widely adopted in practice [140], [146], [148], [150], [163]-[169]. As shown in Fig. 11a, we use $y_{k}$ to denote the received signal at time instant $k$, whose amplitude and phase are processed by the nonlinear pre-processing unit. The output signal is denoted by $r_{k}$. Nonlinear pre-processing operation is carried out at the receiver's input and typically includes blanking [140], clipping [140], replacement [148] and their combination, for clearing up or for replacing the received signal corrupted by impulsive noise, which are detailed as follows. As for the low-complexity blanking technique shown in Fig. 11b, if the amplitude of $y_{k}$ exceeds the pre-set threshold denoted by $T_{b}, y_{k}$ is forced to zero-valued. Here denoting the output signal of the blanking technique at time instant $k$ by $r_{k}^{b}$, we have [140]:

$$
r_{k}^{b}= \begin{cases}y_{k}, & \text { if }\left|y_{k}\right| \leq T_{b} \\ 0, & \text { if }\left|y_{k}\right|>T_{b}\end{cases}
$$

In terms of the clipping technique [140] depicted in Fig. 11c, if the amplitude of $y_{k}$ exceeds the pre-set threshold denoted by $T_{c}, y_{k}$ is forced to $T_{c}$, while the phase remains unaltered. Here denoting the output signal of the clipping technique at time instant $k$ by $r_{k}^{c}$, we have [140]:

$$
r_{k}^{c}= \begin{cases}y_{k}, & \text { if }\left|y_{k}\right| \leq T_{c}, \\ T_{c} \exp \left(j \arg \left(y_{k}\right)\right), & \text { if }\left|y_{k}\right|>T_{c},\end{cases}
$$

where $\arg (x)$ refers to the argument of a complex number $x$. As plotted in Fig. 11d, blanking and clipping can also be combined in the form of a joint clipping and blanking function. Let us denote the output signal of the joint clipping and blanking technique at time instant $k$ as $r_{k}^{c b}$, which can be expressed as [140]:

$$
r_{k}^{c b}= \begin{cases}y_{k}, & \text { if }\left|y_{k}\right| \leq T_{c}, \\ r_{T}^{b} \exp \left(j \arg \left(y_{k}\right)\right), & \text { if } T_{c}<\left|y_{k}\right| \leq T_{b}, \\ 0, & \text { if }\left|y_{k}\right|>T_{b} .\end{cases}
$$

As an intermediate form of conventional clipping and blanking techniques, the so-called deep clipping shown in Fig. 11e, was applied to mitigate the destructive effects of impulsive noise in [168], which cuts the received signal linearly under a threshold $\beta T_{d c}$ and blanks the signal that exceeds the threshold. Here we denote the output signal of the deep clipping technique at time instant $k$ as $r_{k}^{d c}$ and we may formulate the function by [140]:

$$
r_{k}^{d c}= \begin{cases}y_{k}, & \text { if }\left|y_{k}\right| \leq T_{d c} \\ \left(T_{d c}-\alpha\right) e^{j \arg \left(y_{k}\right)}, & \text { if } T_{d c}<\left|y_{k}\right| \leq \beta T_{d c} \\ 0, & \text { if }\left|y_{k}\right|>\beta T_{d c}\end{cases}
$$

where we have $\alpha=\mu\left(\left|y_{k}\right|-T_{d c}\right) ; \mu$ is the clipping slope and $\beta=(1+\mu) / \mu$. Furthermore, Papilaya and Vinck [148] proposed an additional action termed as replacement as depicted in Fig. 11f, which replaced $y_{k}$ by the average magnitude of noiseless OFDM samples denoted by $|\bar{x}|$, if $y_{k}$ exceeded the replacement threshold $T_{r}$. Denoting the output signal of

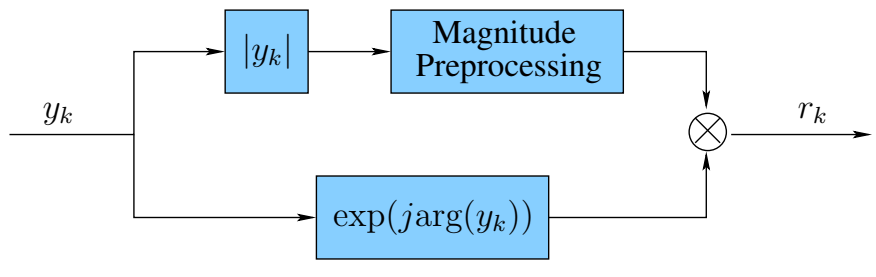

(a)

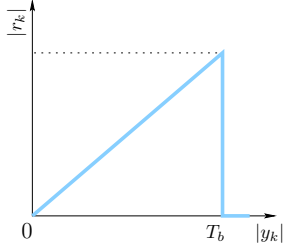

(b)

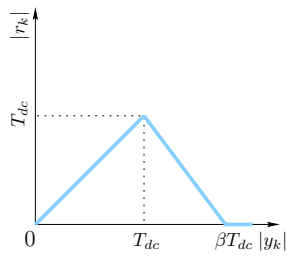

(e)

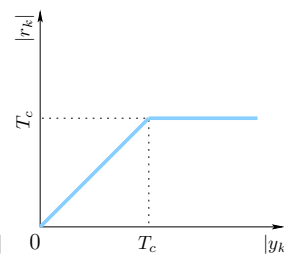

(c)

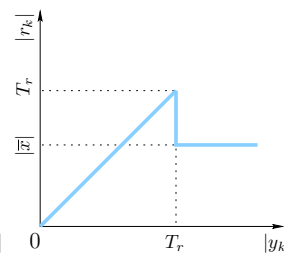

(f)

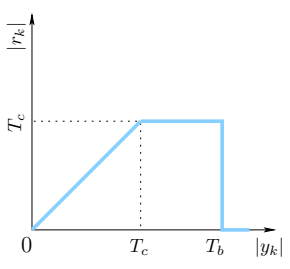

(d)

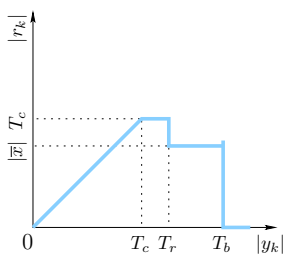

(g)
Fig. 11: Illustration of nonlinear pre-processing at the receiver's input. (a) Nonlinear pre-processing operation. (b) Blanking; (c) Clipping; (d) Clipping and blanking; (e) Deep clipping; (f) Replacement; (g) Clipping-replacementblanking.

the replacement technique at time instant $k$ by $r_{k}^{r}$, we may formulate the replacement function as:

$$
r_{k}^{r}= \begin{cases}y_{k}, & \text { if }\left|y_{k}\right| \leq T_{r}, \\ |\bar{x}| \exp \left(j \arg \left(y_{k}\right)\right), & \text { if }\left|y_{k}\right|>T_{r},\end{cases}
$$

where $|\bar{x}|=\sqrt{\left(\pi E_{s}\right) / 4}$ and $E_{s}$ is the average signal power per symbol. Replacement can also be inserted between the clipping and blanking stages, as seen in Fig. 11g. The corresponding clipping-replacement-blanking function is formulated as [148]:

$$
r_{k}^{c r b}= \begin{cases}y_{k}, & \text { if }\left|y_{k}\right| \leq T_{c}, \\ T_{c} \exp \left(j \arg \left(y_{k}\right)\right), & \text { if } T_{c}<\left|y_{k}\right| \leq T_{r}, \\ |\bar{x}| \exp \left(j \arg \left(y_{k}\right)\right), & \text { if } T_{r}<\left|y_{k}\right| \leq T_{b}, \\ 0, & \text { if }\left|y_{k}\right|>T_{b},\end{cases}
$$

where we use $r_{k}^{c r b}$ to denote the output signal of the clippingreplacement-blanking technique at time instant $k$.

The threshold used in nonlinear receiver pre-processing has to strike a trade-off between a high detection probability and low false alarm probability. In other words, useful signals should be preserved as much as possible, while the impulsive noise should be mitigated as best as possible. The threshold can be simply determined experimentally by finding the value associated with the minimum BER in simulation [165]. However, it requires long-term experiments and it remains only suitable for a specific impulsive noise scenario. Zhidkov [140] derived a closed-form SNR formula for the output signal of the nonlinear pre-processing unit, which can be used for 
threshold optimization by searching for the threshold value associated with the maximum SNR. Moreover, Ndo et al. [163] compared the threshold optimization performance between using the weighted combination criterion and using the Siegert criterion. These threshold optimization contributions in [140], [164] require a priori knowledge of the PDF of impulsive noise, which is usually difficult to obtain in practice. In order to address this issue, Tseng et al. [146] proposed an algorithm which is capable of determining the threshold relying on a rough range of the impulsive noise arrival probability at the receiver. Furthermore, Alsusa and Rabie [166] investigated the relationship between the optimal blanking threshold and the peak-to-average power ratio of OFDM systems and utilized it to determine the optimal threshold.

The disadvantages of nonlinear receiver pre-processing manifest themselves in at least two aspects. Nonlinear preprocessing causes inter-carrier interference in MC modulation. To overcome this impediment, Yih [197] proposed an iterative interference cancellation schemes. Mengi and Vinck [198] advocated a successive interference cancellation scheme in OFDM systems relying on clipping and blanking as well as on a syndrome decoder. Furthermore, the performance of nonlinear receive pre-processing is dependent both on the threshold selection and on the peak-to-average-power ratio (PAPR) of the transmitted signal. Specifically, given a sequence of signals transmitted using different transmit power, it is challenging to distinguish whether a signal is impaired by impulsive noise. Therefore, we have to employ the technique of the PAPR reduction at the transmitter side for ensuring the power of transmitted signals at a closely constant level. Particular to the MC modulation where high PAPR typically prevails, its reduction has attracted substantial attention [150], [167], [169], [199]-[204]. In general, PAPR reduction in MC modulation can be loosely categorized into signal clipping [201], peak cancellation [167], coding [199], tone reservation [200], selective mapping [169] and constant envelope design [150]. Specifically, Armstrong [201] proposed to impose hardlimiting on the signal received and then to pass it through a filter to reduce the out-of-band power. Juwono et al. [167] used a peak amplitude clipping block at the transmitter so that the clipper at the receiver may clip the "real" impulsive noise. David and Jedwab [199] encoded the transmitted signal using a Reed-Muller code and separated the codewords having high peak-to-mean envelope power ratio. Rabie and Alsusa [169] proposed to multiply each OFDM frame with a set of randomly generated phase vectors and the one associated with the lowest peak-to-average power ratio was then selected for transmission. Rabie et al. [150] placed an amplitude-phase modulator right after the OFDM modulator for ensuring that the peakto-average power ratio remained 1. Among these approaches, the technique of iterative clipping and filtering [202]-[204] is an appealingly low-complexity, yet efficient PAPR-reduction scheme. In this scheme, the signals are clipped using a predefined threshold and then a filter is employed for mitigating the out-of-band radiation. Given that the operation of filtering may result in a destructive signal, we may then clip and filter the results iteratively until the PAPR is sufficiently mitigated. Furthermore, some single carrier schemes were proposed for

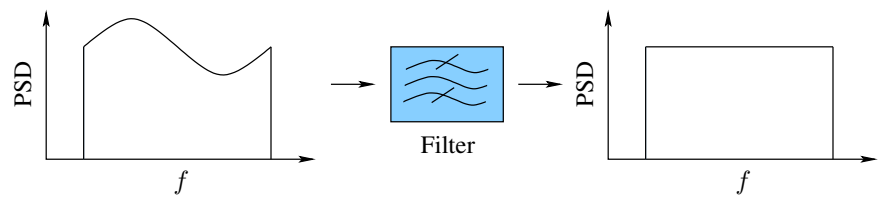

Fig. 12: Illustration of the adaptive filter designed for whitening the colored noise, where the PSD represents the power spectral density.

PLC systems as a benefit of their intrinsically lower PAPR, such as the single-carrier frequency-division multiple access scheme [205].

Finally, the afore-mentioned nonlinear pre-processing techniques can be integrated into other modules, e.g. TDI [112] and bit-loading [206], and may also be beneficially combined with channel coding [163], [178].

2) Adaptive Filtering: The noise components exhibiting slowly time-varying or deterministic spectral characteristics may be mitigated by invoking an adaptive filter. In general, the adaptive filter is used for equalization and prediction. As regards to equalization, as shown in Fig. 12, Lin and Evans [147] proposed a filtering scheme for whitening the colored noise, assuming the so-called LPTV noise model of [55]. As a further contribution, Yoo and Cho [170] proposed a linear minimum mean square error (MMSE) based method for estimating the parameters of correlated noise and designed a filter for equalizing the noise. As for the prediction filter based approach, García et al. [171] extracted the modeling parameters of the colored periodic noise using Yule-Walker methods and then designed a linear prediction filter for predicting and mitigating the noise effects. Similarly, Llano et al. [172] proposed a prediction filter for quasi-stationary noise and extracted the desired signal by subtracting the predicted noise from the received signal. Shlezinger and Dabora [149] proposed an adaptive frequency shift filter for exploiting the cyclostationary characteristics of the OFDM information signal as well as those of the narrow-band PLC noise. This scheme shows a substantial performance gain over those approaches only focusing on the information signal estimation.

3) Symbol Detection: Since conventional detectors are usually optimized for the ubiquitous AWGN environment, the non-Gaussian nature of impulsive noise in PLC degrades their optimality [145], [173]. To address this issue, Fukami et al. [174] designed a noncoherent frequency shift keying (FSK) detector for Middleton's Class A channel. Haring and Vinck [131] designed both optimum and near-optimum detectors for the Middleton's Class A noise environment and derived their general performance bounds. Nassar et al. [175] proposed a low-complexity expectation-maximization (EM) based detector for the Middleton's Class A channel. These three investigations were proposed for mitigating discontinuous Middleton's Class A noise. As regards to the bursty impulsive noise, the memory of the channel has to be taken into account. Specifically, Fertonani et al. [107] proposed a noise-mitigating symbol detection scheme for the channel contaminated by the Markov-Gaussian impulsive noise, by appropriately modifying the maximum a posteriori (MAP) criterion, which was capable of attaining the optimum performance. Similarly, Ndo et al. 
[111] adapted the optimum MAP detector of [107] to the Markov-Middleton impulsive noise model.

4) Iterative Decoding: Over the past decades, the design criteria of channel codes have evolved from conceiving maximum-minimum distance codes for Gaussian telephoneline channels to schemes suitable for fading channels. The errors imposed by wireless channels are no longer randomly distributed, which is in stark contrast to the uncorrelated error events of Gaussian channels, because the Rayleighfaded wireless channels inflict burst of errors. The duration and relative frequency of occurrence of these error burst depend on the Doppler-frequency of the channel, but they can be randomized with the aid of sufficiently long channel interleavers. By contrast, in PLC systems, the error bursts are typically much longer and their length depends on the duration of the impulse noise instances. At the time of writing, the most popular codes used for PLC systems are long LDPC codes and turbo codes relying on long turbo-interleavers and iterative decoding. Owing to having longer error-bursts in PLC than in fading wireless channels, the PLC systems tend to require longer interleavers. Another substantial difference with respect to Gaussian and Rayleigh channels is that the log-likelihood ratio (LLR) calculation required for iterative soft-decoding has to take into account the channel's statistics, as detailed in [207]. To solve this problem, Umehara et al. [134] modified the LLR calculation expression according to Middleton's Class A noise statistics, which enhanced the reliability of LLRs during the decoding iterations and hence significantly improved the BER performance compared to the conventional decoder. As regards to LDPC decoding, the formulas used for calculating the initial LLRs are valid for the sum-product decoding algorithm relying on the statistics of Middleton's Class A noise model [135]. As a further advance, upon assuming that the noise obeys a two-state MarkovGaussian model and assuming perfect knowledge of both the noise variance and of the state transition probabilities, Mitra and Lampe [143] proposed a joint iterative estimation and decoding algorithm. When considering the classic trellis-based decoding of convolutional codes, the expressions of the softmetric play a crucial role in predetermining the attainable performance. Specifically, their conventional soft-metric based on the Euclidean distance between the channel-contaminated received signal samples and the legitimate symbol values required no knowledge about the noise statistics, but sacrificed the performance compared to the more advanced metrics requiring more statistical knowledge about the noise. Accordingly, Mitra and Lampe [109] theoretically analyzed both the cut-off rate and the BER associated with various metrics in a Markov-Gaussian noise scenario for convolutionally coded systems.

Block codes have also been invoked for minimizing the effects of impulsive noise. For example, Haring and Vinck [176] proposed iterative decoding aided block codes for impulsive noise channels. More explicitly, the codes were optimized for spreading the noise impulses, which had a similar mitigating effects to that of the DFT block. The simulation results revealed that the errors caused by impulsive noise were substantially mitigated, leading to a performance close to that of the same scenario free from impulsive noise. Moreover, an RS coded OFDM system was proposed in [177].

Furthermore, iterative decoding can be invoked in combination with other processing techniques. For example, the technique of clipping was combined with LDPC codes [178] as well as turbo codes [163], respectively. Bai et al. [154] proposed an iterative impulsive noise variance estimation and data detection scheme, where the symbols at the output of the iterative decoder were compared to the received signals in order to improve the accuracy of impulsive noise variance estimation. Upon obtaining sufficiently accurate statistical characterization of the noise, we may feed reliable channel output LLRs to the data detection for achieving a reduced BER.

\section{Non-parametric Mitigation at Receiver}

1) Erasure Decoding: Both symbol detection and iterative decoding require the PDF of the noise process for noise mitigation. This is however difficult to obtain in practice. When the noise statistics are unknown, once a symbol is corrupted by impulsive noise, the corresponding LLRs obtained from the soft detector become unreliable due to the soft metric mismatch, and in case of iterative detection the errors may also be propagated to the symbols in the vicinity during the iterative decoding stage. A promising solution is to identify the low-confidence symbols impaired by impulsive noise and erase them during decoding. More explicitly, the large amplitude and bursty occurrence of impulsive noise allow us to spot the impaired symbols, which are then marked as erasures [179]. In this way, the LLRs corresponding to these symbols are not involved in the decoding process and hence the impulse-free symbols are protected from the noise impulses. This leads to an improved BER performance [133].

The research contributions on the erasure decoding techniques of copper-based communications can be classified into separate erasure and decoding [136], [151], [157] as well as joint erasure and decoding [141], [181], [182]. As regards to the separate erasure and decoding shown in Fig. 13a, the symbols that impaired by impulsive noise are marked as erasures before being fed into decoding block for correction. For example, Ardakani et al. [136] proposed a separate erasure and decoding scheme, where the corrupted symbols were detected using a distance-based detection technique [133], while their LDPC decoder was optimized for the erasure channel. As a further solution, Andreadou and Tonello proposed a concatenated coding scheme [157], where LDPC codes were employed as the inner code to identify the catastrophically error-infested packets, while Luby transform (LT) codes [180] were used as the outer code for correcting errors. Note that in this scheme the inner code and the outer code do not perform both erasure marking and decoding at the same time and hence the method of [157] belongs to the separate erasure and decoding. A disadvantage of the solution in [157] is that the erasure detection relies on the value of LLRs received from the soft demapper, while some large impulses may lead to large yet erroneous LLRs, which potentially result in wrong decisions. To overcome this issue, Elgenedy et al. [151] proposed a 


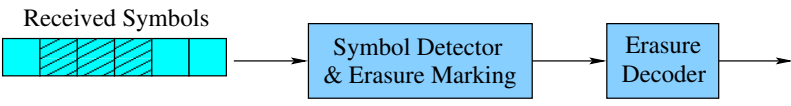

(a)

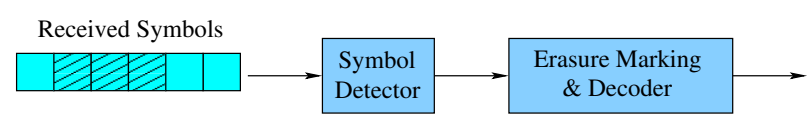

(b)

Fig. 13: Illustration of erasure decoding, where the shaded blocks refer to the symbols contaminated by impulsive noise. (a) Separate erasure and decoding; (b) Joint erasure and decoding.

technique for appropriately scaling the LLRs obtained upon weighting the PSD of impulses ${ }^{6}$, which improved the erasure detection reliability. By contrast, as shown in Fig. 13b, the joint erasure and decoding technique detects symbols first and then carries out both erasure and decoding within the same decoding block. For example, Li et al. [182] proposed a joint erasure marking based Viterbi algorithm (JEVA), where the decoding process was composed of two steps. Specifically, given a maximum number of $K$ symbols that can be erased, the first step is to determine the positions of erasures for the cases associated with $k \in\{0, \ldots, K\}$ erasures respectively, while the second step is to determine the minimum number of erasures. The schemes of [182] were later extended to the joint erasure marking and list Viterbi algorithm (JELVA) [141] by invoking the list Viterbi algorithm of [208] in order to improve the erasure marking accuracy.

2) Compressed-Sensing-Aided Mitigation: Training-based impulsive noise estimation relying on compressed sensing (CS) constitutes an attractive method, since it has several distinct advantages for OFDM-based PLC systems. Firstly, in practice, the high-attenuation frequency sub-bands of an OFDM symbol may be disabled for data transmissions [209], [210]. As a benefit, some of these deactivated tones can be used as training symbols for supporting training-based impulsive noise estimation. Secondly, it is possible to disperse the prolonged impulsive bursts affecting numerous timedomain samples by simply using an interleaver. In this way, the asynchronous impulsive noise can be estimated at a low complexity with the aid of CS, as shown in Fig. 14. Finally, since the power of asynchronous impulsive noise is usually much higher than that of the background noise, accurate impulsive noise estimation becomes attainable by using CS.

The idea of applying CS to mitigate the impulse noise in OFDM systems was originally proposed in [142], where the impulse noise estimation was formulated as an $\ell_{1}$ minimization problem. Later in [183], the mixed $\ell_{2} / \ell_{1}$ minimization has been employed for impulse noise estimation, where the impulsive noise was assumed to appear in form of sparse blocks. It should be noted that in [142] and [183] the duration of impulse noise was assumed to be much lower than that of an OFDM symbol, which is however, not the norm. Furthermore, although the $\ell_{1}$-minimization considered in [142] and the mixed $\ell_{2} / \ell_{1}$-minimization of [183] can be

\footnotetext{
${ }^{6}$ Since the PSD of impulsive noise has to be estimated in this scheme, it should be classified as a parametric method under the strict definition.
}

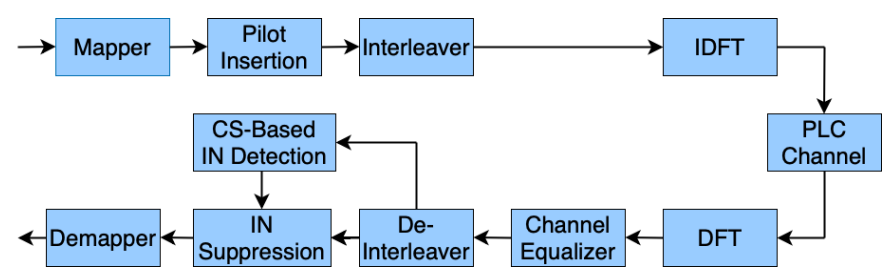

Fig. 14: Illustration of compressed-sensing-aided mitigation in PLC systems contaminated by impulsive noise, where IN represents impulsive noise.

carried out within polynomial rather than exponential time, the corresponding computational cost still remains excessive. It is important to emphasize that for both schemes substantial computational resources are required for estimating low-level impulsive noise, whose instantaneous power is lower than the modulation-dependent detection threshold. In [184], the so-called basis pursuit denoising technique relying on adaptive threshold detection was applied for coarsely estimating impulsive noise samples. The authors of [162] proposed a sparse Bayesian learning approach for mitigating impulsive noise, demonstrating that as expected, the performance can be improved upon increasing the number of pilot symbols in OFDM systems. In [185], an a priori-aided matching pursuit approach was proposed for mitigating the impulsive noise, where the time-domain support of the impulsive noise process was assumed to be partially known. A novel compressed impairment sensing assisted and Interleaved-DoubleFFT (IDFFT) aided system was proposed [152], which has been shown to be capable of simultaneously mitigating both the multipath effects and the impulsive noise impairments. Korki et al. [186] proposed a block-based iterative Bayesian algorithm for mitigating the deleterious effects of impulse bursts at the receiver side without using interleaving. In this scheme, the impulsive noise samples are divided into several blocks, so that existing block sparse recovery algorithms can be used for reconstructing the impulsive noise. Although the delay caused by the interleaving operation is avoided by this approach, the corresponding signal processing delay may increase. Given that the narrow-band interference exhibits slowly time-varying spectral behavior, Liu et al. [187] proposed a compressed sensing aided interference mitigation scheme for PLC systems, where the temporal correlation of the narrow-band interference is inferred from the temporal differences that are assumed to be known. Recently, Yin et al. [155] applied the CSaided mitigation into in-vehicle power-line communications. The technique of multiple signal classification was applied for construct the support of the impulsive noise and the impulsive noise was recovered with the aid of sparse Bayesian learning algorithm [156].

Remark 2. Table IV compares these impulsive noise mitigation techniques from the perspective of the target noise type, the mitigation performance, as well as the spectral efficiency attained, the computational complexity imposed, and the processing delay. It can be readily seen that nonlinear preprocessing, coding, and CS-aided mitigation techniques are the most popular techniques. It is plausible that the various PLC system characteristics of Table IV tend to require a 
TABLE IV: Comparison of impulsive noise processing techniques, where Tx/Rx refers to the transmitter or the receiver; CS means compressed sensing. A larger number of stars implies a larger value of that metric.

\begin{tabular}{|c|c|c|c|c|c|c|}
\hline $\mathrm{Tx} / \mathrm{Rx}$ & $\begin{array}{l}\text { Processing } \\
\text { Techniques }\end{array}$ & $\begin{array}{r}\text { Type of } \\
\text { Noise }\end{array}$ & $\begin{array}{r}\text { Mitigation } \\
\text { Performance }\end{array}$ & $\begin{array}{r}\text { Spectral } \\
\text { Efficiency }\end{array}$ & $\begin{array}{r}\text { Computational } \\
\text { Complexity }\end{array}$ & $\begin{array}{r}\text { Processing } \\
\text { Delay }\end{array}$ \\
\hline \multirow{3}{*}{ Transmitter } & $\begin{array}{l}\text { Channel Coding [53], [133], [136], } \\
{[138],[153],[157]}\end{array}$ & Discontinuous & 弐文 & 勾方公 & 弐地 & 弐场 \\
\hline & $\begin{array}{l}\text { Interleaving [132], [144], [156], [158]- } \\
{[161]}\end{array}$ & Consecutive & 弐次 & 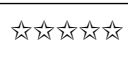 & 弐地 & 约次方 \\
\hline & $\begin{array}{l}\text { Automatic-repeat-request } \\
{[130],[137],[139]}\end{array}$ & Any & 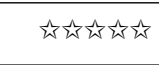 & 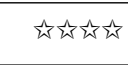 & 放 & 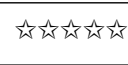 \\
\hline \multirow{4}{*}{$\begin{array}{l}\text { Parametric } \\
\text { Receiver }\end{array}$} & $\begin{array}{l}\text { Nonlinear Preprocessing [140], [146], } \\
{[148],[150],[163]-[169]}\end{array}$ & Any & 弐 & 地地方 & 放 & 头 \\
\hline & $\begin{array}{l}\text { Adaptive Filtering [147], [149], [170]- } \\
{[172]}\end{array}$ & Colored & 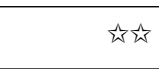 & 地地方 & 弐地 & 弐方 \\
\hline & $\begin{array}{l}\text { Symbol Detection [107], [111], [131], } \\
{[145],[173]-[175]}\end{array}$ & Any & 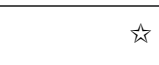 & 它地方 & 弐次 & 弐方 \\
\hline & $\begin{array}{l}\text { Iterative Decoding [109], [134], [135], } \\
{[143],[154],[163],[176]-[178]}\end{array}$ & Discontinuous & 弐第 & 勾方公 & 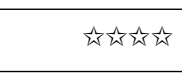 & 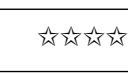 \\
\hline \multirow{2}{*}{$\begin{array}{l}\text { Nonparametric } \\
\text { Receiver }\end{array}$} & $\begin{array}{l}\text { Erasure Decoding [133], [136], [141], } \\
{[151],[157],[179]-[182]}\end{array}$ & Any & 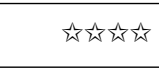 & 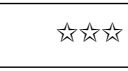 & 弐施地 & 在公公 \\
\hline & $\begin{array}{l}\text { CS-aided mitigation [142], [152], } \\
{[155],[156],[162],[183]-[187]}\end{array}$ & Discontinuous & 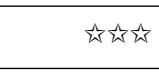 & 弐方公公 & 弐方出 & 级定公 \\
\hline
\end{tabular}

compromise. To elaborate, we can always improve the performance by invoking more complex signal processing techniques. However, this often imposes a higher delay, for example due to using longer FEC-decoding interleavers and/or more $A R Q$ retransmission attempts. Naturally, the above characteristics also depend on the specific type of impairments to be mitigated. Future system design should ideally aim for finding all the optimal operating points of PLC systems, where none of the above-mentioned characteristics can be improved without degrading at least one, or even several of these features. The collection of these optimal operating points constitutes a socalled Pareto-front in multi-component optimization. Hence, future high-impact frontier-research may be expected to solve the open problem of populating the optimal Pareto-font with compelling practical solutions.

\section{Joint Noise Mitigation And Channel Estimation}

As mentioned in Section II-A, the PLC channel exhibits a time-variant nature, caused by non-linear devices in appliances and by plug-in/out actions. Hence channel estimation (CE) has to be employed in PLC systems. However, the occurrence of impulsive noise degrades its accuracy, which may lead to erroneous detection and error propagation throughout the consecutive signal processing stages. To address this issue, there have been a range of insightful contributions in the literature [211]-[216], which are summarized in Fig. 15 and introduced from the perspectives of pilot insertion and iterative estimation as follows.

Since the PLC channel exhibits correlation, which manifests itself both in the time- and in the frequency-domain, it is beneficial to estimate the channel by inserting frequency-domain (FD) pilots, which constitute a small fraction of the transmitted symbols. Specifically, pilot-aided $C E$ can be carried out in three steps. Firstly, FD pilots are inserted into the subcarriers of the transmitted OFDM symbols. Secondly, upon receiving the pre-defined pilot sub-carriers, the FD channel response corresponding to the known subcarriers can be directly obtained. Thirdly, the channel responses of the subcarriers between two consecutive FD pilots can be found by interpolation. For example, Rinne et al. proposed a joint impulse burst position detection and channel estimation algorithm [211]. To elaborate a little further, three types of pilots insertion were considered. Moreover, an impulse burst was deemed to be detected, when the received pilots exceeded a pre-set threshold, while the channel was estimated using the classic linear minimum mean square error (LMMSE) estimator. The disadvantage of this pilot-aided CE method is its reduced spectral efficiency. To overcome this shortcoming, CS relying on null subcarriers has emerged in impulsive noise environments. For example, Mehboob et al. [213] proposed a joint channel and impulsive noise estimation for OFDM-based PLC systems relying on CS, where a single set of pilots was used for both channel estimation and impulsive noise reconstruction. As a benefit, the number of pilots employed was reduced.

The motivation of iterative $C E$ is to improve the estimation accuracy by exploiting either the soft information of the data symbols or the symbols recovered from impulsive noise. As for the soft information, it can be obtained from the decoder's output. Nassar et al. proposed a factor graph approach for joint channel estimation and decoding in PLC [214]. Assuming that the noise is modeled as the Gaussian mixture and the Bernoulli-Gaussian hidden Markov model, the authors modified the a posteriori probability formula, which was used during the iterations between the $\mathrm{CE}$ and the decoding. The iterations terminate when an accurate $\mathrm{CE}$ result is attained. In terms of the $\mathrm{CE}$ using the recovered symbols, pilots or training symbols are used for estimating the initial channel response, which facilitates the mitigation of the impulsive noise. Then the recovered symbols are fed back to re-estimate the channel response for improving the CE accuracy. For example, Chien proposed an iterative $\mathrm{CE}$ and impulsive noise mitigation approach in [215]. Specifically, the impulsive noise was firstly mitigated using nonlinear pre-processing and then channel responses were estimated according to the initially recovered symbols. Following this, the impulsive noise was mitigated using post-processing and the newly updated symbols were 


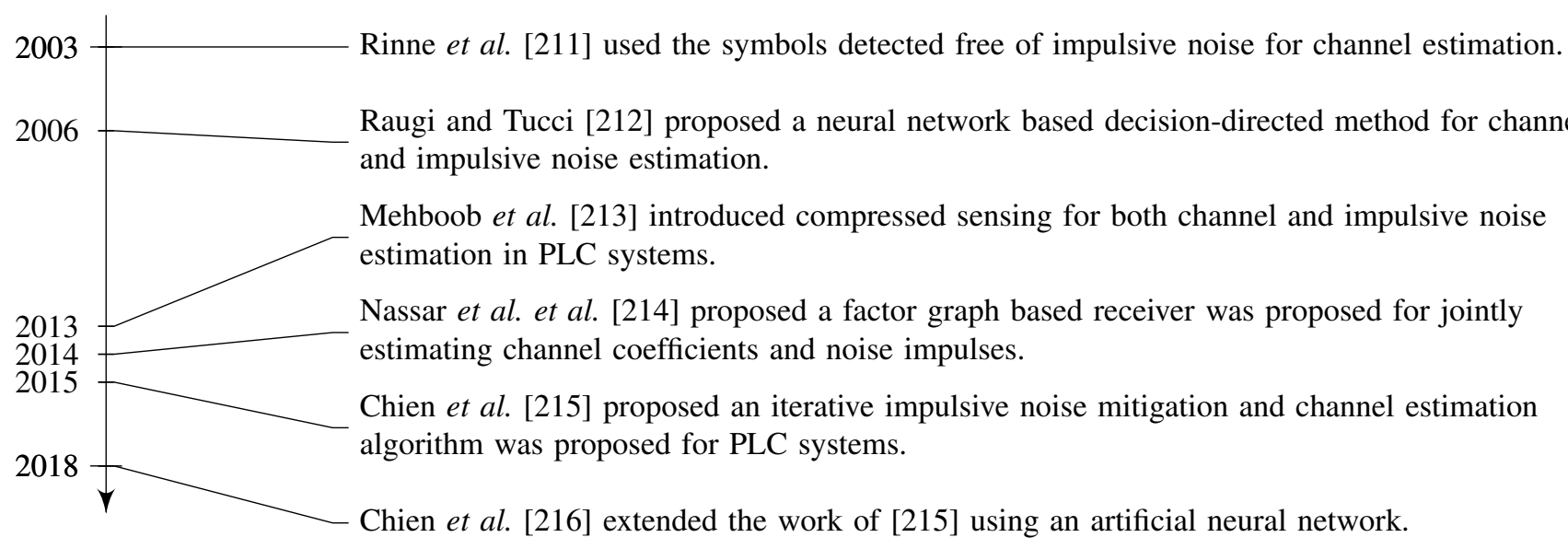

Fig. 15: Milestone papers on joint noise mitigation and channel estimation.

then fed back for improving the CE accuracy. The method in [215] was further extended using an artificial neural network in [216], which was capable of improving both the impulsive noise detection and the estimation accuracy.

\section{Future RESEARCH DiRECTIONS AND EXTENSIONS}

In this section, we highlight a number of challenges and the corresponding future research directions from the perspectives of both noise modeling and of noise mitigation. Furthermore, since noise is also deemed to be a crucial issue in some other communications systems, the extensions of techniques surveyed to other domains are also discussed.

\section{A. Future Research Direction of Noise Modeling}

1) From SISO PLC to MIMO PLC: Inspired by the family of multiple-antenna aided systems in wireless communications, MIMO PLC relying on the live, neutral and protective earth wires have attracted substantial attention [83] for boosting the capacity of the PLC transmission in the era of Gbps communications [24]. Particular to the noise in MIMO PLC, apart from the temporal and spectral correlations detailed in Section III, spatial correlation is also observed among the noise process in those three wires [217], which imposes a new challenge on the noise modeling. There have been some insightful research contributions [69], [218], [219] on investigating the noise-resilience in MIMO-aided PLC. Specifically, Hashmat et al. [69] proposed a time-domain model of background noise for in-home MIMO PLC systems; Elgenedy et al. [218] provided a model for the family of cyclostationary noise processes contaminating MIMO NB-PLC; the interference processes of MIMO PLC are characterized in [219]. However, the non-periodic impulsive noise has not been hitherto characterized. Furthermore, the distinct lack of analytic formulas of accurate noise modeling hampers the further performance analysis and system design. Therefore, a complete model of the holistic noise environments of MIMO PLC has to be proposed.

2) From Consumer Applications to Industrial Applications: The existing research contributions mainly focus their attention on modeling the noise in consumer applications, namely on the scenarios of energy distribution management in smart grids and on in-home broadband network access. As a benefit of the ubiquitous electric power infrastructure, PLC also has a potential of supporting various industrial scenarios, e.g. energy transmission management [220] and monitoring [221] in smart grids, intelligent transportation [222], factory automation [223], as well as Internet of Things [224]. Since the topology of the electric wires in industrial scenarios is drastically different from that in consumer applications and some new noise sources are introduced, the previously established noise models may not retain their accuracy in industrial scenarios. Furthermore, the mission-critical applications found in industrial scenarios typically impose stringent requirements on the communications reliability. Therefore, it becomes vitally important to evaluate the noise behavior in industrial scenarios.

3) From Noise Characterization to Noise Emulation: The ultimate role of noise characterization is to assist in designing sophisticated PLC systems, which have to be tested in diverse operational scenarios. This involves tremendous efforts and costs. As an intermediate solution, the testing can be initially conducted by relying on a noise emulator. The challenges of designing a reliable noise emulator manifest themselves in at least three aspects. Firstly, the various noise components should be extracted from the raw measurement data and then parameterized. Secondly, accurate models should be selected for the different noise components. Thirdly, a noise emulator should be implemented for synthesizing real-time noise sequences. Han et al. [79] proposed a synthetic noise emulator for SISO PLC systems operating at the frequency band spanning from $0.15 \mathrm{MHz}$ to $10 \mathrm{MHz}$. However, new noise emulators have to be proposed for broader frequency bands as well as for MIMO-PLC operating in industrial scenarios.

\section{B. Future Research Direction of Noise Mitigation}

1) From Single-Source Mitigation to Hybrid-Source Mitigation: The existing research contributions mainly focus their attention on the investigation of a single noise mitigation technique. In practical PLC environments, however, diverse 
noise sources co-exist. Hence the system is expected to employ hybrid noise mitigation techniques. Unfortunately, a specific mitigation technique may reduce the efficiency of another one. For example, the symbol detection which was originally optimized according to the statistical knowledge of the noise process, may lose track of the channel's memory if simultaneously nonlinear pre-processing is invoked at the receiver's input, because this nonlinear pre-processing unit may reshape the statistics of the original noise process. Therefore, how to jointly design multiple impulsive noise mitigation techniques remains an open research question for further investigation.

2) From Unified Noise Mitigation to Application-Specific Noise Mitigation: Given the diverse variety of application scenarios of PLC, a unified noise mitigation scheme is incapable of meeting all their requirements. Therefore, applicationspecific mitigation schemes should be proposed. Two facts have to be carefully considered when we design an applicationspecific mitigation scheme. One is that the knowledge of noise characteristics decides upon the particular selection of parametric or non-parametric processing at the receiver. The other one is that different applications impose diverse qualityof-service requirements on PLC systems. For example, inhome broadband network access exhibits stringent spectralefficiency specifications, but loose delay requirement, whereas the PLC systems used for supporting controller area networks are expected to accommodate both delay and reliability requirements. As seen in Table IV, each technique has its strengths and weaknesses in terms of noise mitigation efficiency, computational complexity, processing delay and spectral efficiency. In this case, how to match the advantages of the techniques to the application requirements remains an open issue, calling for the systematic exploration of the entire Pareto font.

3) From Model-Based Noise Mitigation to Data-Driven Noise Mitigation: The signal processing techniques applied in communications systems have solid statistical and information theoretical foundations, which are often accompanied by tractable mathematical models. Furthermore, they usually obey linear, stationary and Gaussian statistics. By contrast, the occurrence of impulsive noise in PLC introduces intractable non-stationary factors. Hence, a machine-learning (ML) based communications system that does not require a tractable mathematical model may be capable of improving the attainable performance [225]. On one hand, ML-based methods can be utilized for augmenting parts of the existing algorithms. For example, the generation of impulsive noise in the home is often related to the human behavior of switching on/off home appliances and other electronic devices. In this context, ML-based algorithms can be used for investigating the human behavior and accordingly predicting the arrival of impulses. As a benefit, both the spectral efficiency and the processing delay may potentially be improved, if the impulsive noise mitigation can be triggered only when impulses are indeed predicted to occur. On the other hand, inspired by the concept of "autoencoder" [226], we may directly apply ML to the physical layer, by completely replacing the existing communications systems. To elaborate, the chain of multiple independent blocks (channel codec, modem, etc.) can be replaced by a single deep-learning (DL)-based black box. Beneficially, we may improve the performance by exploiting the joint optimization capability of such a DL-based black box. Furthermore, the instantaneous knowledge of both the channel and of the noise is no longer a prerequisite. Hence, the corresponding overheads can be avoided ${ }^{7}$, resulting in an enhanced spectral efficiency. Indeed, the rather challenging Pareto-optimization may also be facilitated by powerful ML algorithms relying on the "auto-encoder" principle.

\section{Extensions to Other Communications Areas}

Apart from PLC systems, noise has also been a crucial issue in some other communications systems, including digital subscriber lines, underwater acoustic communications, and wireless communications. Compared to those in other communications systems, the noise process of PLC exhibits the most complex behavior and has attracted substantial research attention. Hence, the noise modeling and mitigation techniques surveyed in this article may also play an inspirational role in other domains, as detailed below.

1) Digital Subscriber Lines: Noise in digital subscriber lines typically comprises of background noise and impulsive noise [227]. Impulses in digital subscriber lines are mainly induced by switch-on/off of home appliances and by radio frequency interference, and by FM radio broadcasting. Similar to the impulses in PLC, the impulses in digital subscriber lines are classified into repetitive electrical impulse noise, single high impulse noise events and prolonged electrical impulse noise [228]. Specifically, the repetitive electrical impulse noise may be modeled by the techniques proposed for mitigating periodic impulsive noise, as detailed in Section III-B3 and Section III-B4. By contrast, both the single high impulse noise and prolong electrical impulse noise events can be modeled using the techniques originally conceived for non-periodic asynchronous impulsive noise, as detailed in Section III-B5. Furthermore, the Bernoulli-Gaussian Model [229] and the temporal-correlated model [154] detailed in Section III-C may be used for lending themselves to convenient mathematical analysis and to system design. As for noise mitigation, the current standard, namely XG-Fast [230], has already exploited the classic techniques of channel coding, of interleaving, of ARQ, and of erasure decoding, while both symbol detection and iterative decoding as well as learning-aided noise mitigation have been considered in the next-generation standard, namely Terabit DSL [231].

2) Underwater Acoustic Communications: The impulsive noise in underwater acoustic communications systems is typically caused by natural sources (including bio-acoustic sounds, water agitation and crustal movement) and human activities (for example, shipping, oil and gas exploration and production) [232]. Among these noise components, the snapping shrimp noise plays a dominant role [233]. The noise process is usually modeled as a stationary $\alpha$-sub-Gaussian noise associated with a memory order $m$ [233], which is essentially an impulsive Markov process of order $m$ and can be understood with the

\footnotetext{
${ }^{7}$ The benefit becomes distinct, especially when the "auto-encoder" is trained in an offline manner.
} 
aid of the discussions in Section III-C2. In order to simplify the performance analysis and system design, simple mathematical models, such as the Gaussian Mixture model detailed in Section III-C1, have also been considered in noise modeling [234]. The state-of-the-art in noise mitigation in underwater acoustic communications can be classified into nonlinear preprocessing [232], symbol detection [233], and compressedsensing-aided noise mitigation [235]. More advanced hybrid noise mitigation relying on combining the techniques detailed in Section IV is capable of further enhancing the robustness of underwater acoustic systems against impulsive noise. Furthermore, since the underwater acoustic channels vary over time, the joint channel and impulsive noise estimation detailed in Section V may also find application in this scenario. Having said that, the high Doppler frequency associated with the low operational frequencies of the acoustic signal remain a critical research issue [236].

3) Wireless Communications: Noise sources in wireless communications are quite diverse. For example, impulsive noise in office and retail environments is mainly caused by printers, elevators and microwave ovens [86]. Impulsive noise in industrial environments is usually induced by motors, heavy machinery, ignition systems, voltage regulators, welding equipment and electric switch contacts [237]. Vehicular communications is vulnerable both to impulsive noise caused by ignition and narrow-band interference [238]. The wireless sensor networks within power substations have to also operate in the face of the impulsive noise created by high-voltage equipment [73]. In particular, the empirical model based on the measurement results of [86] adopted similar techniques to those detailed in Section III-B, while the Bernoulli-Gaussian model, the Middleton's Class A model and the partitioned Markov chain model were adopted in [141], [238], and [73], respectively, which can be understood with the aid of Section III-C1 and Section III-B5. A number of research contributions have been proposed for tackling the noise issue in wireless communications, including erasure decoding [141], nonlinear pre-processing [239], compressedsensing-aided noise mitigation [238], and sophisticated symbol detection [240], which have been covered in Section IV. The optimal Pareto-font collecting all optimal solutions may be conceived with the aid of Table IV in future research.

\section{CONCLUSIONS}

In this paper, we commenced with a rudimentary introduction to PLC applications and then briefly presented the PLC channel characteristics and modulation schemes. The research contributions on noise modeling and on their mitigation over the past fifty years were reviewed. The key lessons learned are summarized as follows.

- Owing to its ubiquitous presence, PLC has been widely applied in diverse industrial and consumer scenarios, including the smart grid, smart home, smart factories, intelligent transportation and broadband network services. Despite these research advances, information theory indicates that the capacity of PLC has not been fully exploited [24], [241], predominantly due to the deleterious effects of impulsive noise.
- In contrast to the Brownian motion of electrons, which results in AWGN, the noise in PLC comprises of diverse noise components, including colored background noise, three types of impulsive noise, and narrow-band interference. This complex behavior severely degrades the integrity of PLC.

- Noise can be modeled based on empirical measurement campaign and on mathematical derivations. The empirical models are capable of accurately reflecting the characteristics of noise, but they are not friendly to performance analysis or to system design, because often intractable functions are involved in modeling. This issue can be tackled by using simplified mathematical models, whose parameters may be found from the empirical campaigns for accurately reflecting their practical characteristics.

- Noise mitigation techniques may be employed both at the transmitter side and at the receiver sides. The mitigation techniques applied at the transmitter side mainly include channel coding, interleaving and ARQ. The mitigation techniques used at the receiver side may be further classified into parametric and non-parametric approaches. Parametric processing techniques, including nonlinear processing, adaptive filtering, symbol detection, and iterative decoding, require the statistical knowledge of noise, while the non-parametric approaches, including erasure decoding as well as compressed-sensing-aided mitigation techniques, may perform well even without the noise's statistical information. The performance can always be improved by invoking more complex signal processing techniques. Yet, this often imposes a higher delay. Naturally, the above characteristics also depend on the type of impairments to be mitigated. Future system design should ideally aim for finding all the optimal operating points of PLC systems.

- In order to accommodate the noise environment of wider application scenarios of PLC, the noise characteristics of both MIMO PLC systems and of diverse industrial applications have to be accurately modeled. Following this, a noise emulation platform capable of accurately reflecting the noise characteristics can be constructed. Furthermore, as highlighted in Section VI-B, a range of techniques relying on hybrid-source mitigation, on application-specific noise mitigation and on data-driven noise mitigation can be conceived by future research for approaching the capacity of PLC. Finally, the family of noise modeling and mitigation techniques surveyed in this article may also find its way into other communications areas, including digital subscriber lines, underwater acoustic communications and wireless communications.

In summary, the potential of PLC has not been fully exploited and there remains a substantial room for improvement in the context of its noise modeling and mitigation, before it theoretical capacity is approached.

\section{REFERENCES}

[1] L. Lampe, Power Line Communications: Principles, Standards and Applications from multimedia to smart grid. John Wiley \& Sons, 2016. 
[2] K. Dostert, "Telecommunications over the power distribution gridpossibilities and limitations," IIR-Powerline, vol. 6, p. 97, 1997.

[3] N. Pavlidou, A. J. H. Vinck, J. Yazdani, and B. Honary, "Power line communications: state of the art and future trends," IEEE Commun. Mag., vol. 41, no. 4, pp. 34-40, April 2003.

[4] M. Schwartz, "History of communications: carrier-wave telephony over power lines: early history," IEEE Commun. Mag., vol. 47, no. 1, pp. 14-18, Jan. 2009.

[5] H. C. Ferreira, H. M. Grové, O. Hooijen, and A. Han Vinck, Power line communication. Wiley Online Library, 2010.

[6] H. C. Ferreira, L. Lampe, J. Newbury, and T. G. Swart, Power line communications: theory and applications for narrowband and broadband communications over power lines. John Wiley \& Sons, 2011.

[7] P. Van Der Gracht and R. Donaldson, "Communication using pseudonoise modulation on electric power distribution circuits," IEEE Trans. Commun., vol. 33, no. 9, pp. 964-974, Sept. 1985.

[8] G. Bumiller, L. Lampe, and H. Hrasnica, "Power line communication networks for large-scale control and automation systems," IEEE Commun. Mag., vol. 48, no. 4, pp. 106-113, April 2010.

[9] S. T. Mak and D. L. Reed, "TWACS, a new viable two-way automatic communication system for distribution networks. Part I: Outbound communication," IEEE Trans. Power Apparatus Syst., vol. PAS-101, no. 8, pp. 2941-2949, 1982.

[10] D. Clark, "Powerline communications: Finally ready for prime time?" IEEE Internet Computing, vol. 2, no. 1, pp. 10-11, 1998.

[11] S. Galli, A. Scaglione, and Z. Wang, "For the grid and through the grid: The role of power line communications in the smart grid," Proc. IEEE, vol. 99, no. 6, pp. 998-1027, June 2011.

[12] W. de Villiers, J. H. Cloete, L. M. Wedepohl, and A. Burger, "Realtime SAG monitoring system for high-voltage overhead transmission lines based on power-line carrier signal behavior," IEEE Trans. Power Del., vol. 23, no. 1, pp. 389-395, Jan. 2008.

[13] Y. Huo, G. Prasad, L. Atanackovic, L. Lampe, and V. C. Leung, "Grid surveillance and diagnostics using power line communications," in Proc. IEEE Int. Symp. Power Line Commun. Appl. (ISPLC 2018), Manchester, UK, April 2018, pp. 1-6.

[14] S. P. Dash, S. Joshi, and R. K. Mallik, "Smart grid network with D2D communication and coherent PLC: Error analysis," IEEE Trans. Veh. Technol., vol. 69, no. 1, pp. 1051-1054, Jan. 2020.

[15] H. Krattenmacher and R. Hien, "Single line network installation (SNI) - a new installation topology based on power line communication technology," in Proc. IEEE Int. Conf. Netw. Sensing Syst. (INSS 2010), Kassel, Germany, June 2010, pp. 30-32.

[16] S. C. Pereira, A. S. Caporali, and I. R. Casella, "Power line communication technology in industrial networks," in Proc. IEEE Int. Symp. Power Line Commun. Appl. (ISPLC 2015), Austin, TX, USA, April 2015, pp. 216-221.

[17] T. Sauter and M. Lobashov, "End-to-end communication architecture for smart grids," IEEE Trans. Ind. Electron., vol. 58, no. 4, pp. 1218 1228, April 2011.

[18] A. Sittoni, D. Brunelli, D. Macii, P. Tosato, and D. Petri, "Street lighting in smart cities: A simulation tool for the design of systems based on narrowband PLC," in Proc. IEEE Int. Smart Cities Conf. (ISC2), Guadalajara, Mexico, Oct. 2015, pp. 1-6.

[19] A. Pittolo, M. D. Piante, F. Versolatto, and A. M. Tonello, "In-vehicle power line communication: Differences and similarities among the incar and the in-ship scenarios," IEEE Veh. Technol. Mag., vol. 11, no. 2, pp. 43-51, June 2016.

[20] M. Antoniali, A. M. Tonello, M. Lenardon, and A. Qualizza, "Measurements and analysis of PLC channels in a cruise ship," in Proc. IEEE Int. Symp. Power Line Commun. Appl. (ISPLC 2011), Udine, Italy, April 2011, pp. 102-107.

[21] C. H. Jones, "Communications over aircraft power lines," in Proc. IEEE Int. Symp. Power Line Commun. Appl. (ISPLC 2006), Orlando, FL, USA, March 2006, pp. 149-154.

[22] J. Han, C.-S. Choi, W.-K. Park, I. Lee, and S.-H. Kim, "PLC-based photovoltaic system management for smart home energy management system," IEEE Trans. Consum. Electron., vol. 60, no. 2, pp. 184-189, Feb. 2014.

[23] M. Y. Chung, M.-H. Jung, T.-J. Lee, and Y. Lee, "Performance analysis of HomePlug 1.0 MAC with CSMA/CA," IEEE J. Sel. Areas Commun., vol. 24, no. 7, pp. 1411-1420, July 2006.

[24] N. Shlezinger, R. Shaked, and R. Dabora, "On the capacity of MIMO broadband power line communications channels," IEEE Trans. Commun., vol. 66, no. 10, pp. 4795-4810, Oct 2018.
[25] G. Prasad and L. Lampe, "Full-duplex power line communications: Design and applications from multimedia to smart grid," IEEE Commun. Mag., vol. 58, no. 2, pp. 106-112, Feb. 2020.

[26] V. Oksman and S. Galli, "G.hn: The new ITU-T home networking standard," IEEE Commun. Mag., vol. 47, no. 10, pp. 138-145, Oct. 2009.

[27] H. Ma, L. Lampe, and S. Hranilovic, "Hybrid visible light and power line communication for indoor multiuser downlink," IEEE J. Opt. Commun. Netw., vol. 9, no. 8, pp. 635-647, Aug. 2017.

[28] C.-K. Lin, M.-T. Lu, S.-C. Yeh, and H. H. Chen, "Video streaming over in-home power line networks," IEEE Trans. Multimedia, vol. 11, no. 3, pp. 523-534, March 2009.

[29] S. Feng, T. Bai, and L. Hanzo, "Joint power allocation for the multi-user NOMA-downlink in a power-line-fed VLC network," IEEE Transactions on Vehicular Technology, vol. 68, no. 5, pp. 5185-5190, May 2019.

[30] S. Aboagye, A. Ibrahim, T. M. N. Ngatched, A. R. Ndjiongue, and O. A. Dobre, "Design of energy efficient hybrid VLC/RF/PLC communication system for indoor networks," IEEE Wireless Commun. Lett., vol. 9, no. 2, pp. 143-147, Feb. 2020.

[31] L. Zhang, X. Liu, and D. Xu, "A novel security monitoring system of coal mine based on power line communication dynamic routing technology," in Proc. IEEE IAS Annu. Meeting, Vancouver, BC, Canada, 2014, pp. 1-6.

[32] M. Nassar, J. Lin, Y. Mortazavi, A. Dabak, I. H. Kim, and B. L. Evans, "Local utility power line communications in the $3-500 \mathrm{kHz}$ band: Channel impairments, noise, and standards," IEEE Signal Process. Mag., vol. 29, no. 5, pp. 116-127, Sep. 2012.

[33] C. Cano, A. Pittolo, D. Malone, L. Lampe, A. M. Tonello, and A. G. Dabak, "State of the art in power line communications: From the applications to the medium," IEEE J. Sel. Areas Commun., vol. 34 no. 7 , pp. 1935-1952, July 2016.

[34] A. A. Smith, "Power line noise survey," IEEE Trans. Electromagn. Compat., no. 1, pp. 31-32, Jan. 1972.

[35] E. K. Howell, "How switches produce electrical noise," IEEE Trans. Electromagn. Compat., no. 3, pp. 162-170, Aug. 1979.

[36] R. M. Vines, H. J. Trissell, L. J. Gale, and J. B. O'neal, "Noise on residential power distribution circuits," IEEE Trans. Electromagn. Compat., no. 4, pp. 161-168, April 1984.

[37] M. H. L. Chan and R. W. Donaldson, "Amplitude, width, and interarrival distributions for noise impulses on intrabuilding power line communication networks," IEEE Trans. Electromagn. Compat., vol. 31, no. 3, pp. 320-323, Aug. 1989.

[38] M. Arzberger, K. Dostert, T. Waldeck, and M. Zimmermann, "Fundamental properties of the low voltage power distribution grid," in Proc. IEEE Int. Symp. Power Line Commun. Appl. (ISPLC 1997), Essen, German, April 1997.

[39] O. G. Hooijen, "On the channel capacity of the residential power circuit used as a digital communications medium," IEEE Commun. Lett., vol. 2, no. 10, pp. 267-268, Oct. 1998.

[40] M. Zimmermann and K. Dostert, "Analysis and modeling of impulsive noise in broad-band powerline communications," IEEE Trans. Electromagn. Compat., vol. 44, no. 1, pp. 249-258, Feb. 2002.

[41] H. Philipps, "Development of a statistical model for powerline communications channels," in Proc. IEEE Int. Symp. Power Line Commun. Appl. (ISPLC 2000), Limerick, Ireland, April 2000, pp. 5-7.

[42] D. Cooper and T. Jeans, "Narrow-band, low data rate communications on the low-voltage mains in the CENELEC frequencies, Part I: Noise and attenuation," IEEE Power Eng. Rev., vol. 22, no. 4, pp. 75-75, April 2002.

[43] V. Degardin, M. Lienard, A. Zeddam, F. Gauthier, and P. Degauquel, "Classification and characterization of impulsive noise on indoor powerline used for data communications," IEEE Trans. Consum. Electron., vol. 48, no. 4, pp. 913-918, April 2002.

[44] M. Zimmermann and K. Dostert, "A multipath model for the powerline channel," IEEE Trans. Commun., vol. 50, no. 4, pp. 553-559, April 2002.

[45] T. Esmailian, F. R. Kschischang, and P. Glenn Gulak, "In-building power lines as high-speed communication channels: channel characterization and a test channel ensemble," Int. J. Commun. Syst., vol. 16, no. 5, pp. 381-400, May 2003.

[46] D. Benyoucef, "A new statistical model of the noise power density spectrum for powerline communication," Proc. IEEE Int. Symp. Power Line Commun. Appl., pp. 136-141, March 2003.

[47] M. Gotz, M. Rapp, and K. Dostert, "Power line channel characteristics and their effect on communication system design," IEEE Commun. Mag., vol. 42, no. 4, pp. 78-86, April 2004. 
[48] L. Tang, P. So, E. Gunawan, Y. Guan, S. Chen, and T. Lie, "Characterization and modeling of in-building power lines for high-speed data transmission," IEEE Trans. Power Del., vol. 18, no. 1, pp. 69-77, Jan. 2003.

[49] H. Meng, Y. L. Guan, and S. Chen, "Modeling and analysis of noise effects on broadband power-line communications," IEEE Trans. Power Del., vol. 20, no. 2, pp. 630-637, Feb. 2005.

[50] F. Canete, J. Cortes, L. Diez, J. Entrambasaguas, and J. Carmona, "Fundamentals of the cyclic short-time variation of indoor powerline channels," in Proc. IEEE Int.l Symp. Power Line Commun. Appl. (ISPLC 2005), Vancouver, BC, Canada, April 2005, pp. 157-161.

[51] N. Suljanovic, A. Mujcic, M. Zajc, and J. F. Tasic, "Computation of high-frequency and time characteristics of corona noise on HV power line," IEEE Trans. Power Del., vol. 20, no. 1, pp. 71-79, Jan. 2005.

[52] V. Degardin, P. Laly, M. Lienard, and P. Degauque, "Impulsive noise on in-vehicle power lines: Characterization and impact on communication performance," in Proc. IEEE Int. Symp. Power Line Commun. Appl. (ISPLC 2006), Orlando, FL, USA, March 2006, pp. 222-226.

[53] R. Hormis, I. Berenguer, and X. Wang, "A simple baseband transmission scheme for power line channels," IEEE J. Sel. Areas Commun., vol. 24, no. 7, pp. 1351-1363, July 2006.

[54] F. J. C. Corripio, J. A. C. Arrabal, L. D. del Rio, and J. T. E. Munoz, "Analysis of the cyclic short-term variation of indoor power line channels," IEEE J. Sel. Areas Commun., vol. 24, no. 7, pp. 13271338, July 2006.

[55] M. Katayama, T. Yamazato, and H. Okada, "A mathematical model of noise in narrowband power line communication systems," IEEE J. Sel. Areas Commun., vol. 24, no. 7, pp. 1267-1276, July 2006.

[56] Z. Tao, Y. Xiaoxian, Z. Baohui, N. H. Xu, F. Xiaoqun, and L. Changxin, "Statistical analysis and modeling of noise on $10-\mathrm{kV}$ medium-voltage power lines," IEEE Trans. Power Del., vol. 22, no. 3, pp. 1433-1439, March 2007.

[57] S. Tsuzuki, M. Yoshida, Y. Yamada, H. Kawasaki, K. Murai, K. Matsuyama, and M. Suzuki, "Characteristics of power-line channels in cargo ships," in Proc. IEEE Int. Symp. Power Line Commun. Appl. (ISPLC 2007), Pisa, Italy, March 2007, pp. 324-329.

[58] V. Degardin, M. Lienard, P. Degauque, E. Simon, and P. Laly, "Impulsive noise characterization of in-vehicle power line," IEEE Trans. Electromagn. Compat., vol. 50, no. 4, pp. 861-868, April 2008.

[59] J. A. Cortés, L. Diez, F. J. Canete, and J. López, "Analysis of the periodic impulsive noise asynchronous with the mains in indoor PLC channels," in Proc. IEEE Int. Symp. Power Line Commun. Appl. (ISPLC 2009), Dresden, Germany, March 2009, pp. 26-30

[60] J. A. Cortes, L. Diez, F. J. Canete, and J. J. Sanchez-Martinez, "Analysis of the indoor broadband power-line noise scenario," IEEE Trans. Electromagn. Compat., vol. 52, no. 4, pp. 849-858, Nov. 2010.

[61] N. Andreadou and F.-N. Pavlidou, "Modeling the noise on the OFDM power-line communications system," IEEE Trans. Power Del., vol. 25, no. 1, pp. 150-157, Jan. 2010.

[62] D. Rende, A. Nayagam, K. Afkhamie, L. Yonge, R. Riva, D. Veronesi, F. Osnato, and P. Bisaglia, "Noise correlation and its effect on capacity of inhome MIMO power line channels," in Proc. IEEE Int. Symp. Power Line Commun. Appl. (ISPLC 2011), Udine, Italy, April 2011, pp. 6065.

[63] D. Guezgouz, D. E. Chariag, Y. Raingeaud, and J.-C. Le Bunetel, "Modeling of electromagnetic interference and PLC transmission for loads shedding in a microgrid," IEEE Trans. Power Electron., vol. 26, no. 3, pp. 747-754, March 2011.

[64] L. Di Bert, P. Caldera, D. Schwingshackl, and A. M. Tonello, "On noise modeling for power line communications," in Proc. IEEE Int.l Symp. Power Line Commun. Appl. (ISPLC 2011), Udine, Italy, April 2011, pp. 283-288.

[65] M. Nassar, K. Gulati, Y. Mortazavi, and B. L. Evans, "Statistical modeling of asynchronous impulsive noise in powerline communication networks," in Proc. IEEE Global Telecomm. Conf. (GLOBECOM 2011), Kathmandu, Nepal, Dec. 2011, pp. 1-6.

[66] R. Hashmat, P. Pagani, T. Chonavel, and A. Zeddam, "Analysis and modeling of background noise for inhome MIMO PLC channels," in Proc. IEEE Int. Symp. Power Line Commun. Appl. (ISPLC 2012), Beijing, China, March 2012, pp. 316-321.

[67] M. Nassar, A. Dabak, I. H. Kim, T. Pande, and B. L. Evans, "Cyclostationary noise modeling in narrowband powerline communication for smart grid applications," in Proc. IEEE Int. Conf. Acoust., Speech, Sig. Process. (ICASSP 2012), Kyoto, Japan, March 2012, pp. 3089-3092.

[68] S. Barmada, M. Raugi, R. Rizzo, and M. Tucci, "Channel evaluation for power line communication in plug-in electric vehicles," IET Electr. Syst. Transport., vol. 2, no. 4, pp. 195-201, April 2012.
[69] R. Hashmat, P. Pagani, T. Chonavel, and A. Zeddam, "A time-domain model of background noise for in-home MIMO PLC networks," IEEE Trans. Power Del., vol. 27, no. 4, pp. 2082-2089, April 2012.

[70] F. Gianaroli, F. Pancaldi, E. Sironi, M. Vigilante, G. M. Vitetta, and A. Barbieri, "Statistical modeling of periodic impulsive noise in indoor power-line channels," IEEE Trans. Power Del., vol. 27, no. 3, pp. 12761283, July 2012

[71] M. Antoniali, M. De Piante, and A. M. Tonello, "PLC noise and channel characterization in a compact electrical car," in Proc. IEEE Int. Symp. Power Line Commun. Appl. (ISPLC 2013), Johannesburg, South Africa, March 2013, pp. 29-34.

[72] A. N. Milioudis, K. N. Syranidis, G. T. Andreou, and D. P. Labridis, "Modeling of medium-voltage power-line communication systems noise levels," IEEE Trans. Power Del., vol. 28, no. 4, pp. 2004-2013, April 2013.

[73] F. Sacuto, F. Labeau, and B. L. Agba, "Wide band time-correlated model for wireless communications under impulsive noise within power substation," IEEE Trans. Wireless Commun., vol. 13, no. 3, pp. 1449-1461, March 2014.

[74] L. Guerrieri, G. Masera, I. S. Stievano, P. Bisaglia, W. R. G. Valverde, and M. Concolato, "Automotive power-line communication channels: Mathematical characterization and hardware emulator," IEEE Trans. Ind. Electron., vol. 63, no. 5, pp. 3081-3090, May 2016.

[75] M. Antoniali, F. Versolatto, and A. M. Tonello, "An experimental characterization of the PLC noise at the source," IEEE Trans. Power Del., vol. 31, no. 3, pp. 1068-1075, June 2016.

[76] F. J. Cañete, L. Diez, J. A. Cortes, J. J. Sanchez-Martinez, and L. M. Torres, "Time-varying channel emulator for indoor power line communications," in Proc. IEEE Global Telecommun. Conf. (GLOBECOM 2008), New Orleans, LO, USA, Dec. 2008, pp. 1-5.

[77] N. Weling, "Flexible FPGA-based powerline channel emulator for testing MIMO-PLC, neighborhood networks, hidden node or VDSL coexistence scenarios," in Proc. IEEE Int. Symp. Power Line Commun. Appl. (ISPLC 2011), Udine, Italy, April 2011, pp. 12-17.

[78] N. Weling, A. Engelen, and S. Thiel, "Broadband MIMO powerline channel emulator verification and testing results," in Proc. IEEE Int. Symp. Power Line Commun. Appl. (ISPLC 2015), Austin, TX, USA., April 2015, pp. 59-64.

[79] B. Han, V. Stoica, C. Kaiser, N. Otterbach, and K. Dostert, "Noise characterization and emulation for low-voltage power line channels across narrowband and broadband," Digital Signal Process., vol. 69 , pp. 259-274, July 2017.

[80] T. Shongwe, A. H. Vinck, and H. C. Ferreira, "On impulse noise and its models," in Proc. IEEE Int. Symp. Power Line Commun. Appl. (ISPLC 2014), Glasgow, UK, April 2014, pp. 12-17.

[81] P. D. Mariyam, F. H. Juwono, P. D. Pamungkasari, and D. Gunawan, "How to deal with impulsive noise in OFDM-based PLC: A survey," in Proc. IEEE Int. Conf. Electr. Eng. and Informat. (ICELTICs 2017), Banda Aceh, Indonesia, Oct. 2017, pp. 163-168.

[82] S. Laksir, A. Chaoub, and A. Tamtaoui, "Impulsive noise reduction techniques in power line communication: A survey and recent trends," in Proc. Int. Conf. Sel. Topics Mob. Wireless Netw. (MoWNeT), Tangier, Morocco, 2018, pp. 13-20.

[83] L. T. Berger, A. Schwager, P. Pagani, and D. M. Schneider, "MIMO power line communications," IEEE Commun. Surveys Tuts., vol. 17, no. 1, pp. 106-124, Jan. 2015.

[84] T. Bai, H. Zhang, J. Zhang, C. Xu, A. F. Al Rawi, and L. Hanzo, "Impulsive noise mitigation in digital subscriber lines: The state-ofthe-art and research opportunities," IEEE Communications Magazine, vol. 57, no. 5, pp. 145-151, May 2019.

[85] S. Wang, Z. He, K. Niu, P. Chen, and Y. Rong, "New results on joint channel and impulsive noise estimation and tracking in underwater acoustic OFDM systems," IEEE Transactions on Wireless Communications, vol. 19, no. 4, pp. 2601-2612, April 2020.

[86] K. L. Blackard, T. S. Rappaport, and C. W. Bostian, "Measurements and models of radio frequency impulsive noise for indoor wireless communications," IEEE J. Sel. Areas Commun., vol. 11, no. 7, pp. 991-1001, Sept. 1993.

[87] M. H. Chan and R. W. Donaldson, "Attenuation of communication signals on residential and commercial intrabuilding power-distribution circuits," IEEE Trans. Electromagn. Compat., vol. 28, no. 4, pp. 220230, April 1986.

[88] M. Tanaka, "Transmission characteristics of a power line used for data communications at high frequencies," IEEE Trans. Consum. Electron., vol. 35, no. 1, pp. 37-42, Jan. 1989. 
[89] H. Philipps, "Modelling of powerline communication channels," in Proc. IEEE Int. Symp. Power-line Commun. Appl. (ISPLC 1999), Lancaster, UK, April 1999, pp. 14-21.

[90] F. J. Canete, L. Diez, J. A. Cortes, and J. T. Entrambasaguas, "Broadband modelling of indoor power-line channels," IEEE Trans. Consum. Electron., vol. 48, no. 1, pp. 175-183, Jan. 2002.

[91] A. M. Tonello and F. Versolatto, "Bottom-up statistical PLC channel modeling Part I: Random topology model and efficient transfer function computation," IEEE Trans. Power Del., vol. 26, no. 2, pp. 891-898, April 2011

[92] F. J. Canete, J. A. Cortes, L. Diez, and J. T. Entrambasaguas, "A channel model proposal for indoor power line communications," IEEE Commun. Mag., vol. 49, no. 12, pp. 166-174, Dec. 2011.

[93] W. Liu, M. Sigle, and K. Dostert, "Channel characterization and system verification for narrowband power line communication in smart grid applications," IEEE Commun. Mag., vol. 49, no. 12, pp. 28-35, Dec. 2011.

[94] F. Passerini, D. Righini, and A. M. Tonello, "A bottom-up PLC channel model that includes radiation effects," in Proc. IEEE Int. Symp. Powerline Commun. Appl. (ISPLC 2018), Manchester, UK, April 2018, pp. $1-6$.

[95] D. Righini, F. Passerini, and A. M. Tonello, "Modeling transmission and radiation effects when exploiting power line networks for communication," IEEE Trans. Electromagn. Compat., vol. 60, no. 1, pp. 59-67, Feb. 2018.

[96] S. Souissi, O. B. Rhouma, and C. Rebai, "Bottom-up approach for narrowband powerline channel modeling," in Proc. IEEE Global Telecommun. Conf. (GLOBECOM 2013), Atlanta, GA, USA, Dec 2013, pp. 2987-2992.

[97] F. Versolatto and A. M. Tonello, "An MTL theory approach for the simulation of MIMO power-line communication channels," IEEE Trans. Power Del., vol. 26, no. 3, pp. 1710-1717, March 2011.

[98] V. Oksman and J. Zhang, "G. HNEM: the new ITU-T standard on narrowband PLC technology," IEEE Commun. Mag., vol. 49, no. 12, pp. 36-44, Dec. 2011.

[99] S. Morosi, D. Marabissi, E. Del Re, R. Fantacci, and N. Del Santo, "A rate adaptive bit-loading algorithm for in-building power-line communications based on DMT-modulated systems," IEEE Trans. Power Del., vol. 21, no. 4, pp. 1892-1897, April 2006.

[100] D. Raphaeli, "Noncoherent coded modulation," IEEE Trans. Commun., vol. 44, no. 2, pp. 172-183, Feb. 1996

[101] E. N. Gilbert, "Capacity of a burst-noise channel," Bell Syst. Tech. J., vol. 39, no. 5, pp. 1253-1265, May 1960

[102] E. O. Elliott, "Estimates of error rates for codes on burst-noise channels," Bell Syst. Tech. J., vol. 42, no. 5, pp. 1977-1997, May 1963.

[103] D. Middleton, "Statistical-physical models of electromagnetic interference," IEEE Trans. Electromagn. Compat., no. 3, pp. 106-127, March 1977.

[104] M. Ghosh, "Analysis of the effect of impulse noise on multicarrier and single carrier QAM systems," IEEE Trans. Commun., vol. 44, no. 2 , pp. 145-147, Feb. 1996

[105] D. Middleton, "Non-Gaussian noise models in signal processing for telecommunications: new methods an results for class A and class B noise models," IEEE Trans. Inf. Theory, vol. 45, no. 4, pp. 1129-1149, April 1999.

[106] R. Pighi, M. Franceschini, G. Ferrari, and R. Raheli, "Fundamental performance limits for PLC systems impaired by impulse noise," in Proc. IEEE Int. Symp. Power Line Commun. Appl. (ISPLC 2006), Orlando, FL, USA, March 2006, pp. 277-282.

[107] D. Fertonani and G. Colavolpe, "On reliable communications over channels impaired by bursty impulse noise," IEEE Trans. Commun., vol. 57, no. 7, July 2009.

[108] I. H. Kim, B. Varadarajan, and A. Dabak, "Performance analysis and enhancements of narrowband OFDM powerline communication systems," in Proc. IEEE Int. Conf. Smart Grid Commun. (SmartGridComm 2010), Gaithersburg, MD, USA, Oct. 2010, pp. 362-367.

[109] J. Mitra and L. Lampe, "Convolutionally coded transmission over Markov-Gaussian channels: Analysis and decoding metrics," IEEE Trans. Commun., vol. 58, no. 7, pp. 1939-1949, July 2010.

[110] J. Wang, E. E. Kuruoglu, and T. Zhou, "Alpha-stable channel capacity," IEEE Commun. Lett., vol. 15, no. 10, pp. 1107-1109, Oct. 2011.

[111] G. Ndo, F. Labeau, and M. Kassouf, "A Markov-Middleton model for bursty impulsive noise: Modeling and receiver design," IEEE Trans. Power Del., vol. 28, no. 4, pp. 2317-2325, April 2013

[112] M. Mirahmadi, A. Al-Dweik, and A. Shami, "BER reduction of OFDM based broadband communication systems over multipath channels with impulsive noise," IEEE Trans. Commun., vol. 61, no. 11, pp. 46024615, Nov. 2013.

[113] G. Laguna-Sanchez and M. Lopez-Guerrero, "An experimental study of the effect of human activity on the alpha-stable characteristics of the power-line noise," in Proc. IEEE Int. Symp. Power Line Commun. Appl. (ISPLC 2014), Glasgow, UK, April 2014, pp. 6-11.

[114] _ "On the use of alpha-stable distributions in noise modeling for PLC," IEEE Trans. Power Del., vol. 30, no. 4, pp. 1863-1870, Aug. 2015

[115] S. P. Herath, N. H. Tran, and T. Le-Ngoc, "Optimal signaling scheme and capacity limit of PLC under Bernoulli-Gaussian impulsive noise," IEEE Trans. Power Del., vol. 30, no. 1, pp. 97-105, Jan. 2015.

[116] A. Mathur, M. R. Bhatnagar, and B. K. Panigrahi, "Performance evaluation of PLC under the combined effect of background and impulsive noises," IEEE Commun. Lett., vol. 19, no. 7, pp. 1117-1120, July 2015.

[117] H. Zhang, L.-L. Yang, and L. Hanzo, "Performance analysis of orthogonal frequency division multiplexing systems in dispersive indoor power line channels inflicting asynchronous impulsive noise," IET Commun., vol. 10, no. 5, pp. 453-461, May 2016.

[118] J. A. Cortés, A. Sanz, P. Estopinán, and J. I. García, "On the suitability of the Middleton class A noise model for narrowband PLC", in Proc. IEEE Int. Symp. Power Line Commun. Appl. (ISPLC 2016), Bottrop, Germany, March 2016, pp. 58-63.

[119] S. Liu, F. Yang, and J. Song, "An optimal interleaving scheme with maximum time-frequency diversity for PLC systems," IEEE Trans. Power Del., vol. 31, no. 3, pp. 1007-1014, June 2016.

[120] T. Bai, H. Zhang, R. Zhang, L.-L. Yang, A. F. Al Rawi, J. Zhang, and L. Hanzo, "Discrete multi-tone digital subscriber loop performance in the face of impulsive noise," IEEE Access, vol. 5, pp. 10 478-10495, 2017.

[121] L. Bai, M. Tucci, S. Barmada, M. Raugi, and T. Zheng, "Impulsive noise characterization in narrowband power line communication," Energies, vol. 11, no. 4, p. 863, April 2018.

[122] A. Papoulis and S. U. Pillai, Probability, random variables, and stochastic processes. Tata McGraw-Hill Education, 2002.

[123] L. T. Berger, A. Schwager, P. Pagani, and D. Schneider, MIMO power line communications: narrow and broadband standards, EMC, and advanced processing. CRC Press, 2014

[124] A. Dabak, B. Varadrajan, I. H. Kim, M. Nassar, and G. Gregg, "Appendix for noise channel modeling for IEEE 1901.2," IEEE P1901.2, 2011

[125] L. R. Rabiner and B. Gold, "Theory and application of digital signal processing," Englewood Cliffs, NJ, Prentice-Hall, Inc., 1975. 777 p., 1975.

[126] S. M. Kay, "Fundamentals of statistical signal processing, volume I: Estimation theory," PTR Prentice-Hall, Englewood Cliffs, 1993.

[127] K. W. Hipel and A. I. McLeod, Time series modelling of water resources and environmental systems. Elsevier, 1994, vol. 45.

[128] P. J. Brockwell and R. A. Davis, Time series: theory and methods. Springer Science \& Business Media, 2013.

[129] A. Schiffer, "Statistical channel and noise modeling of vehicular DClines for data communication," in Proc. IEEE Veh. Technol. Conf (VTC2000-Spring), vol. 1, Tokyo, Japan, May 2000, pp. 158-162 vol.1.

[130] J. O. Onunga and R. W. Donaldson, "A simple packet retransmission strategy for throughput and delay enhancement on power line communication channels," IEEE Trans. Power Del., vol. 8, no. 3, pp. 818-826, March 1993.

[131] R. Haring and A. H. Vinck, "Performance bounds for optimum and suboptimum reception under Class-A impulsive noise," IEEE Trans. Commun., vol. 50, no. 7, pp. 1130-1136, July 2002.

[132] E. Biglieri, "Coding and modulation for a horrible channel," IEEE Commun. Mag., vol. 41, no. 5, pp. 92-98, May 2003.

[133] D. Toumpakaris, J. M. Cioffi, and D. Gardan, "Reduced-delay protection of DSL systems against nonstationary disturbances," IEEE Trans. Commun., vol. 52, no. 11, pp. 1927-1938, Nov. 2004.

[134] D. Umehara, H. Yamaguchi, and Y. Morihiro, "Turbo decoding in impulsive noise environment," in Proc. IEEE Global Telecommun. Conf. (GLOBECOM 2004), vol. 1, Dallas, TX, USA, Dec. 2004, pp. 194-198.

[135] H. Nakagawa, D. Umehara, S. Denno, and Y. Morihiro, "A decoding for low density parity check codes over impulsive noise channels," in Proc. IEEE Int. Symp. Power Line Commun. Appl. (ISPLC 2005), Vancouver, BC, Canada, April 2005, pp. 85-89.

[136] M. Ardakani, F. R. Kschischang, and W. Yu, "Low-density parity-check coding for impulse noise correction on power-line channels," in Proc. 
IEEE Int. Symp. Power Line Commun. Appl. (ISPLC 2005), Vancouver, BC, Canada, April 2005, pp. 90-94.

[137] S. Katar, L. Yonge, R. Newman, and H. Latchman, "Efficient framing and ARQ for high-speed PLC systems," in Proc. IEEE Int. Symp. Power Line Commun. Appl. (ISPLC 2005), Vancouver, BC, Canada, April 2005, pp. 27-31.

[138] L. Guerrieri, P. Bisaglia, G. Dell'Amico, and E. Guerrini, "Performance of the turbo coded HomePlug AV system over power-line channels," in Proc. IEEE Int. Symp. Power Line Commun. Appl. (ISPLC 2007), Pisa, Italy, March 2007, pp. 138-143

[139] A. Papaioannou, G. D. Papadopoulos, and F. Pavlidou, "Hybrid ARQ combined with distributed packet space-time block coding for multicast power-line communications," IEEE Trans. Power Del., vol. 23, no. 4, pp. 1911-1917, April 2008.

[140] S. V. Zhidkov, "Analysis and comparison of several simple impulsive noise mitigation schemes for OFDM receivers," IEEE Trans. Commun., vol. 56, no. 1, Jan. 2008.

[141] T. Li, W. H. Mow, and M. Siu, "Joint erasure marking and list Viterbi algorithm for decoding in unknown non-Gaussian noise," IEEE Trans. Wireless Commun., vol. 7, no. 3, pp. 787-792, July 2008.

[142] G. Caire, and T. Y. Al-Naffouri, and A. K. Narayanan,, "Impulse noise cancellation in OFDM: an application of compressed sensing," in Proc. IEEE Int. Symp. Inf. Theory (ISIT 2008), Toronto, ON, Canada, July 2008, pp. 1293-1297.

[143] J. Mitra and L. Lampe, "On joint estimation and decoding for channels with noise memory," IEEE Commun. Lett., vol. 13, no. 10, 2009.

[144] A. Al-Dweik, A. Hazmi, B. Sharif, and C. Tsimenidis, "Efficient interleaving technique for OFDM system over impulsive noise channels," in Proc. IEEE Int. Symp. Pers., Indoor Mobile Radio Commun. (PIMRC 2010), Instanbul, Turkey, Sept. 2010, pp. 167-171.

[145] M. Nassar, K. Gulati, M. R. DeYoung, B. L. Evans, and K. R. Tinsley, "Mitigating near-field interference in laptop embedded wireless transceivers," J. Signal Process. Syst., vol. 63, no. 1, pp. 1-12, Jan. 2011.

[146] D.-F. Tseng, Y. S. Han, W. H. Mow, L.-C. Chang, and A. H. Vinck, "Robust clipping for OFDM transmissions over memoryless impulsive noise channels," IEEE Commun. Lett., vol. 16, no. 7, pp. 1110-1113, July 2012.

[147] J. Lin and B. L. Evans, "Cyclostationary noise mitigation in narrowband powerline communications," in Proc. APSIPA Annu. Summit Conf. (APSIPA ASC 2012), Hollywood, CA, USA, Dec. 2012, pp. 1-4.

[148] V. N. Papilaya and A. H. Vinck, "Investigation on a new combined impulsive noise mitigation scheme for OFDM transmission," in Proc. IEEE Int. Symp. Power Line Commun. Appl. (ISPLC 2013), Johannesburg, South Africa, March 2013, pp. 86-91.

[149] N. Shlezinger and R. Dabora, "Frequency-shift filtering for OFDM signal recovery in narrowband power line communications," IEEE Trans. Commun., vol. 62, no. 4, pp. 1283-1295, April 2014.

[150] K. Rabie, E. Alsusa, A. Familua, and L. Cheng, "Constant envelope OFDM transmission over impulsive noise power-line communication channels," in Proc. IEEE Int. Symp. Power Line Commun. Appl. (ISPLC 2015), Austin, TX, USA, April 2015, pp. 13-18.

[151] M. Elgenedy, M. Sayed, M. Mokhtar, M. Abdallah, and N. Al-Dhahir, "Interference mitigation techniques for narrowband powerline smart grid communications," in Proc. IEEE Int. Conf. Smart Grid Commun. (SmartGridComm 2015), Miami, FL, USA, Nov. 2015, pp. 368-373.

[152] H. Zhang, L. L. Yang, and L. Hanzo, "Compressed impairment sensing-assisted and interleaved-double-FFT-aided modulation improves broadband power line communications subjected to asynchronous impulsive noise," IEEE Access, vol. 4, pp. 81-96, 2016.

[153] A. Hadi, K. M. Rabie, and E. Alsusa, "Polar codes based OFDM-PLC systems in the presence of Middleton class-A noise," in Proc. IEEE Int. Symp. Commun. Syst., Netw. Digital Signal Process. (CSNDSP 2016), Prague, Czech Republic, July 2016, pp. 1-6.

[154] T. Bai, C. Xu, R. Zhang, A. F. Al Rawi, and L. Hanzo, "Joint impulsive noise estimation and data detection conceived for LDPC-coded DMTbased DSL systems," IEEE Access, vol. 5, pp. 23 133-23 145, May 2017.

[155] J. Yin, X. Zhu, Y. Huang, and Y. Jiang, "Null subcarriers assisted impulsive noise mitigation for in-vehicle power line communication in the presence of narrowband interference," IEEE Trans. Veh. Technol., vol. 68, no. 1, pp. 498-508, Jan. 2019.

[156] L. Bai, M. Tucci, and M. Raugi, "Impulsive noise mitigation with interleaving based on MUSIC in power line communication," IEEE Transactions on Smart Grid, vol. 10, no. 4, pp. 3575-3584, July 2019.
[157] N. Andreadou and A. M. Tonello, "On the mitigation of impulsive noise in power-line communications with LT codes," IEEE Trans. Power Del., vol. 28, no. 3, pp. 1483-1490, March 2013.

[158] G. Caire, G. Taricco, and E. Biglieri, "Bit-interleaved coded modulation," IEEE Trans. Inf. Theory, vol. 44, no. 3, pp. 927-946, March 1998.

[159] A. Nasri and R. Schober, "Performance of BICM-SC and BICMOFDM systems with diversity reception in non-Gaussian noise and interference," IEEE Trans. Commun., vol. 57, no. 11, Nov. 2009.

[160] H. H. Nguyen and T. Q. Bui, "Bit-interleaved coded modulation with iterative decoding in impulsive noise," IEEE Trans. Power Del., vol. 22, no. 1, pp. 151-160, Jan. 2007.

[161] S. Nayyef, C. Tsimenidis, A. Al-Dweik, B. Sharif, and A. Hazmi, "Time-and frequency-domain impulsive noise spreader for OFDM systems," in Proc. IEEE Int. Conf. Trust, Security Privacy Comput. Commun. (TrustCom 2012), Liverpool, UK, June 2012, pp. 1856-1861.

[162] J. Lin, M. Nassar, and B. L. Evans, "Impulsive noise mitigation in powerline communications using sparse bayesian learning," IEEE $J$. Sel. Areas Commun., vol. 31, no. 7, pp. 1172-1183, July 2013.

[163] G. Ndo, P. Siohan, M.-H. Hamon, and J. Horard, "Optimization of turbo decoding performance in the presence of impulsive noise using soft limitation at the receiver side," in Proc. IEEE Global Telecommun. Conf. (GLOBECOM 2008), New Orleans, LO, USA, Dec. 2008, pp. $1-5$.

[164] G. Ndo, P. Siohan, and M.-H. Hamon, "Adaptive noise mitigation in impulsive environment: Application to power-line communications," IEEE Trans. Power Del., vol. 25, no. 2, pp. 647-656, Feb. 2010.

[165] M. Korki, N. Hosseinzadeh, H. L. Vu, T. Moazzeni, and C. H. Foh, "Impulsive noise reduction of a narrowband power line communication using optimal nonlinearity technique," in Proc. IEEE Aust. Telecommun. Netw. Appl. Conf. (ATNAC 2011), Melbourne, VIC, Australia, Nov. 2011, pp. 1-4.

[166] E. Alsusa and K. M. Rabie, "Dynamic peak-based threshold estimation method for mitigating impulsive noise in power-line communication systems," IEEE Trans. Power Del., vol. 28, no. 4, pp. 2201-2208, April 2013.

[167] F. H. Juwono, Q. Guo, D. Huang, and K. P. Wong, "Joint peak amplitude and impulsive noise clippings in OFDM-based power line communications," in Proc. IEEE Asia-Pacific Conf. Commun. (APCC 2013), Denpasar, Indonesia, Aug. 2013, pp. 567-571.

[168] — - "Deep clipping for impulsive noise mitigation in OFDM-based power-line communications," IEEE Trans. Power Del., vol. 29, no. 3, pp. 1335-1343, March 2014.

[169] K. M. Rabie and E. Alsusa, "Preprocessing-based impulsive noise reduction for power-line communications," IEEE Trans. Power Del., vol. 29, no. 4, pp. 1648-1658, April 2014

[170] Y. G. Yoo and J. H. Cho, "Asymptotic analysis of CP-SC-FDE and UWSC-FDE in additive cyclostationary noise," in Proc. IEEE Int. Conf. Commun. (ICC 2008), Beijing, China, May 2008, pp. 1410-1414.

[171] R. García, L. Díez, J. A. Cortés, and F. J. Cañete, "Mitigation of cyclic short-time noise in indoor power-line channels," in Proc. IEEE Int. Symp. Power Line Commun. Appl. (ISPLC 2007), Pisa, Italy, March 2007, pp. 396-400.

[172] A. Llano, A. Sendin, A. Arzuaga, and S. Santos, "Quasi-synchronous noise interference cancellation techniques applied in low voltage PLC," in Proc. IEEE Int. Symp. Power Line Commun. Appl. (ISPLC 2011), Udine, Italy, April 2011, pp. 108-112.

[173] S. Miyamoto, M. Katayama, and N. Morinaga, "Performance analysis of QAM systems under class A impulsive noise environment," IEEE Trans. Electromagn. Compat., vol. 37, no. 2, pp. 260-267, Feb. 1995.

[174] T. Fukami, D. Umehara, M. Kawai, and Y. Morihiro, "Noncoherent PSK optimum receiver over impulsive noise channels," in Proc. IEEE Int. Symp. Power Line Commun. Appl. (ISPLC 2002), Athens, Greece, March 2002, pp. 235-238.

[175] M. Nassar and B. L. Evans, "Low complexity EM-based decoding for OFDM systems with impulsive noise," in Proc. IEEE Asilomar Conf. Signals, Syst. Comput. (ASILOMAR 2011), Pacific Grove, CA, USA, Nov. 2011, pp. 1943-1947.

[176] J. Haring and A. H. Vinck, "Iterative decoding of codes over complex numbers for impulsive noise channels," IEEE Trans. Inf. Theory, vol. 49, no. 5, pp. 1251-1260, May 2003.

[177] F. Abdelkefi, P. Duhamel, and F. Alberge, "Impulsive noise cancellation in multicarrier transmission," IEEE Trans. Commun., vol. 53, no. 1, pp. 94-106, Jan. 2005.

[178] H.-M. Oh, Y.-J. Park, S. Choi, J.-J. Lee, and K.-C. Whang, "Mitigation of performance degradation by impulsive noise in LDPC coded OFDM 
system," in Proc. IEEE Int. Symp. Power Line Commun. Appl. (ISPLC 2006), Orlando, FL, USA, March 2006, pp. 331-336.

[179] K. Leung and L. Welch, "Erasure decoding in burst-error channels," IEEE Trans. Inf. Theory, vol. 27, no. 2, pp. 160-167, Feb. 1981.

[180] M. Luby, "LT codes," in Proc. IEEE Symp. Foundations Computer Sci. (FOCS 2002), Vancouver, BC, Canada, Nov. 2002, pp. 271-280.

[181] T. Li, W. H. Mow, and M. H. Siu, "A joint approach to erasure marking and Viterbi decoding for impulsive noise channels," in Proc. IEEE Workshop Signal Process. Advances Wireless Commun. (SPAWC 2003), Rome, Italy, July 2003, pp. 180-184.

[182] T. Li, W. H. Mow, V. K. Lau, M. Siu, R. S. Cheng, and R. D. Murch, "Robust joint interference detection and decoding for OFDM-based cognitive radio systems with unknown interference," IEEE J. Sel. Areas Commun., vol. 25, no. 3, March 2007.

[183] L. Lampe, "Bursty impulse noise detection by compressed sensing," in Proc. IEEE Int. Symp. Power Line Commun. Appl. (ISPLC 2011), Udine, Italy, April 2011, pp. 29-34.

[184] G. Ren, S. Qiao, and Y. Hei, "Asynchronous impulsive noise mitigation in OFDM using adaptive threshold compressive sensing," in Proc. IEEE Wireless Microw. Technol. Conf. (WAMICON 2014), Tampa, FL, USA, June 2014, pp. 1-5.

[185] S. Liu, F. Yang, W. Ding, and J. Song, "Double kill: Compressivesensing-based narrow-band interference and impulsive noise mitigation for vehicular communications." IEEE Trans. Veh. Technol., vol. 65, no. 7, pp. 5099-5109, June 2016.

[186] M. Korki, J. Zhang, C. Zhang, and H. Zayyani, "Block-sparse impulsive noise reduction in OFDM systems-A novel iterative Bayesian approach," IEEE Trans. Commun., vol. 64, no. 1, pp. 271-284, Jan. 2016.

[187] S. Liu, F. Yang, W. Ding, J. Song, and A. M. Tonello, "Structured compressed sensing based narrowband interference elimination for inhome power line communications," IEEE Trans. Consum. Electron., vol. 63, no. 1, pp. 10-18, Feb. 2017.

[188] I. S. Reed and G. Solomon, "Polynomial codes over certain finite fields," J. Society Ind. Appl. Math., vol. 8, no. 2, pp. 300-304, Feb. 1960.

[189] C. Berrou, A. Glavieux, and P. Thitimajshima, "Near shannon limit error-correcting coding and decoding: Turbo-codes (1)," in Proc. IEEE Int. Conf. Commun. (ICC 1993), vol. 2, Geneva, Switzerland, May 1993, pp. 1064-1070.

[190] R. Gallager, "Low-density parity-check codes," IRE Trans. Inf. Theory, vol. 8, no. 1, pp. 21-28, Jan. 1962.

[191] E. Arikan, "Channel polarization: A method for constructing capacityachieving codes for symmetric binary-input memoryless channels," IEEE Trans. Inf. Theory, vol. 55, no. 7, pp. 3051-3073, July 2009.

[192] S. Lin, D. J. Costello, and M. J. Miller, "Automatic-repeat-request error-control schemes," IEEE Commun. Mag., vol. 22, no. 12, pp. 5-17, Dec. 1984.

[193] S. Lin, D. J. Costello Jr, and M. J. Miller, "Automatic-repeat-request error control schemes," IEEE Commun. Mag., 1983.

[194] D. Chase, "Code combining-a maximum-likelihood decoding approach for combining an arbitrary number of noisy packets," IEEE Trans. Commun., vol. 33, no. 5, pp. 385-393, May 1985.

[195] S. Sesia, G. Caire, and G. Vivier, "Incremental redundancy hybrid ARQ schemes based on low-density parity-check codes," IEEE Trans. Commun., vol. 52, no. 8, pp. 1311-1321, Aug. 2004.

[196] P. Wu and N. Jindal, "Performance of hybrid-ARQ in block-fading channels: A fixed outage probability analysis," IEEE Trans. Commun., vol. 58, no. 4, pp. 1129-1141, April 2010.

[197] C.-H. Yih, "Iterative interference cancellation for OFDM signals with blanking nonlinearity in impulsive noise channels," IEEE Signal Process. Lett., vol. 19, no. 3, pp. 147-150, March 2012.

[198] A. Mengi and A. H. Vinck, "Successive impulsive noise suppression in OFDM," in Proc. Int. Symp. Power Line Commun. Appl. (ISPLC 2010). Rio de Janeiro, Brazil: IEEE, March 2010, pp. 33-37.

[199] J. A. Davis and J. Jedwab, "Peak-to-mean power control in OFDM, Golay complementary sequences, and Reed-Muller codes," IEEE Trans. Inf. Theory, vol. 45, no. 7, pp. 2397-2417, July 1999.

[200] J. Tellado, "Peak-to-average power reduction for multicarrier modulation," Ph.D. dissertation, Stanford University, Stanford, CA, USA, 1999.

[201] J. Armstrong, "Peak-to-average power reduction for OFDM by repeated clipping and frequency domain filtering," Electron. Lett., vol. 38, no. 5, pp. 246-247, May 2002.

[202] L. Wang and C. Tellambura, "A simplified clipping and filtering technique for PAR reduction in OFDM systems," IEEE Signal Process. Lett., vol. 12, no. 6, pp. 453-456, June 2005.
[203] Y.-C. Wang and Z.-Q. Luo, "Optimized iterative clipping and filtering for papr reduction of ofdm signals," IEEE Trans. Commun., vol. 59, no. 1, pp. 33-37, Jan. 2010.

[204] X. Zhu, W. Pan, H. Li, and Y. Tang, "Simplified approach to optimized iterative clipping and filtering for PAPR reduction of OFDM signals," IEEE Trans. Commun., vol. 61, no. 5, pp. 1891-1901, May 2013.

[205] K. M. Rabie and E. Alsusa, "Single-carrier FDMA with blanking/clipping for mitigating impulsive noise over PLC channels," in Proc. IEEE Int. Symp. Power Line Commun. Appl. (ISPLC 2014), Glasgow, UK, April 2014, pp. 340-345.

[206] E. Guerrini, L. Guerrni, and D. Veronesi, "HomePlug AV system and DLC bit-loading algorithm over OPERA power-line channels with impulsive noise," in Proc. IEEE Int. Symp. Power Line Commun. Appl. (ISPLC 2008), Jeju City, South Korea, April 2008, pp. 164-169.

[207] L. Hanzo, J. P. Woodard, and P. Robertson, "Turbo decoding and detection for wireless applications," Proc. IEEE, vol. 95, no. 6, pp. 1178-1200, June 2007.

[208] N. Seshadri and C.-E. Sundberg, "List Viterbi decoding algorithms with applications," IEEE Trans. Commun., vol. 42, no. 3, pp. 313-323, Aug. 1994.

[209] "IEEE standard for broadband over power line networks: medium access control and physical layer specifications," IEEE Standard 1901, vol. 2010, pp. 1-1586, 2010.

[210] HomePlug Powerline Alliance, HomePlug AV2 White Paper, 2013, available at http://www.homeplug.org/.

[211] J. Rinne, A. Hazmi, and M. Renfors, "Impulse burst position detection and channel estimation schemes for OFDM systems," IEEE Trans. Consum. Electron., vol. 49, no. 3, pp. 539-545, March 2003.

[212] M. Raugi and M. Tucci, "Power-line communications channel estimation and tracking by a competitive neural network," IEEE Trans Consum. Electron., vol. 52, no. 4, April 2006.

[213] A. Mehboob, L. Zhang, J. Khangosstar, and K. Suwunnapuk, "Joint channel and impulsive noise estimation for OFDM based power line communication systems using compressed sensing," in Proc. IEEE Int. Symp. Power Line Commun. Appl. (ISPLC 2013), Johannesburg, South Africa, March 2013, pp. 203-208.

[214] M. Nassar, P. Schniter, and B. L. Evans, "A factor graph approach to joint OFDM channel estimation and decoding in impulsive noise environments," IEEE Trans. Signal Process., vol. 62, no. 6, pp. 15761589, June 2014.

[215] Y.-R. Chien, "Iterative channel estimation and impulsive noise mitigation algorithm for OFDM-based receivers with application to powerline communications," IEEE Trans. Power Del., vol. 30, no. 6, pp. 2435-2442, June 2015.

[216] Y.-R. Chien, J.-W. Chen, and S. S.-D. Xu, "A multilayer perceptronbased impulsive noise detector with application to power-line-based sensor networks," IEEE Access, vol. 6, pp. 21 778-21 787, June 2018.

[217] P. Pagani, R. Hashmat, A. Schwager, D. Schneider, and W. Bäschlin, "European MIMO PLC field measurements: Noise analysis," in Proc IEEE Int. Symp. Power Line Commun. Appl. (IEEE ISPLC 2012), Beijing, China, March 2012, pp. 310-315.

[218] M. Elgenedy, M. Sayed, and N. Al-Dhahir, "A frequency-shift-filtering approach to cyclostationary noise modeling in MIMO NB-PLC," in Proc. IEEE Global Conf. Signal Inf. Process. (GlobalSIP 2016), Washington, DC, USA, Dec 2016, pp. 881-885.

[219] T. N. Vo, K. Amis, T. Chonavel, P. Siohan, and P. Pagani, "Influence of interference in MIMO power line communication systems," in Proc. IEEE Int. Symp. Power Line Commu. Appl. (IEEE ISPLC 2014), Glasgow, UK, March 2014, pp. 255-260.

[220] R. Pighi and R. Raheli, "On multicarrier signal transmission for highvoltage power lines," in Proc. IEEE Int. Symp. Power Line Commun. Appl. (ISPLC 2005), Vancouver, BC, Canada, April 2005, pp. 32-36.

[221] W. De Villiers, J. H. Cloete, L. M. Wedepohl, and A. Burger, "Realtime sag monitoring system for high-voltage overhead transmission lines based on power-line carrier signal behavior," IEEE Trans. Power Del., vol. 23, no. 1, pp. 389-395, Jan. 2007.

[222] Y. Liu and X. Chen, "Design of traffic lights controlling system based on PLC and configuration technology," in Proc. IEEE Int. Conf. Multimedia Inf. Netw. Security (MINES 2009), Nov. 2009, pp. 561-563.

[223] F. Basile, P. Chiacchio, and D. Gerbasio, "On the implementation of industrial automation systems based on PLC," IEEE Trans. on Automa. Sci. Eng., vol. 10, no. 4, pp. 990-1003, April 2013.

[224] Y. Qian, L. Shi, J. Li, X. Zhou, F. Shu, and J. Wang, "An edgecomputing paradigm for Internet of Things over power line communication networks," IEEE Netw., pp. 1-8, 2020. 
[225] A. M. Tonello, N. A. Letizia, D. Righini, and F. Marcuzzi, "Machine learning tips and tricks for power line communications," IEEE Access, vol. 7, pp. 82 434-82 452, 2019.

[226] S. Dörner, S. Cammerer, J. Hoydis, and S. ten Brink, "Deep learning based communication over the air," IEEE J. Sel. Topics Signal Process., vol. 12, no. 1, pp. 132-143, Jan. 2018.

[227] I. Mann, S. McLaughlin, W. Henkel, R. Kirkby, and T. Kessler, "Impulse generation with appropriate amplitude, length, inter-arrival, and spectral characteristics," IEEE J. Sel. Areas Commun., vol. 20, no. 5, pp. 901-912, May 2002.

[228] B. Drooghaag, J. Maes, M. El Fani, and V. Moeyaert, "Exploring field noise on G.fast frequencies," in Proc. IEEE Global Telecomm. Conf. (GLOBECOM 2017). Singapore, Singapore: IEEE, Dec. 2017, pp. $1-6$.

[229] T. Bai, C. Xu, R. Zhang, A. F. Al Rawi, and L. Hanzo, "Performance of HARQ-assisted OFDM systems contaminated by impulsive noise: Finite-length LDPC code analysis," IEEE Access, vol. 7, pp. 14112 $14123,2019$.

[230] W. Coomans, R. B. Moraes, K. Hooghe, A. Duque, J. Galaro, M. Timmers, A. J. van Wijngaarden, M. Guenach, and J. Maes, "XG-fast: the 5th generation broadband," IEEE Communications Magazine, vol. 53, no. 12 , pp. 83-88, Dec. 2015.

[231] J. M. Cioffi, K. J. Kerpez, C. S. Hwang, and I. Kanellakopoulos, “Terabit DSLs," IEEE Communications Magazine, vol. 56, no. 11, pp. 152-159, Nov. 2018

[232] X. Kuai, H. Sun, S. Zhou, and E. Cheng, "Impulsive noise mitigation in underwater acoustic OFDM systems," IEEE Transactions on Vehicular Technology, vol. 65, no. 10, pp. 8190-8202, Oct. 2016.

[233] A. Mahmood, M. Chitre, and H. Vishnu, "Locally optimal inspired detection in snapping shrimp noise," IEEE J. Ocean. Eng., vol. 42, no. 4, pp. 1049-1062, April 2017.

[234] D. C. Bertilone and D. S. Killeen, "Statistics of biological noise and performance of generalized energy detectors for passive detection," IEEE J. Ocean. Eng., vol. 26, no. 2, pp. 285-294, April 2001.

[235] M. A. Chitre, J. R. Potter, and S.-H. Ong, "Optimal and near-optimal signal detection in snapping shrimp dominated ambient noise," IEEE J. Ocean. Eng., vol. 31, no. 2, pp. 497-503, Apr. 2006.

[236] P. Chen, Y. Rong, S. Nordholm, Z. He, and A. J. Duncan, "Joint channel estimation and impulsive noise mitigation in underwater acoustic OFDM communication systems," IEEE Trans. Wireless Commun., vol. 16, no. 9, pp. 6165-6178, Sept 2017.

[237] M. Cheffena, "Propagation channel characteristics of industrial wireless sensor networks [wireless corner]," IEEE Antennas and Propagation Magazine, vol. 58, no. 1, pp. 66-73, Feb 2016.

[238] S. Liu, F. Yang, W. Ding, and J. Song, "Double kill: Compressivesensing-based narrow-band interference and impulsive noise mitigation for vehicular communications," IEEE Transactions on Vehicular Technology, vol. 65, no. 7, pp. 5099-5109, July 2015.

[239] P. Torio and M. G. Sanchez, "Mitigation of impulsive noise in digital video broadcasting terrestrial using orthogonal polarization reception," IEEE Transactions on Consumer Electronics, vol. 55, no. 4, pp. 17981804, Sept. 2009.

[240] M. S. Alam, B. Selim, G. Kaddoum, and B. L. Agba, "Mitigation techniques for impulsive noise with memory modeled by a two state Markov-Gaussian process," IEEE Systems Journal, Early access.

[241] N. Shlezinger and R. Dabora, "On the capacity of narrowband PLC channels," IEEE Trans. Commun., vol. 63, no. 4, pp. 1191-1201, April 2015 .

\section{GLOSSARY}

$\begin{array}{ll}\text { AC } & \text { Alternating Current } \\ \text { ACK } & \text { Acknowledgment } \\ \text { ARMA } & \text { Auto-Regressive Moving Average } \\ \text { ARQ } & \text { Automatic-Repeat-and-reQuest } \\ \text { AWGN } & \text { Addictive White Gaussian Noise } \\ \text { BB } & \text { Broad-Band } \\ \text { BER } & \text { Bit Error Rate } \\ \text { BGM } & \text { Bernoulli-Gaussian Model } \\ \text { CDF } & \text { Cumulative Distribution Function } \\ \text { CE } & \text { Channel Estimation } \\ \text { CIS } & \text { Compressed Impairment Sensing } \\ \text { CS } & \text { Compressed Sensing }\end{array}$

\begin{tabular}{|c|c|}
\hline DFT & Discrete Fourier Transform \\
\hline DL & Deep Learning \\
\hline EM & Expectation Maximization \\
\hline FD & Frequency Domain \\
\hline FEC & Forward Error Correction \\
\hline FIR & Finite Impulse Response \\
\hline FSK & Frequency Shift Keying \\
\hline GMM & Gaussian Mixture Model \\
\hline HARQ & Hybrid-Automatic-Repeat-and-reQuest \\
\hline HMM & Hidden Markov Model \\
\hline HSMM & Hidden Semi-Markov Model \\
\hline IAT & Inter-Arrival Time \\
\hline IBA & Iterative Bayesian Algorithm \\
\hline IDFFT & Interleaved-Double-FFT \\
\hline IDFT & Inverse Discrete Fourier Transform \\
\hline JEVA & Joint Erasure Marking and Viterbi Algorithm \\
\hline JELVA & $\begin{array}{l}\text { Joint Erasure Marking and List Viterbi } \\
\text { Algorithm }\end{array}$ \\
\hline LDPC & Low-Density Parity-Check \\
\hline LLR & Log-Likelihood Ratio \\
\hline LPTV & Linearly Periodic Time-Varying \\
\hline LTI & Linearly Time-Invariant \\
\hline MAP & Maximum a Posteriori \\
\hline $\mathrm{MC}$ & Multi-Carrier \\
\hline MCA & Middleton's Class A \\
\hline MIMO & Multiple-Input Multiple-Output \\
\hline ML & Machine Learning \\
\hline MMSE & Minimum Mean Square Error \\
\hline NACK & Non-Acknowledgment \\
\hline NB & Narrow-Band \\
\hline OFDM & Orthogonal Frequency Division Multiplexing \\
\hline PA-SAMP & Priori Aided Sparsity Adaptive Matching Pursuit \\
\hline PAPR & Peak-to-Average-Power Ratio \\
\hline PDF & Probability Density Function \\
\hline PLC & Power-Line Communications \\
\hline PSD & Power Spectral Density \\
\hline PSK & Phase Shift Keying \\
\hline QAM & Quadrature Amplitude Modulation \\
\hline QoS & Quality of Service \\
\hline $\mathrm{SC}$ & Single-Carrier \\
\hline SISO & Single-Input Single-Output \\
\hline SNR & Signal-to-Noise Ratio \\
\hline $\mathrm{TD}$ & Time Domain \\
\hline TDI & Time-Domain Interleaver \\
\hline TDI-OT & $\begin{array}{l}\text { Time-Domain Interleaver with Additional } \\
\text { Orthogonal Transform }\end{array}$ \\
\hline UNB & Ultra-Narrowband \\
\hline$\alpha S$ & Alpha-Stable \\
\hline
\end{tabular}




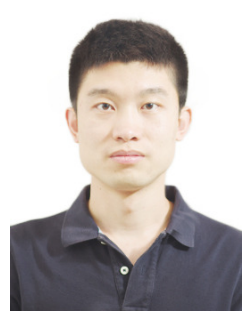

Tong Bai (S'15-M'19) received the B.Sc. degree in telecommunications from Northwestern Polytechnical University, Xi'an, China, in 2013, and the M.Sc. and Ph.D. degrees in communications and signal processing from the University of Southampton, Southampton, U.K., in 2014 and 2019, respectively. Since 2019, he has been a Postdoctoral Researcher with Queen Mary University of London, London, U.K. His research interests include the performance analysis, transceiver design, and utility optimization for power-line and wireless communications as well as for Internet of Things. Dr. Bai was a recipient of 2019 Chinese Government Award for Outstanding Self-Financed Student Abroad.

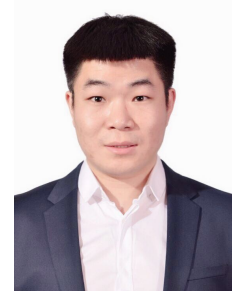

Hongming Zhang (S'15-M'17) received the B.Eng. degree (Hons.) in telecommunications from the Nanjing University of Aeronautics and Astronautics (NUAA) and the City University of London, in 2011 , the M.Sc. and Ph.D. degrees in wireless communications from the University of Southampton in 2012 and 2017, respectively. From 2017-2018, he was with the Department of Electrical Engineering, Columbia University, New York, NY, USA. He is currently an Assistant Professor with the School of Information and Communication Engineering, Beijing University of Posts and Telecommunications, Beijing, China. His research interests include wireless communications, heterogeneous network performance analysis and optimization, and machine learning.

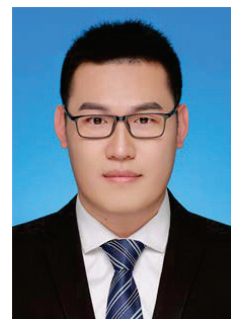

Jingjing Wang (S'14-M'19) received his B.S. degree in Electronic Information Engineering from Dalian University of Technology, Liaoning, China in 2014 and the Ph.D. degree in Information and Communication Engineering from Tsinghua University, Beijing, China in 2019, both with the highest honors. From 2017 to 2018, he visited the Next Generation Wireless Group chaired by Prof. Lajos Hanzo, University of Southampton, UK. Dr. Wang is currently a postdoc researcher at Department of Electronic Engineering, Tsinghua University. His research interests include resource allocation and network association, learning theory aided modeling, analysis and signal processing, as well as information diffusion theory for mobile wireless networks. Dr. Wang was a recipient of the Best Journal Paper Award of IEEE ComSoc Technical Committee on Green Communications \& Computing in 2018, the Best Paper Award of IEEE ICC and IWCMC in 2019.

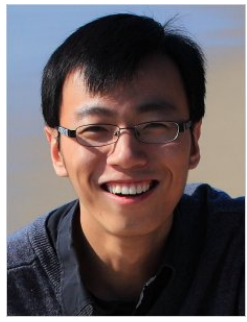

Chao Xu (S'09-M'14) received a B.Eng. degree from Beijing University of Posts and Telecommunications, China, and a BSc(Eng) with First Class Honours from Queen Mary, University of London, UK, through a Sino-UK joint degree program in 2008, both in Telecommunications Enginneering with Management. He obtained a MSc degree with distinction in Radio Frequency Communication Systems and a Ph.D. degree in Wireless Communications from the University of Southampton, UK in 2009 and 2015, respectively. He is currently a postdoctoral researcher working at Southampton Wireless Group, University of Southampton, UK. His research interests include reduced-complexity MIMO design, noncoherent detection, extrinsic-information-transfer-chart-aided turbo detection, and cooperative communications. He was awarded the Best M.Sc. Student in Broadband and Mobile Communication Networks by the IEEE Communications Society (United Kingdom and Republic of Ireland Chapter) in 2009. He also received 2012 Chinese Government Award for Outstanding Self-Financed Student Abroad.

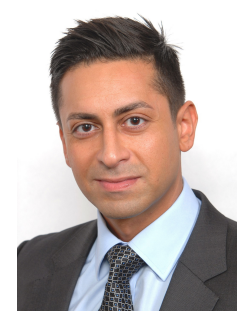

Maged Elkashlan received the Ph.D. degree in Electrical Engineering from the University of British Columbia, Canada, 2006. From 2007 to 2011, he was with the Commonwealth Scientific and Industrial Research Organization (CSIRO), Australia. During this time, he held visiting appointments at University of New South Wales and University of Technology Sydney. In 2011, he joined the School of Electronic Engineering and Computer Science at Queen Mary University of London, UK. His research interests fall into the broad areas of communication theory and statistical signal processing.

Dr. Elkashlan currently serves as Editor of IEEE TRANSACTIONS ON WirELESS COMMUNICATIONS and IEEE TRANSACTIONS ON VEHICULAR Technology. He received the Best Paper Awards at the IEEE International Conference on Communications (ICC) in 2016 and 2014, the International Conference on Communications and Networking in China (CHINACOM) in 2014, and the IEEE Vehicular Technology Conference (VTC-Spring) in 2013.

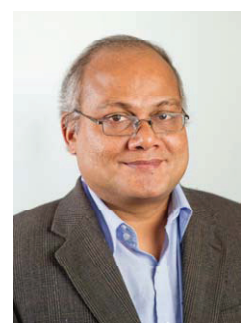

Arumugam Nallanathan (S'97-M'00-SM'05-F'17) is Professor of Wireless Communications and Head of the Communication Systems Research (CSR) group in the School of Electronic Engineering and Computer Science at Queen Mary University of London since September 2017. He was with the Department of Informatics at King's College London from December 2007 to August 2017, where he was Professor of Wireless Communications from April 2013 to August 2017 and a Visiting Professor from September 2017. He was an Assistant Professor in the Department of Electrical and Computer Engineering, National University of Singapore from August 2000 to December 2007. His research interests include Artificial Intelligence for Wireless Systems, Beyond 5G Wireless Networks, Internet of Things (IoT) and Molecular Communications. He published nearly 500 technical papers in scientific journals and international conferences. He is a co-recipient of the Best Paper Awards presented at the IEEE International Conference on Communications 2016 (ICC'2016), IEEE Global Communications Conference 2017 (GLOBECOM'2017) and IEEE Vehicular Technology Conference 2018 (VTC'2018). He is an IEEE Distinguished Lecturer. He has been selected as a Web of Science Highly Cited Researcher in 2016 and an AI 2000 Internet of Things Most Influential Scholar in 2020.

$\mathrm{He}$ is an Editor-at-Large for IEEE Transactions on Communications and Senior Editor for IEEE Wireless Communications Letters. He was an Editor for IEEE Transactions on Wireless Communications (2006-2011), IEEE Transactions on Vehicular Technology (2006-2017) and IEEE Signal Processing Letters. He served as the Chair for the Signal Processing and Communication Electronics Technical Committee of IEEE Communications Society and Technical Program Chair and member of Technical Program Committees in numerous IEEE conferences. He received the IEEE Communications Society SPCE outstanding service award 2012 and IEEE Communications Society RCC outstanding service award 2014.

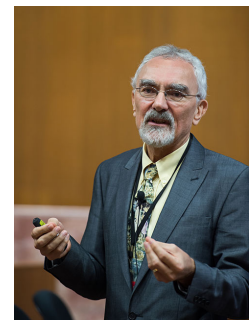

Lajos Hanzo (https://www-mobile.ecs.soton.ac.uk) (FIEEE'04, Fellow of the Royal Academy of Engineering $\mathrm{F}(\mathrm{REng})$, of the IET and of EURASIP), received his Master degree and Doctorate in 1976 and 1983, respectively from the Technical University (TU) of Budapest. He was also awarded the Doctor of Sciences (DSc) degree by the University of Southampton (2004) and Honorary Doctorates by the TU of Budapest (2009) and by the University of Edinburgh (2015). He is a Foreign Member of the Hungarian Academy of Sciences and a former Editor-in-Chief of the IEEE Press. He has served several terms as Governor of both IEEE ComSoc and of VTS. He has published 1900+ contributions at IEEE Xplore, 19 Wiley-IEEE Press books and has helped the fast-track career of $123 \mathrm{PhD}$ students. Over 40 of them are Professors at various stages of their careers in academia and many of them are leading scientists in the wireless industry. 